\title{
Pilzstudien in Finnland
}

\author{
Otto von Schulmann \\ Einleitung
}

P. A. K a r s te n, Finnlands grösster Pilzforscher, starb vor 38 Jahren und seine Hauptwerke erschienen 1879, 1882 und 1889 mit Ergänzungen bis 1897. Seitdem hat die Pilzkunde reissende Fortschritte gemacht. Neue Untersuchungsmethoden wurden eingeführt, die auf den grossen Fries zurückgehende Systematik musste tief greifenden Modifikationen unterworfen und zahlreiche neue Arten konnten festgestellt werden. Entsprechend sind die Arbeiten unseres Altmeisters heute zu einem nicht geringen Teil als veraltet anzusehen, und die Pilzfreunde Finnlands warten heute nicht ohne eine gewisse Ungeduld auf einen neuen Karsten, der die Fähigkeit hätte, ausgehend von den hervorragenden Arbeiten des Vorgängers, diejenigen Veränderungen und Verbesserungen vorzunehmen, die mittlerweile unvermeidlich geworden sind.

Die Zeiten des Mykologen alten Schlages sind für immer dahin. Der zukünftige Karsten wird nicht wie Fries und unser Altmeister das Material selbst sammeln, sichten und verarbeiten. Im Besitze eines umfassenden allgemeinen Wissens auf dem Gebiete der höheren Pilze wird er das in den letzten Jahrzehnten in der Hauptsache von Anderen gesammelte Material prüfen, das Unbrauchbare ausscheiden und die genügend belegten Funde in eine moderne wissenschaftliche Form bringen müssen. Seine Arbeit wird also Vorarbeiten anderer Pilzkenner zur unbedingten Voraussetzung haben, die sich dem Studium der höheren Pilze bald eines bestimmten geographischen Gebietes, bald einer oder verschiedener Pilzgattungen widmeten.

Ich bin Jurist und Nationalökonom und nicht Naturwissenschaftler. Entsprechend erhebe ich nicht den Anspruch ein neuer Karsten zu sein. Ich zähle mich zu seinen
Vorarbeitern, die sich die Feststellung der Flora gewisser geographischer Bezirke zur Aufgabe gemacht haben. Dazu befähigen mich die Erfahrungen, die ich im Laufe vieler Jahre in Finnland und anderswo gesammelt habe. Der Anfang meiner Studien liegt weit zurück, indem ich mich schon in den 20-er Jahren in Estland mit den höheren Pilzen beschäftigte. Meine Studien in Finnland hatten 1941-1946 mehr sporadischen Charakter. 1947 war ein Ubergangsjahr, indem ich mich damals in der Gegend von Bromarv, bewaffnet mit Mikroskop und einer Reihe mehr oder weniger populärer, aber zum Bestimmen durchaus brauchbarer Werke, den Pilzen widmete. Die Jahre 1948-1953 waren solche systematischer Untersuchungen.

Anfangs standen mir nur folgende Werke zur Verfügung:

- E. In g e ls tr ö m: Svampflora 1940.

- P. A. K a r s t e n: Rysslands, Finlands och den Skandinaviska halföns hattsvampar. 1879-1882.

- P. A. K a r s t e n: Kritisk öfversigt af Finlands Basidsvampar 1889 nebst 3 späteren Ergänzungen.

- P. A. K a r s t e n: Finlands Basidsvampar. 1899.

- J. E. L a n g e: Flora Agaricina Danica. 1935-1940.

- E. M i ch a e 1: Führer für Pilzfreunde. Auflage 1927. 3 Bände.

- E. M i ch a e l: Führer für Pilzfreunde. Auflage 1939. Erster Band.

- A. Ricken: Vademecum für Pilzfreunde. 1920. 
Gelegentlich konnte mit Nutzen Einblick genommen werden in:

- A. Andersson: Buksvampar. (In B. Ursing s: Svenska växter - Kryptogamer. 1949).

- E. G r a m b e r g: Pilze der Heimat 1939. Zwei Bände.

- Krok och A l m quis t: Svensk Flora. Kryptogamerna. 7 Auflage (Polyporeae). Verschiedene Bildwerke und Pilztafeln. Specialliteratur über die Pilze des Balticums, insbes. Estlands.

$\mathrm{Zu}$ ihnen gesellten sich später:

- H. Bourdot et A. Galzin: Hétérobasidiés-Homobasidiés gymnocarpes. 1927.

- J. F avre: Les champignons collybioïdes des cônes des essences résineuses. Schweiz. Zeitschrift für Pilzkunde 1939.

- P. Konrad: et A. Maublanc: Les Agricales 1948-1952.

- M. M os e r: Blätter- und Bauchpilze 1953.

M. M o s e r: Cortinariusstudien. I Phlegmacium. Sydowia 1951-1953.

- A. A. Pearson: Notes on the Boleti. The Naturalist 1946.

- A. A. P e a r s o n: The genus Russula. The Naturalist 1948.

- A. A. P e a r s o n: The genus Lactarius. The Naturalist 1950.

- R. Tuomikoski: Suomen rouskut. Luonnon Tutkija 1952.

- R. Tu omikoski: Die LactariusArten Finnlands. Karstenia 1953.

Infolge fehlender Literatur sind meine Kenntnisse der Ascomycetes sehr lückenhaft.

Die mikroskopischen Untersuchungen bezogen sich zuerst nur auf die Sporenformen und -grössen. 1952 und 1953 wurde viel Zeit den Cystiden und anderen mikroskopischen Merkmalen, so der »Ornamentation» der Russula-Sporen, gewidmet. In den gleichen Jahren wurden auch einige Chemikalien zur Feststellung der chemischen Reaktionen verwandt. In Parallele zu der Vermehrung der Literatur und der Ver- feinerung der Untersuchungsmethoden wuchsen natürlich die Erfahrung und die Sicherheit der Bestimmungen. Letztere ist für die Jahre 1952 und 1953 ganz erheblich grösser als bis dahin. Völlige Sicherheit der Bestimmungen wurde stets erstrebt und häufig nicht erreicht. Wer sich Unfehlbarkeit zuschreibt sollte seine Finger von der Mykologie tunlichst ferne halten.

Auf die Herstellung von Exsikkaten musste anfangs verzichtet werden. Die Pensionate, die nolens volens benutzt werden mussten, sind dazu meistens ungeeignet. Ich half mir damals damit, dass ich schwer zu bestimmende Arten verschiedenen erfahrenen Beobachtern, vor allen Dir. W. N y b e r g, per Post zur Begutachtung schickte. Mancher meiner Pilze hat so schliesslich seinen Weg in dieses oder jenes Herbarium gefunden. Vieles ist auf dem Wege verdorben. Erst 1952 und 1953 konnte unter nahezu idealen Bedingungen getrocknet werden. Alle Exsikkate, auch die vom J. 1951, sind von mir im Bot. Institut der Universität Helsinki deponiert worden, wo sie jederzeit in Augenschein genommen werden können. Sie sind im Allgemeinen befriedigender Qualität, doch befinden sich unter ihnen auch schlechte und solche nur von Pilzteilen, z.B. Reste untersuchter Unica. Der Grund ist einfach der, dass die Exsikkate nicht für ein Herbarium gesammelt wurden. Sie haben eine andere Zweckbestimmung, indem sie nämlich eine Nachprüfung der Bestimmungen ermöglichen sollen. Bekanntlich vermögen mikroskopische und chemische Untersuchungen von Exsikkatmaterialien häufig sehr wertvolle Aufschlüsse zu vermitteln, vorausgesetzt dass das Material gut aufbewahrt wurde.

Die Funde sind nach den Suchstellen geordnet. Innerhalb der Suchstellen erfolgte die Gruppierung der Gattungen, Familien und Arten im Falle der Agaricales und Gastromycetes nach M. M o s e r. Im Falle der Cantharellales und der Ascomycetes geschah die Gruppierung nach $\mathrm{R} \mathrm{icke} \mathrm{n}$ und im Falle der übrigen Basidiomycetes nach $\mathrm{B}$ o u rdot et Galzin.

Die Masse der Hüte sind in $\mathrm{cm}$. angegeben. Bei den Füssen bedeutet $10 \times 1510$ $\mathrm{cm}$ lang und $15 \mathrm{~mm}$ breit. Die mikroskopischen Masse sind in Mikromillimetern, Farbenskala bei Russula nach $\mathrm{P}$ e a r s o von $\mathrm{A}$ (weiss) bis $\mathrm{H}$ (dottergelb), Ornamenta- 
tion der Sporen bei Russula nach $\mathrm{P}$ e a rso n (11 Klassen). Das Datum zeigt den ersten Fund des Jahres der betreffenden Suchstelle.

\section{Abkürzungen:}

$\begin{array}{llrl}\text { Cyst. } & \text { Cystiden } & & \\ \text { F. } & \text { Fuss } & \text { P. } & \text { Poren } \\ \text { Fl. } & \text { Fleisch } & \text { R. } & \text { Röhrchen } \\ \text { H. } & \text { Hut } & \text { Sp. } & \text { Sporen } \\ \text { L. } & \text { Lamellen } & \text { St. } & \text { Stacheln }\end{array}$

Die allmähliche Form- und Farbenveränderung wird gewöhnlich durch einfachen Bindestrich wiedergegeben, z.B. bedeutet »glockig-gebuckelt-flach», dass der Pilz zuerst glockig, dann gebuckelt und schliesslich flach ist.

Ich schliesse mit einem Dank an Bankdirektor W. Nyberg, die Professoren V. $\mathrm{Kujala}$ und R. Tuomikoski und Magister N. Malmström, die mich häufig mit ihrem Rat unterstützten.

\section{I. Ålands Sommerpilze}

Aland wird häufig unter Hinweis auf die grosse Waldfläche als ein Pilzland par excellence bezeichnet. Dies ist nicht ganz zutreffend, da ein grosser Teil der Wälder eigentlich Ödland darstellen. Der auf diesem wachsende Kiefernbestand kann geradezu als eine Art natürlichen Bannwaldes bezeichnet werden, da er infolge höchst geringer Holzqualität und schlechter Verbindungen für eine wirtschaftliche Verwertung vorläufig nicht in Frage kommt. Hier kann man stundenlang herumschweifen, ohne auch nur auf einen einzigen interessanteren Pilz zu Gesicht zu bekommen. Sollte man daraus den Schluss ziehen, dass Åland dem Mykologen nichts zu bieten hat? Nichts wäre falscher als dies. Es gibt hier auch ergiebige Pilzgebiete, wenn diese auch nicht sonderlich zahlreich und gross sind. Der Reiz der Pilzflora Ålands für den finnländischen Pilzforscher besteht nicht nur darin, dass sie kaum systematisch untersucht wor- den ist, sondern vor allem in ihrem deutlichen Übergangscharakter. Es ist eine finnländische Flora, jedoch mit deutlichen Anklängen an Schweden, Westestland und die Insel Ösel. Dies gilt vor allem für die westlichen und südlichen Teile des Landes.

Ich hatte Gelegenheit die Pilzflora Ålands in den Sommern 1948-1950 zu studieren und zwar verbrachte ich:

- auf Eckerö (E.) in Weståland die Zeit vom 1.6. bis zum 30.8. 1948.

- auf Finström, Hjortö (H.) in Mittelåland die Zeit vom 4.6. bis zum 6.8. 1949.

- auf Lemland, Lemböte (L.) in Südåland die Zeit vom 14.6 bis zum 27.8. 1950.

Eingeschaltet wurden eine Reihe von Besuchen auf einigen Inseln des Nordwestens und Südens, in Geta, Saltvik, Kastelholm, Sund, Bomarsund, Jomala und in der Gegend von Mariehamn.

Über die Studien auf Eckerö 1954 siehe den Nachtrag.

\section{BASIDIOMYCETES.}

Suillus luteus L. - E. L.

S. flavidus Fr. - L. 15.8.

S. granulatus L. - E. H. L.

$S$. bovinus L. - E.

S. variegatus Sow. - E. H. L.

S. piperatus Bull. - E. H. L.

Leccinum rufum Schff. - E. L. - Sammelart. S. Kap. 5.

L. scabrum Bull. - E. H. L. - Sammelart. S. Kap. 5.

Boletus luridus Fr. - E. H. und bei Mariehamn. -

Dieser auffallende Pilz ist auf dem Festlande

Finnlands nahezu unbekannt. Er ist auf den mit Laubhölzern bewachsenen Wiesen Alands nicht gerade selten. In Estland ist er gemein.

B. edulis Bull. - E. H. L.

Tylopilus felleus Bull. - E. L. 6.8.
Xerocomus badius Fr. - E.

$X$. subtomentosus L. - E. L.

Paxillus involutus Batsch - E. L.

$P$. atrotomentosus Batsch - E. H. L.

Hygrophoropsis aurantiaca Wulf. - E.

Gomphidius glutinosus Schff. - E. L.

G. roseus Fr.. - L. 5.8 .

G. viscidus L. (rutilus Schff.) - E. H. L.

Hygrophorus Karstenii Sacc. et Cub. (bicolor Karst.) E. - Sieht Camarophyllus pratensis sehr ähnlich. Blass mit orange L. Sp. $8-105-6$. Exs.: E. 21.9.54. 
H. olivaceoalbus Fr. ${ }^{\text {" }}-\mathrm{E}$.

H. erubescens Fr. - E.

H. pudorinus Fr. - 'E. (Torp) 8.8. L. 23.8. - Siehe Kap. 4.

H. agathosmus Fr. - E. L. 27.7.

H. caprinus Scop. - E. 15.8 .

Camarophyllus pratensis Pers.. - E.

Hygrocybe tristis Pers. (nigrescens Quel. var.) - L. 26.7. - Siehe Kap. 4.

H. conica Scop. - E.

H. Langei Kühn. (obrussea Rick., constans Lange, amoena R. Sch.) - E. Juli, Aug. H. 5.8. L. 19.7. - Siehe Nachtrag c).

H. miniata Fr. - E.

Asterophora lycoperdoides Bull. (Nyctalis asterophora Fr.) - E.

A. parasitica Bull. - L. 14.8. - Glatte Ghlamydosporen, $12-15 \times 9$.

Lyophyllum conglobatum Vitt. (aggregatum K. et M.) L. 14.8.

L. decastes Fr. (aggregatum Schff.) - L. 14.8. - Siehe Kap. 5.

Calocybe Georgii Clus. (Tricholoma gambosum Fr.) E. H. L. - Sehr selten auf dem Festlande Finnlands. Überall a. Åland.

Clitocybe geotropa Bull, var. subinvoluta - L. 26.7. Siehe Kap. 5.

C. Alexandri Gill. (Paxillus extenuatus Fr.) - L. 4.8. - Siehe Kap. 4.

C. odora Bull. - E.

C. clavipes Pers. - E.

C. gilva Pers. - L. 10.7. - Siehe Kap. 5 .

C. squamulosa Pers. - L. 8.7 .

C. infundibuliformis Schff. - E. H. L.

C. sinopica Fr. - $\mathrm{H}$.

C. phyllophila Fr. - E. Juli und Aug.

C. rivulosa Pers. - L. 6.7.

C. obsoleta Batsch - L. 31.7. - Siehe Kap. 4.

C. fragrans Sow. - E.

C. suaveolens Schum. - H. - Im Gegensatz zu C. fragrans dunkel genabelt. Var.?.

Laccaria la lata Scop. var. proxima - L. 2.8.

L. laccata Scop. var. rosella - E. H. L.

L. laccata Scop. var. rufocarnea - E.

L. echinospora Speg. (tortilis (Bolt.) Fr. ss. Bres.) L. 7.8. - H. bis 1 breit. F. $1 \times 1$. Sp. rund, $11-12$.

Tricholomopsis rutilans Schff. - E. .L.

Collybia fuscopurpurea Pers. - L. 27.7. Sehr selten. - H. bis 4.5 breit, rotbraun. F. dunkel purpurbraun, unten filzig. Wurzelige Basis. L. rotbraun. Sp. apfelkern- bis lanzettförmig, $8 \times 3$. Dir. Ny berg zugesandt.

C. dryophila Bull. - E. H. L.

C. asema Fr. - E. - Siehe Kap. 4.

Omphalina umbellifera $\mathrm{L} .-\mathrm{H}$.

O. umbellifera L. f. albida - L. 7.7.

O. abiegna Bk. et $\mathrm{Br}$. (bibula Quel., umbellifera L. var. citrina Quel.) - H. 9.6. - H. bis 2 breit, citr. gelb. F. und L. gleichfarbig. L. entfernt. Sp. $8 \times 5$, eiförmig. Auf einem Nadelholzstumpf. Armillariella mellea Vahl - E.

Tricholoma focale Fr. - E. 28.7. - H. 6-7 cm. breit, rotbraun. F. mit halsbandartigem schiefsitzendem Ring. Sp. $4 \times 3$. Mehlgeruch und Mehlgeschmack. Sehr selten. Ich fand nur zwei Ex. im Nadelwalde. Karstens Pilz nicht identisch. Dir. N y b e r g zugeschickt.

T. flavobrunneum Fr. - E. I. 6.8 .
T. imbricatum Fr. - E.

T. sejunctum Sow. - E. 12.8. L. 19.8.

T. quinquepartitum Fr. - E. (Torp) 24.8. - H. gelb, etwas klebrig. F. weiss bis 10 lang. L. weiss und breit. Sp. $4-5.5 \times 3.5-4.5$ elliptisch. Mehlgeruch und Mehlgeschmack. Sieht Tr. sejunctum sehr ähnlich. Selten.

T. equestre L. $-\mathrm{E}$.

T. inamoenum Fr. - E.

T. saponaceum Fr. - E. L.

T. saponaceum Fr. var. ardosiacum Bres. - E. L.

T. virgatum $\mathrm{Fr} .-\mathrm{H}$. L. 15.8.

T. terreum Schff. - E.

T. myomyces Lange (scalpturatum Fr.?) - H. Kleiner als T. terreum. Im Gegensatz zu $T$. terreum mit bärtig-wolligem Rand. Spuren einer Cortina. Sp. $5.5 \times 3.5-3.75$.

Aspropaxillus giganteus Fr. - E. (Torp). Ende Aug. L. 23.8. - Grosser, blasser, trichterförmiger Grasparasit. Sp. 6-7 $\times 4-5$. Auf. dem Festlande Finnlands sehr selten.

Melanoleuca polioleuca Fr. - E. 24.8. - Wie $M$. melaleuca Pers. Mit stark bereiftem H.

M. strictipes Karst. (pubifolium Romell). - E. H. L.

Lentinellus omphalodes Fr. - L. 30.7. - H. etwa 3 breit, von bleicher Farbe, mit dunkleren Schuppen. F. bis $4 \times 6$, fas. gestreift. Sp. $5.5 \times 4$, eiförmig bis oval. Auf dem Erdboden, vermutlich auf verfaulten Holzresten. Unsichere Bestimmung.

Panus conchatus Fr. - E. H.

Lentinus lepideus Fr. - E. H. L.

Mucidula radicata Relh. - Lemström Kanal 27.8.50.

Micromphale perforans Hoffm. - H. L.

Pseudohiatula esculenta Wulf. - E. H. L. - Sammelart. Die Auftejlung derselben war mit, als ich auf $\AA$ land war, noch nicht bekannt.

Marasmius scorodonius Fr. - E. H.

$M$. oreades Bolt. - E. H. L.

$M$. graminum Lib. ss. Karst. nec. Langei - E. Hut 2-3 $\mathrm{mm}$ breit, blassrötlich bis bräunlich mit dunklerem Wärzchen in der Mitte. L. 6-8 Stück, entfernt, gleichlang, mit freiem Halsring den Fuss umschliessend. Sp. $6.5-7.5 \times 3.5$. Auf Gräsern und Blättern.

M. rotula Scop. - E. - H. weiss, $10-15 \mathrm{~m} / \mathrm{m}$ breit. L. zu einem Halsband verbunden, Sp. $9 \times 4$. Auf Laubholzästen.

$M$. Wettsteinii Sacc. et Sydow (tenerrimus Wettst., Nybergii OvS.) - H. 3.8. L. 28.7. - Siehe Kap. 5.

M. androsaceus L. - E. L. 28.7.

Xerophalina campanella Batsch - E.

Mycena vitrea Fr. ss. Rick. (sepia Lange.) - L. 7.7. H. bis 2 breit, konisch-glockig oder parabolisch, schwarzbraun, $3 / 4$ gerieft. F. gleichfarbig, etwas blasser, oben weisslich, $6 \times 1$. L. grau mit hellerem Rand. Sp. 8-9 4 4.5-5, elliptisch.

M. galericulata Scop. - E. L.

$M$. pura Pers. - E. H.

M. polygramma Bull. - H. 14.6. - H. bis 3 breit, braungrau, runzelig-gerieft. F. gefurcht-gerillt, lang, bläulichgrau, seidigglänzend, sehr hart, mit spindelförmiger langer Fortsetzung. Sp. $8-10 \times 5-6$. A. Stämmen.

M. delectabilis Peck (Omphalia gracillima Weinm.) E. - Ganz weiss. H. bis 1 breit, durchscheinend gerieft. F. bis 2 hoch, mit flockigstrahliger Basis dem Substrat (meist Holz) aufsitzend. L. dünn, breit, nur wenige. Sp. $6-7 \times 3-4$, ei- bis kernförmig. 
Clitopilus prunulus Scop. - E. H.

Rhodophyllus clypeatus L. - H. 8.6. L. 17.7.

$R$. nidorosus $\mathrm{Fr} .-\mathrm{E}$.

R. rhodopolius Fr. - E. L. 26.7. - Siehe Nachtrag c).

$R$. staurosporus Bres. - E. $\mathrm{H}$

$R$. sericeus Bull. - E. L. 30.7 .

R. griseorubellus Lasch ss. Karst. - L. 26.8. Siehe Kap. 4.

R. asprellus Fr. - E. L. 2.7.

$R$. serrulatus Fr. - E.

$R$. griseocyaneus Fr. - L. 30.6

$R$. lampropus Fr. - E. L. 1.8.

R. minutus Karst. - L. 20.7. - Siehe Kap. 4.

Pluteus cervinus Schff. - E. H. L.

P. umbrosus (Pers.) Fr. ss. Rick. - L. 26.7. - Siehe Kap. 5.

P. nanus Pers. - E. (Torp) 7.7. - H. einige $\mathrm{cm}$. breit, runzlig, bräunlich. F. gelblich, gerieft. Sp. $7 \times 6$. Ein schlechtes Ex. Unsichere Bestimmung.

Volvariella plumulosa Lasch - L. 2.8. Sehr selten. H. $4 \mathrm{~cm}$. breit, grau, gerieft-rissig. F. $7 \times 5$, weiss. Schcide aussen russigschwarz. Sp. birnenförmig, $6 \times 4.5$. Dir. W. Ny b e r g zugeschickt.

Amanita vaginata Bull. - Überall auf Aland.

A. vaginata Bull. var. alba Fr. (fungites Batsch, nivalis Grev.)

A. vaginata Bull. var. badia Schff.

A. vaginata Bull. var. fulva Schff. ex. Pers.

A. vaginata Bull. var. lutescens Boud

A. vaginata Bull var. plumbea Schff.

A. muscaria L. - E. L. nebst der Form. aureola L. 3.8.

A. regalis Fr. - Überall. - Früher als A. muscaria.

A. mappa Batsch (citrina Schff.) - E. 18.8.

A. porphyrea A. et S. - E. L.

A. rubescens Pers. - E. H. L.

Macrolepiota procera Scop. - E. H. L.

M. rhacodes Vitt. - L. 12.8 .

M. rhacodes Vitt. var. puellaris Fr. - H. 17.6. H. etwa 5 breit, weiss, feinschuppig. Fl. läuft, nur im $F$. rötlich an. Sp. $8-9 \times 5-5.5$. Úbergänge zu rhacodes. Dir. N y b e r g zugesandt.

Lepiota cristata A. et S. - L. 29.7.

L. clypeolaria Bull. - E.

Agaricus silvaticus Schff. (sanguinarius Karst.) E. H. L.

A. campestris L. - E. H.

A. perrarus Schulz ss. Rick. - Fundstelle nicht notiert. - H. gross, gelbweiss mit strohbräunlichen Schuppen. Scheitel fast glatt, braunfuchsig. F. oben weiss, unten bis zum Ring mit sparrigen bräunlichen Flocken bedeckt, gleichdick, bald hohl. Manschette unten schuppig, zuerst aufsteigend. L. anf. rötlichgrau, dann rotschwarz. Sp. $5.5-6 \times 4.5-5$. Cyst. $15 \times 6$. Fl. läuft überall gelb an. Angen. Geruch. Ich fand einen sehr ähnlichen Pilz auf E. am 9.10.54 und notierte: brauner, schuppiger $\mathrm{H}$. F. hohl. Schaefferreaction positiv, angenehmer Geruch. Sp. c. $6 \times 4$. Schwach gilbend. Wohl nur eine kleinsporige Abart des augustus.

A. arvensis Schff. - E. L. 11.7.

A. silvicolus Vitt. - E.

A. cretaceus Fr. ss. Rick. (A. arvensis var. subexquisitus). - H. im Juli. - Grosser Pilz mit $7 \times 5$ Sp. Ganz problematische Art.
Cystoderma amianthinum Scop. - E.

C. granulosum Batsch - E.

C. cinnabarium A. et S. - E.

Crepidotus fulvotomentosus - H. 29.6. - Versuchsweise bestimmt von Dir. $\mathrm{N}$ y b e r g. C. calolepis ähnlich. Gelbrot, dreieckig, $5 \times 4$. Sp. $8.5 \times 5$.

Rozites caperata Pers. - E. L.

Hebeloma sinapizans Fr. - E. L. 27.7. H.

H. elatum Batsch - E. L. 24.7. - Ähnlich crustuliniforme. Langer cyl. verdrehter F. Sp. $11-$ $13 \times 5.5-7$. Rettichgeruch.

H. crustuliniforme Bull, - E. H. L.

H. longicaudum Pers. - L. 20.7. - Siehe Kap. 4.

H. subsaponaceum Karst. - L. 20.6. - Siehe Kap. 4.

H. sacchariolens Quel. - L. 26.7. Exs.: E. 21.9.54. $\mathrm{H}$. bis 4 breit, bräunlich mit rötlichem Ton, klebrig. Rand heller. F. $7 \times 8$, oliv, bräunlich befasert. L. olivlich. Das Oliv überall mit grünlichem Ton. Sp. $11-12 \times 6.6-7$, citron- bis mandelförmig, grobwarzig, rostbraun. Cyst. auf der L. Schneide keulig, 5.5-7 breit. Unausstehlicher süsser Geruch, etwa wie bei Phol. alnicola.

Inocybe obscura Pers. - H. - Siehe Kap. 4.

I. pusio Karst. - H. L. 25.7. - Siehe Kap. 4.

I. geophylla Sow. - E. L.

I. hystrix Fr. Forma minor Fr. - L. 1.8. - H. 2 breit, dunkelbraun, sparrigschuppig, breit gebuckelt. F. $4 \times 3-4$, sehr dunkel, sparrigschuppig. Sp. $10-10.25 \times 6-6.25$, eiförmig. Cyst. schopfig. Fl. weiss. Geruch gering.

I. delecta Karst. (caesariata Fr. var. fibrillosa). H. L. 8.7. - Siehe Kap. 4.

I. fastigiata Schff. - E. H. L.

I. Cookei Pers. (rimosa Bull.) - H. L. 28.6. - Siehe Kap. 4.

I. descissa Fr. - E. - Siehe Kap. 4.

I. descissa Fr. var. microspora Lange - L. 1.8. $\mathrm{H}$. bis 2 breit, hellcigarrbraun, spitz gebuckelt. Buckel dunkler. F. bräunend. Glatterer $\mathrm{H}$. als Hauptform. Sp. $6.75 \times 4.25$.

I. lacera Fr. - E. H. L.

I. asterospora Quel. - L. 20.7. - Siehe Kap. 4.

I. proximella Karst. - E. L. 9.7. - Siehe Nachtrag c).

I. xanthomelas Kühn. -praetervisa Quel. var. pusilla Lange - H. 1.7. - L. 20.7. - Siehe Kap. 4.

I. subrimosa Karst. - L. 23.7. - Ungeklärter Fall. Ähnlich I. xanthomelas. Sp. ungleich sternförmig $13-14 \times 10-11$.

I. praetervisa Quel. ss. Langei - L. 3.8. - Siehc Kap. 4.

I. napipes Lange - E. H. - Siehe Kap. 4.

I. umboninota Peck (acuta Boud.) - L. 2.8. Siehe Kap. 4.

I. Boltoni Heim (carpta Scop., plumosa (Bolt.) Fr. ss. Quel.) - L. 1.7. - Siehe Kap. 4.

I. lanuginella Schroet. ss. Lange - L. 7.8. - Siehe Kap. 4.

I. putilla Bres. - L. 22.7. - Siehe Kap. 5.

Alnicola scolecina Fr. - L. 4.8. Exs.: E. 14.9.54. - H. bis 2 breit, kast. rostbraun, ausbleichend, nackt. F. rostfarben, bis $6 \times 2$. L. bauchig angewachsen, rostfarben. Sp. $9.5-11 \times 5-6$, zugespitzt. Cyst. mit Schnabel. Zwischen Erlenblättern und Erlenästen. 
Galerina sphagnorum Pers. - L. 29.6. - H. bis 2 breit, zimtwachsgelb, grob gerieft. F. lang, gelblich. Sp. $8-11 \times 6$. Im Moose.

Phaeocollybia Jennyae Karst. ss. Langei - H. 10.6. Siehe Nachtrag c).

Gymnopilus picreus Fr. - E.

G. sapineus Fr. - E. L. 1.7. Exs.: E. 23.9.54. Sapineus, hybridus und penetrans sind schwer auseinanderzuhalten. Einige Charakteristica f. sapineus: Hut filzig bis sammetig-schuppig, rissig zerbrechend. Hyphen der Huthaut $10-18$ im Diam. F. kurz, häufig missgestaltet und grubig gefurcht.

G. liquiritiae Pers. - L. 15.8. - Siehe Kap. 5.

G. penetrans Fr. - E. - Siehe Kap. 4 .

Dermocybe cinnamomea L. - H. L. - Sammelart. Siehe Nachtrag c) und e).

D. malicoria Fr. - L. 30.7. - Siehe Kap. 4.

D. crocea Schff. - E. H. Exs.: 52 und E. 15.9.54.

D. semisanguinea Fr. - E.

Cortinarius violaceus L. - E. 29.8.

C. traganus Fr. - E. L.

C. alboviolaceus Pers.. - E. H. L.

C. camphoratus Fr. - E.

C. suillus Fr. ss. Karst. non Langei - L. 17.8. $\mathrm{H}$. bis 10 breit. bleich lehmfarben, anfangs mit violettem Ton. Rand zuerst seidig. F. $6-7 \times 30$ (unten). Oben zuerst violettlich. Sp. $9 \times 6$, eiförmig oder elliptisch.

C. anomalus Fr. - E.

C. raphanoides Pers. - $\mathrm{H}$.

C. venetus Fr. - E. im Juli. Exs.: E. 12.9.54. Durchweg gelbgrün. H. bis 5 breit, sammetig, glanzlos. F. blasser, faserig. Sp. $6 \times 5$. Geruchlos, mild.

C. callisteus Fr. - L. 13.8. - Siehe Kap. 5.

Hydrocybe armillata Fr. - E. L.

H. evernia Fr. - L. 26.7. - Siehe Nachtrag c).

H. torva Fr. - L. 18.8. - Siehe Kap. 4.

H. erythrina Fr. - L. 3.7. - Siehe Kap. 4.

H. bivela Fr. - L. 13.8. - H. bis 6 breit, fuchsig, kahl, ziemlich glatt, aber nicht unter der Lupe. Nicht eigentlich hygrophan. F. rostblass, schmutzig-weisslich gestiefelt, $10 \times 10$, unten 20 breit. Ringzone undeutlich. Sp. 7-10 5-6 mit körnigem Inhalt.

H. triformis Fr. - L. 16.8. - Siehe Kap. 4.

H. hinnulea Fr. - L. 7.8. - Siehe Kap. 4.

H. brunnea Pers. - L. 30.7.

H. uracea Fr. ss. Karst. - H. Juni. - Siehe Kap. 4.

H. fasciata Fr. (decipiens Pers.) - L. 4.8. - Siehe Kap. 4.

H. Junghuhnii Fr. - H. 14.6. - Siehe Kap. 4.

Myxacium delibutum Fr. - E. L.

M. collinitum Fr. - E. L.

M. mucifluum Fr. - L. 15.8. - Siehe Kap. 5.

M. mucosum Bull. - E. L.

Phlegmacium balteatum Fr. - E. - Siehe Kap. 5.

$P$. arquatum $\mathrm{Fr}$. $-\mathrm{H}$. 2.8. $-\mathrm{H}$. bis 6 breit, wenn älter oliv bis rotbraun. F. $9 \times 10$, violett mit gelbweisslicher Knolle. L. wenn älter zimtfarben, gezähnelt. Sp. mandelförmig $9-10 \times 5.5-6$. Fl. weiss, im F. - besonders oben - violett.

P. multiforme Fr. im Sinne der älteren Deutschen Forscher (Sammelart). Zergliederung von mir erst 1954 versucht. - E. L.

P. triumphans Fr. - E.

$P$. cephalixum Hry, non Secr. (olidum Lange) - L. 16.8. - Siehe Kap. 4.
P. olivascens Batsch ss. Langei - L, 5.8. - Siehe Nachtrag c).

P. percome Fr. - E. Exs.: E. 7.10.54. - H. $5 \mathrm{~cm}$ breit, gelb, zur Mitte zu mehr fuchsig, schmicrig. F. $8 \times 8$ gelb. L. gelb wenn jung, später ziemlich charakterlos, ausgebuchtet. Sp. $12 \times 6.5$, warzig, mandelförmig. Fl. überall ausgesprochen gelb, wird aber rosapurpurn durch $\mathrm{KOH}$. Nadelwald.

P. glaucopus Schff. - E. Exs.: E. 28.9.54. - H. $6-10 \mathrm{~cm}$ breit, braun mit grünlichem Rand. F. kurz mit gerandeter Knolle. L. blau. Sp. $7-8 \times 4.5-5$, elliptisch, eiförmig, auch pflaumenartig, aber nicht mandelförmig. Ohne Geruch und Geschmack.

Conocybe tenera Schff. - E. L.

C. tenera Schff. f. tenella $-\mathrm{H}$.

C. grisea Moser (siliginea Fr. var. typica Kühn.) E. - H. einige $\mathrm{cm}$ breit, graubleich bis isabell. F. verbogen, mit oft verjüngter Basis. Sp. $12 \times 8$, braun. Unsichere Bestimmung.

Bolbitius vitellinus Pers. - L. 1.8 .

Agrocybe praecox Pers. - E.

Pholiota spumosa Fr. - L. 30.7.

P. alnicola Fr. - L. 2.8.

Kuehneromyces mutabilis Schff. - E. H. L.

Naematoloma capnoides Fr. - H.

$\mathcal{N}$. sublateritium $\mathrm{Fr},-\mathrm{H}$.

N. fasciculare Huds. - L.

Stropharia coronilla Bull. - E. L. 7.7. - Siehe Kap. 4.

S. melasperma Bull. - E. 2.7. H. - Siehe Kap. 4.

S. luteonitens Vahl $-\mathrm{L}$. 30.6. $-\mathrm{H}$. bis 3 breit, braun bis honiggelb, konisch-gebuckelt. Abzichbare Haut. F. schlank, dünn, häufig mit langer Wurzel. L. mit Olivton. Sp. $17 \times 10$, oval. Basidien zweisporig.

S. stercoraria Fr. $-\mathrm{H}$.

S. semiglobata Batsch - E. H. L. - Vielleicht identisch mit stercoraria.

Psilocybe coprophila Bull. - E. L. 6.7.

Panaeolus papilionaceus Bull. - E.

$P$. retirugis Fr. - L. 2.7. - Siehe Kap. 4.

P. campanulatus L. - E. L. 14.6.

$P$. acuminatus Schff. - L. 1.7. - Siehe Kap. 4.

P. fimicola Fr. - E. - Siehe Kap. 4.

Panaeolina foenisecii Pers. - E. L. 7.7.

Anellaria separata L. - L. 30.6.

Psathyrella prona Fr. - E. 6.7. L. 6.7. Exs. : 27.9.54.H. 10-15 $\mathrm{mm}$ breit, russfarben mit rötlichem Ton, kahl, 2/3 gerieft. F. $4 \times 1$, blass. L. grau, entfernt. Sp. 13-15 $\times 7-7.5$, elliptisch, undurchsichtig. Waldwege.

P. corrugis Pers. (gracilis Fr. var. corrugis Lange). E. 19.8. - Siehe Kap. 4.

$P$. candolleana Fr. - E. H. L.

Pseudocoprinus disseminatus Pers. - H. 4.6.

Coprinus comatus Fl. Dan. - E.

C. atramentarius Bull.. - E. H.

C. fuscescens Schff. - H. Juni. - Siche Kap. 4.

C. domesticus Pers. (xanthothrix Romagn.) - E. Siehe Kap. 4.

C. micaceus Bull. - $\mathrm{H}$.

C. niveus Pers. - E. - Siehe Kap. 4.

Russula delica Fr. - E. L.

R. pseudodelica Lange - L. 18.7. - Ähnlich delica. L. gelblich. Sp. nur $7 \times 6$ (knapp), feinwarzig, sahnefarben. Riecht. 
R. nigricans Bull. - E. L. 21.7.

$R$. densifolia Secr. - L. 29.7.

$R$. albonigra Krbh. - L. 9. 8. - Zuerst bleich, dann schwarz. Sp. $7.5 \times 6$, kaum warzig.

R. adusta Pers. - E.

$R$. pectinata (Bull.) Fr. ss. Cke - L. 28.7. - H. bis $7 \mathrm{~cm}$ breit, braun. Rand gefurcht. Sp. D. Orn. 2, Grösse 7-10×5.5-7. Geruch unangenehm. Geschmack ziemlich mild.

$R$. foetens Fr, - E. H. L.

$R$. sororia Fr. ss. Schaeffer (consobrina Fr. var. sororia ss. Cooke, pectinata (Bull) Fr. ss. Bres.) - H. Anfang Juli. L. 26.7. - Siehe Kap. 4.

$R$. consobrina $\mathrm{Fr},-\mathrm{H}$.

R. lepida Fr. - E. H. L. - Siehe Kap. 5.

R. lepida Fr. var. lactea (Pers.) Fr. - Siehe Kap. 5.

$R$. heterophylla Fr. f. viridis - L. 22.8.

$R$. vesca Fr. - E. H. L.

$R$. claraflava Grov, - H. L.

$R$. decolorans Fr.. - E. L.

$R$. vinosa Lindbl. (obscura Rom.) - E. H. L.

$R$. xerampelina Schff. var. typica (purpurata Crawsh.) E. H. L.

R. xerampelina Schff. var. Barlae (graveolens Romell) L. 7.8. - Gelboliv, blass.

R. xerampelina Schff. var. graveolens-subrubens Lange H. 5.7. L. 22.7. - Braun.

R. venosa Vel. ss. Melz. - L. 16.7. - Siehe Kap. 4.

R. puellaris Fr. - E. H. L.

$R$. lutea Huds. - E. H. L.

$R$. chamaeleontina Fr. ss. Langei (nauseosa (Pers.) Fr.) - E. H. L.

$R$. aeruginea Lindblad. - E. H. L.

$R$. sp. Lange. - H. Juli. L. 22.8. - Zwischen heterophylla und aeruginea stehend. H. grün, bis 10 breit. Halbabziehbare Haut. L. hellcreme. Sp. $6.5 \times 5.5$, sehr hell. Fl. fest. Sehr problematische Art.

R. paludosa Britz. - E. H. L.

R. aurata With. - L. 4.8. - Dir. Ny berg zugesandt.

R. olivacea Schff. - L. 11.8. - F. rot. Sp. G $-\mathrm{H}$, Orn. 6. Grösse $8-13 \times 8-12$.

R. curtipes Möller et Schaeffer - L. 22.7. - Siehe Nachtrag c).

R. integra L. - H. L. 13.8. - Siehe Kap. 4.

R. Romellii Maire. - E. H. L. - Siehe Kap. 4.

$R$. emetica Schff. - E. L. - Sammelart. Siehe Nachtrag c) und e).

$R$. fragilis Per. (Mairei Singer) - E. H. L. - Kleiner als emetica. $H$. violettlich, entfärbend. Sp. A-B, Orn. 7, Grösse $6-8 \times 5-7$.

$R$. versicolor Schff. $-\mathrm{H}$. - Wie puellaris. L. scharf. Sp. E-F, Orn. 4, Grösse 7-8 ×5.25-5.75.

R. sanguinea Bull. - E. H. L.

$R$. sanguinea Bull. var. rosacea Lange (rosacea (Pers.) Fr. ss. Bres.) - L. 23.7. - Siehe Nachtrag c).

R. sardonia Fr. (drimeia Cke) - H. 13.7. L. 5.8. L. citr.gelb. Äusserst scharf.

R. Queletii Fr. - L. 9.8. - Wie sardonia, aber kleiner und gebrechlicher. F. auffallend rot. L. blass. Sp. G-D, Orn. 5, Grösse 8-7 etc. Sehr scharf. Stachelbeerkompottgeruch.

Lactarius controversus Pers. - E. 28.8. Exs. : E. 11.9.54. - Steht zwischen torminosus und piperatus. Milch weiss, scharf. $\mathrm{H}$. blass mit purpurroten Flecken. F. weiss. L. fleischocker. Sp. $5.5-6 \times 4.5-5$.

L. piperatus Scop.. - $\mathrm{H}$.

L. torminosus Schff. - E. L.

L. scrobiculatus Scop. - E. L.
L. repraesentaneus Britz. - L. 21.7.

L. uvidus Fr. - L. 28.7.

L. uvidus Fr. var. subzonarius Karst. - L. 23.8. Schwach gezont.

L. deliciosus L. - E. H. L.

L. necator Pers. - E.

L. trivialis Fr. - L. 6.7.

L. hysginus Fr. - L. 21.7.

L. lignyotus Fr. - L. 6.8 .

L. glyciosmus Fr. - E. L.

L. flexuosus Fr. - E. H. L.

L. cremor Fr. - L. 28.7. - Siche Nachtrag c).

L. helvus Fr. - E. L.

L. rufus Scop. - E. L.

L. subdulcis Bull. s. lat. - E. H. L. - Sammelart. Siehe Kap. 5.

Cantharellus clavatus Pers. - E. Juli/Aug. - Fk. erst abgestutzt-keulig, violettbraun, zuletzt kreiseloder ohrförmig, bis 8 hoch und 7 breit. Der $\mathrm{H}$. wird bald grünlichgelb. F. kurz und fleischig. L. fleischviolett. Sp. $10-11 \times 4-5.5$, länglichelliptisch, an einem Ende verschmälert. Nadelwald. Dir. Ny berg zugesandt.

C. cibarius Fr. - E. H. L.

C. lutescens Pers. - E. Exs.: E. 11.9.54. - Hymenium und F. dauernd orange. Sp. $9-11 \times 5.5-7$, gelblich. Stark riechend.

Rhizopogon rubescens Tul. - E. H. - F. weiss, in der Luft rötend. Sp. $8 \times 4$

R. rubescens Tul. f. roseolus Corda. - H. - Belanglos.

R. luteolus Fr. - E. - Siehe Kap. 5.

Calvatia caelata Bull. - E. H.

Lycoperdon umbrinum Pers. (hirtum Mart.) - E.

L. gemmatum Batsch - E. H.

L. piriforme Schff. - E. H. L.

Bovista plumbea Pers. - E. L. 26.6.

$B$. nigrescens Pers. - E. H. L.

Phallus impudicus L. - L. 27.7.

Calocera viscosa Pers. (flammea Schff.) - E. L.10.7.

Clavaria botrytes Pers. - E.

C. aurea Schff. - E.

C. flava Schff. - E. L. 21.7.

C. condensata Fr. var. violaceo-tincta. - E. 29.8. Siehe Kap. 4.

C. stricta Pers. - E. - Vielleicht identisch mit C. apiculata Fr.? Strunk dünn und kurz. Fk. fleischgelb mit gelben Endästchen. Sp. $8-9 \times 4$, kurzspindelig, rauhlich, zimtbraun. Bitterlich. Auf Stämmen. Widersprüche in der Lit. Daher Bestimmung unsicher.

C. Invalii Cott. et Wakef., (wohl = abietina Fr.) - E. L. 24.7. - Siche Nachtrag c).

C. lilacina Fr. - E. - Vermutlich var. von C. amethystina (Batt.) Fr.? Fk. lila-purpurn. Sp. $8 \times 5$. Unsichere Art und unsichere Best.

C. pistillaris L. $-\mathrm{E}$.

Stereum hirsutum Willd. - $\mathrm{H}$.

S. rugosum Pers. - H. - Exs.: E. 19.9.54. - Dattelbraun. Auf versch. abgestorbenen Hölzern, besonders Laubhölzern, korkartig ausgebreitet, der stumpfe Rand meist etwas nach oben gebogen. Oberfläche nicht striegelig-filzig. Fruchtlager gelblich, bestäubt, bei Beschädigung rötend. Sp. $8-12 \times 4.5-5.5$. 
Dryodon cirrhatum Pers. - $\mathrm{H}$.

Hydnum repandum L. - E. - L. 16.8 .

H. rufescens Pers. - E. H. L.

Sarcodon imbricatum L. - E. L. 2.8.

S. subsquamosum Batsch non Fr. Quel. - E. 23.8. Siehe Kap. 5.

S. scabrosum Fr. - H. 1.8. - H. bis 8 breit, rostfarbig, filzig, später (nach der Lit.) feinschuppig. F. bis $4 \times 10-15$, unten (auch innen) schwärzlich. St. sehr kurz (im Gegensatz zu S. fennicum $3-5 \mathrm{~mm}$ ), weisslich-grau. Sie laufen am F. etwas herab, sind aber hier nur rudimentär. Sp. 4.5-6×4-5, höckerig, bräunlich. Sehr bitter.

S. sp. - H. 15.7. - H. ziemlich gross, fleischfarben bis violettlich, bisweilen mehrere zusammengewachsen. F. $8 \times 3$, bis an den Grund bedeckt mit rudimentären St. Farbe etwa des H. St. zuerst weisslich, dann bräunlich, am F. z.T. sehr dunkel. Länge der St. sehr ungleich, bis 4 mm. Sp. 3.75-5 im Diam. bräunlich, eckig. Fl. überall weiss und fest, nicht korkig. Geruch angenehm. Geschmack nach Mehl, kaum merklich bitter. Mischwald (überwiegend Fichte). Der Pilz fehlt in der Dir. Ny b e $\mathrm{g}$ und mir zur Verfügung stehenden Literatur.

Calodon suaveolens Scop. - H. L. 23.8.

C. caeruleum Fl. Dan. - E. L. 31.7.

C. aurantiacum A. et S. - E. H.

C. ferrugineum Fr. - E.

C. nigrum Fr. - E. 28.8. - Siehe Kap. 5.

C. cyathiforme Schff. - E. L. 18.8 .

Polyporus ovinus Schff. - E. L. 3.8.

P. confluens Fr. - E. L. 22.8 .

Melanopus squamosus Huds. - Kastelholm 49. Mariehamn 28.6.50. - Lederblass, mit bräunlichen, breiten, fleckenförmigen Schuppen. Sp. $12-$ 15 ×5-6. Auf Laubholzstämmen.

M. melanopus Pers. - E. H. L. - Nadelwaldpilz.

$M$. varius Pers, var. picipes $\mathrm{Fr},-\mathrm{H}$. - Siehe Nachtrag c).
Leucoporus brumalis Pers. - E. H. L.

Leptoporus lacteus Fr. - E. - H. 3-5 breit, weiss, dreieckig. Scharfer Rand. R. weiss. Sp. wurstförmig 3.5-5 × 1.75-2. Auf Laubholzstümpfen.

L. albidus Trog. Schff. (stipticus Quel.) - L. 26.7. Siehe Kap. 5.

L. amorphus Fr. - H. - Siehe Nachtrag c).

L. adustus Willd. - $\mathrm{H}$.

Phaeolus rutilans Pers. (nidulans Fr). - L. 16.7. Siehe Nachtrag c).

Coriolus pubescens Schum. - L. 18.7. -- Siehe Kap. 4.

C. velutinus Pers. ss. Rick. - H. L. 4.7. - Siehe Kap. 4.

C. hirsutus Schrad. - L. 25.7.

C. zonatus Nees - E. H. L.

C. versicolor L. $-\mathrm{H}$. L. 5.7

C. abietinus Dicks. - L. 24.7. - Siehe Kap. 4.

C. connatus Fr. (Fomitopsis c.) - Yppernäs bei Mariehamn. 1.7.50. - H. 3 bis 7 breit, dreieckig, weiss, zottig, später oft von Moosen überzogen, treppenförmig-dachziegelig verwachsen. Auf lebender, alter Eller.

Lenzites betulina L. - E. H.

L. sepiaria Wulf. - E. H. I

Trametes cinnabarina Jacq. - E.

T. odorata Wulf. - H. L. 20.7.

T. serialis Fr. - H. Exs.: Åbo Mai 54. - H. 1-3 breit und $3-6 \mathrm{~m} / \mathrm{m}$ dick. Im ersten J. weiss, dann gelb mit weissem, stumpfem Rand, grubig-runzelig, endlich korkig, zäh. R. weiss, ungleich, sehr eng. Sp. 4-6 $\times 2.5$. Auf Nadelholzstümpfen.

Ungulina fomentaria L. - $\mathrm{H}$.

$U$. marginata Fr. (Fomes ungulatus Schff., Polyporus pinicola Fr.) - E. H. L.

U. annosa Fr. - H. - Braun. Sp. $5 \times 4$. An den Wurzeln, besonders der Nadelhölzer.

$U$. resinosa Fr. (fuliginosa (Scop.) Pat., Ischnoderma benzoinum Vahl, Karst.) - L. 22.8.

$U$. betulina Bull. - E. H. L.

Ganoderma applanatum Pers. - H. L. 22.6.

Phellinus igniarius L. - E. H. L.

Xanthochrous perennis L. - E.

\section{ASCOMYCETES.}

Gyromitra esculenta Pers. - E.

Helvella lacunosa Afz. - L. 5.7. - Bläulichgrau. Sp. $14 \times 7$, elliptisch, farblos, mit einem grossen Öltropfen.

H. atra König - E. 8.7. - Schwarz. F. dicht behaart, schwärzlich. Sp. $14-16 \times 7.5-9$, farblos, mit einem grossen Öltropfen. Dir. $\mathrm{N}$ y b e $\mathrm{r} g$ zugeschickt.

Cudonia confusa Bres. - E.

Macropodia bulbosa Hedw. - H. Mitte Juni. - H. $1-2$ breit. Schwarzbraune Scheibe. Aussen grau.
Welliger, scharfer, verbogener Rand. F. grau, mehlig, tief gefurcht, abwärts verdickt. Sp. $16-20 \times 10$, glatt. Dir. N y b. e r g zugeschickt. Otidea bufonia (Pers.) Boud. - L. 17.7. - Rehbraun. Sp. $12 \times 6$, eintropfig. Bestimmung von Dir. $\mathrm{N}$ y b e r g.

Plicaria badia Pers. - H. L. 29.7.

P. repanda Pers. (varia Hedw., Stevensonia Rehm) H. - Siehe Kap. 4.

Lachnea scutellata L. - L. 9.8. - Siehe Nachtrag c). 


\section{Mustila, im Kirchspiel Elimäki}

$\begin{array}{lrll}1948 & 1.5 .-20.5 . & \text { und } & 8.9 .-15.11 . \\ 1949 & \text { März }-7.5 . & & 24.8 .-17.11 . \\ 1950 & 1.6 .-6.6 . & & 30.9 .-8.11 . \\ 1951 & & & 13.9 .-18.9 . \\ 1952 & & & 23.9 .-27.9 .\end{array}$

1953

13.4 .

Mustila ist sehr pilzreich, besonders das Arboretum, das zu den Sehenswürdigkeiten Finnlands gehört. Das Areal des Arboretums von etwa 120 ha besteht in der Hauptsache aus einen Granitplateau, meist bedeckt mit Moränen und von Nadelhölzern (Pinus silvestris L.) bewachsen. Nur etwa 60 ha der Fläche konnten zum Anpflanzen einheimischer und ausländischer Species (meist Nadelhölzer) verwendet werden. Wertvolle
Laubhölzer konnten nur auf einer begrenzten Fläche am Südhange des Plateaus kultiviert werden, wo der Boden besser ist und das Plateau Schutz gewährt gegen die kalten nördlichen Winde. Das Arboretum wurde von Staatsrat A. F. Tigers te d t gegründet und die ersten Pflanzungen erfolgten im J. 1902. Wenn die Funde in Mustila gegenüber denjenigen der Suchstellen $1952-$ 1954 an Reichhaltigkeit zurücktreten, so ist dies darauf zurückzuführen, dass ich nicht die Möglichkeit hatte das Gebiet im Sommer zu untersuchen, auch fehlten mir damals die Kenntnisse, die ich in den letzten Jahren erwarb. Die Folge war, dass viele Arten in Mustila unbestimmt blieben, die für mich heute kein Problem darstellen würden.

\section{BASIDIOMTCETES.}

Suillus elegans Schum.

$S$. luteus L.

$S$. granulatus L.

$S$. bovinus $\mathrm{L}$.

$S$. variegatus Sow.

S. piperatus Bull.

Leccinum rufum Schff. - Sammelart.

L. scabrum Bull. - Sammelart.

Boletus edulis Bull.

Tylopilus felleus Bull.

Xerocomus badius Fr.

$X$. subtomentosus $\mathrm{L}$.

Paxillus involutus Batsch

$P$. atrotomentosus Batsch

$P$. panuoides Fr.

Hygrophoropsis aurantiaca Wulf.

Gomphidius glutinosus Schff.

G. roseus Fr.

$G$. viscidus L.

Hygrophorus hypothejus Fr.

$H$. erubescens Fr.

H. agathosmus Fr.

H. pustulatus Pers.

H. caprinus Scop.

Camarophyllus virgineus Wulf.

C. niveus Scop. - Herbst 48.

Hygrocybe nitrata Pers.

H. conica Scop.

H. Reai Maire var. insipida Lange - 4.10.50. Siehe Kap. 5.

H. cantharella Schw. -30.8 .49 .

H. miniata $\mathrm{Fr}$.

Lyophyllum putidum Fr. (Tricholoma p.) - 10.10.50. Exs.: E. 3.10 .54 . - H. $2-5$ breit, olivgrau, stellenweise weiss-seidig. F. grau, oft breitgedrückt. L. grau. Sp. $6 \times 3.75$. Stinkt (ranzig).
L. cessans Karst. = Omphalia reclinis Fr.? - Siehe Kap. 5.

L. ulmarium Bull. (Pleurotus u.)

L. connatum Schum. (Clitocybe c.) - Okt. Nov. 50. Sehr blass. H. $5-10$ breit. Viele an der Basis knollig verwachsen. Sp. $5 \times 2.75,5-6 \times 3-4$, $6-7 \times 3.5-4$ etc.

L. decastes Fr. 23.10.49. - Siehe Kap. 5.

Clitocybe nebularis Batsch

C. Alexandri Gill. - 4.10.50. - Siehe Kap. 4.

C. odora Bull.

C. clavipes Pers.

C. inversa Scop. -7.10 .50 .

C. squamulosa Pers.

C. infundibuliformis Schff.

C. cacabus Fr. - Sehr fragliche Art. Identisch mit Cantharellula cyathiformis Bull.?

C. phyllophila Fr.

C. pithyophila Secr. (cerussata Fr. var. pithyophila Lange) - Siehe Kap. 4.

C. cerussata Fr. - Alt beinahe hygrophan. Voller Fuss. Sp. $4-5 \times 3-4$. - Sehr fragliche Art.

C. dealbata Sow. - Herbst 48 .

C. rivulosa Pers.

C. candicans Pers. - Siehe Kap. 4.

C. rhizophora (Vel.) Joss. (Nybergii OvS.) - 30.3.49. und April 1950. - Dir. Nyberg, Mag. Malmström und Prof. Tuomikoski (1954) zugeschickt. Exs.: Lohja 16.5.54. H. bis 2 breit, graubraun, seidig resp. metallisch glänzend, mit gelbem Ton. Eingerollter Rand. Fussbasis mit auffallenden Myzelrhizoiden. F. gleichfarbig, faserig, verbogen, bis 2 lang, voll. L. weisslich bis ockerlich, stark herablaufend. Sp. $4.5-6 \times 2.5-3.25 \mathrm{mit}$ schwachen gelblichen Schimmer. Nadelwald.

C. obsoleta Batsch

C. fragrans Sow.

C. suaveolens Schum. 
C. fritilliformis Lasch. - 1950 wiederholt. - H. bis 4 breit, braungrau, trocken graublass, trichterförmig mit durchscheinend gerieftem Rand. F. meist etwas dunkler als der H., hart. L. grau (wie H.), herablaufend. Sp. $6 \times 3.5$. Der Pilz ist auffallend starr. Chininbitter.

C. vibecina Fr.

C. diatreta Fr.

C. angustissima Lasch - 1950, spät. - Falb. Trocken weiss, kurz gerieft. F. 5-7 lang, dünn, schwankend, elastisch. L. sehr schmal, stark untermischt, sehr gedrängt. Sp. $4.5 \times 3$. Geruchlos. Gesellig.

C. expallens Pers. -1950 wiederholt. - H. 2-3 breit mit bald ausgebreitetem Rand. F. $3 \times 3$. Sp. $4.5-5 \times 3.5-4$ (?).

C. concava Scop. - 7.11.49. - Hygrophan. H. bis 5 breit, braunrussig, trocken grau, tief genabelt, mit welligem ungerieftem Rand. F. kurz, dunkelgrau. L. russbraun, mit Bogen herablaufend. Sp. $9-12 \times 6-8$.

C. dicolor Pers. -5.9 .49 . - Siehe Kap. 4.

C. metachroa Fr.

C. obolus Fr. - Kreisrund. C. vibecina nahestehend. Fragliche Art.

C. brumalis Fr.

Laccaria laccata Scop.

Tricholomopsis rutilans Schff.

Collybia putilla Fr. (Marasmius p.) - Spätherbst. 49 und 50 . - H. bis 3 breit, zimtrot, gerieft. F. zimtpurpurn mit abgebissener, meist weisszottiger Basis. L. ausgebuchtet, mit hellem Rand. Sp. $9 \times 3.75$. Auf Nadeln.

C. confluens Pers.

C. acervata Fr. - 8.9.49. - Siehe Nachtrag c).

C. dryophila Bull.

C. asema Fr.

C. butyracea Bull.

C. maculata A. et S.

Omphatina invita Karst. - 17.10.50. - Hygrophan. H. 3 breit, braungrau, mit eingebogenem Rand, genabelt, glatt. F. 5 lang, Farbe des H. L. schmal und dicht. Sp. $6 \times 3$. Auf verfaultem Holz, weissstriegelig verbunden.

Armillariella mellea Vahl.

Tricholoma flavobrunneum Fr.

T. psammopus Kalchbr. - Jedes Jahr vereinzelt. - H. bis 5 breit, fuchsig-falb. F. mit gelben Schuppen bedeckt. L. bleich, häufig braungefleckt. Sp. $5-6 \times 4-4.5$. Unter Lärchen. Dir. N y b e r $\mathrm{g}$ zugeschickt.

$T$. vaccinum Pers.

T. imbricatum Fr.

$T$. equestre $\mathrm{L}$.

T. inamoenum $\mathrm{Fr}$.

T. album Schff.

T. resplendens Fr. - Sept. 48. - H. bis 8 breit, gelblichweiss, trocken silberglänzend. L. grünlichweiss. Sp. 5-6×3-4. Fl. grünlichblass. Mehlgeschmack, nicht bitter.

T. columbetta Fr. - Herbst 48 und 13.9.51. - H. bis 10 breit, reinweiss, seidig. F. weiss, fas. gestreift. Sp. $6 \times 4$ mit Öltropfen. Mehlgeruch und Mehlgeschmack.

T. saponaceum $\mathrm{Fr}$.

T. portentosum Fr.

T. virgatum $\mathrm{Fr}$.

T. terreum Schff

Cantharellula umbonata Gmel.
C. cyathiformis Bull. (Clitocybe c.)

Melanoleuca strictipes Karst.

M. polioleuca Fr. - 29.8.49.

$M$ arcuata Bull. - Nov. 48 und Sept. 49. - H. bis 8 breit, dunkelolivbraun. F. dick, schwach faserig. L. zuerst weiss, daan ocker, buchtig herablaufend. Sp. $8 \times 5$, rauh.

Catathelasma imperiale Fr. - Ich fand diesen aus Abbildungen in versch. Pilzbüchern wohlbekannten Pilz, der sehr selten ist, in den KivistöFichtenanpflanzungen im Sept. Okt. 48 und an der gleichen Stelle im Herbst 52 und 53. Er ist mit zwei aufsteigenden Ringen versehen. Dir. Nyberg und dem Bot. Institut zugesandt.

Lentinellus bisus Quel. - 3.11.49. - Siehe Nachtrag c).

Pleurotus ostreatus Jacq.

P. salignus Pers. - 23.10.49. - Dieser Pilz blieb anfangs unbestimmt. Siche darüber meine »Seltene Pilze» Seite 13. Vermutlich eine abnorme Form. H. 8 breit, gelbrötlich. F. beinahe $15 \times 20$, schmutzig bräunlich, seitlich. L. weiss, bis 15 $\mathrm{mm}$ breit. Sp. 13-14×5, spulenförmig, sehr regelmässig. Fl. schneeweiss, gummiartig. Nur ein Ex. aus dem Astloch einer lebenden Birke, etwa $1 \frac{1}{2}$ Meter vom Erdboden. Birkenallee.

Panellus mitis Pers.

Panus conchatus Fr.

Lentinus lepideus Fr.

Hohenbuehelia serotina Schrad.

Flammulina velutipes Curt.

Macrocystidia cucumis Per. - Siehe Nachtrag e).

Micromphale perforans Hoffm.

Pseudohiatula esculenta Wulf. - Sammelart. Siehe Kap. 1 .

Marasmius scorodonius Fr.

$M$. oreades Bolt.

$M$. androsaceus L.

M. recubans Quel. - 10.11.49. - H. 2-3 mm breit, schneeweiss, gefurcht. L. nur 6 durchgehende, angewachsen. F. $1-2 \times 0.2$, oben weiss, unten braun, schwach haarig. Sp. spulenförmig, $10-12 \times$ 4.5-5. Auf Blättern.

Xeromphalina campanella Batsch

$X$. cauticinalis With. (Marasmius fulvo-bulbillosus Romell) - Okt. 48. - Dir Nyberg zugeschickt. Wie $X$. campanella, aber auffallend wohlriechend.

Fayodia striaepilea (Fr.) Gill. ss. Karst., Lange (Omphatia s.) -48 . und 31.10 .49 . - H. bis 3 breit, oliv, ausbleichend, mit rippig-gerilltem Umfang. F. abwärts verjüngt. Sp. $5-6 \times 4-5$. Ziemlich geruchlos. Nadelholzwald, in Massen.

F. maura Fr. - H. bis 5 breit, olivschwarz. Trocken silbergrau. F. starr, fast hornartig, kurz, innen und aussen russbraun. L. weisslich, schwach herablaufend. Sp. 5-6×4. Cyst. ziemlich spitz und schmal.

Mycena elegans Pers. (aurantiomarginata Fr.)

M. vitilis Fr. ss. Schroet., Rick. - Siehe Kap. 3.

M. metata Fr.

M. galericulata Scop.

$M$. parabolica Fr. non ss. Lange - 23.10.49. - Viell. identisch mit $M$. maculata Karst. Siehe Nachtrag e). H. 1-2 breit, parabolisch, dunkelgrau mit schwarzem Scheitel, gerieft, nie ausgebreitet. F. beinahe schwarz. L. weissgrau, dicht. Sp. $8-9 \times 5-6$, eiförmig. Büschelig an und bei Stümpfen. 
M. epipterygia Scop.

$M$. vulgaris Pers.

M. citrinomarginata Gill. - Herbst 48.

$M$. rosella Fr.

$M$. pura Pers.

M. polygramma Bull. - Herbst 48.

M. ammoniaca Fr. ss. Langei - 25.10.49. - Viel leicht = aetites Fr. ? Siehe Kap. 5. H. bis 3 breit, hygrophan, glockenförmig, später ausgebreitet, dunkelgrau, beinahe schwarz in der Mitte. 'Trocken viel heller und gefurcht (bis zur Hälfte). F. kurz, glatt, grau $4 \times 2$. Bisweilen viel länger. L. weisslichgrau, entfernt. Sp. $9.5 \times 5.5$. Schwacher alkalischer Geruch. Offene Stellen.

M. alcalina Fr.

M. flavoalba Fr.

M. lactea Pers. var. pitya Fr. -13.10 .49 . - H. $5 \mathrm{~mm}$ breit, weiss, gerieft, mit Höcker. F. bereift, mit striegeligem Knöllchen den Nadeln aufsitzend. L. gedrängt. Sp. $9 \times 3$ mit einem spitzen Ende. Herdenweise.

Baeospora myosura Fr. - Siehe Nachtrag e).

Lepista sordida $\mathrm{Fr}$.

L. nuda Bull.

Clitopilopsis mundula Lasch (Paxillus lepista Fr., Lepista truncata Pers.) - 14.9.51. - H. bis 10 breit, derb, schmutzigweiss. F. fest, bis über 20 dick, mit hornartiger Haut. L. schmutzigweiss, nachdunkelnd, dicht, herablaufend, schmal. Sp. $7-7.5 \times 4-4.5$, ziemlich glatt (nur schwachwarzig), blassgelblich. Geruch von $C$. prunulus. Bitter. Auf einem Beet in der Baumschule (Nadelhölzer).

Clitopilus prunulus Scop.

C. scyphoides Fr, ss. Lange (Omphalias.) - Siehe Kap. 4. Rhodophyllus jubatus Fr. - 20.10.50. - Siehe Kap. 4.

R. turbidus Fr. - 4.10.50. - Hygrophan. H. bis 7 breit, olivgrau mit geradem, bald gerieftem, fast gespaltenem Rand. F. bis $8 \times 7$, grau, gerieft. Sp. $8 \times 7$, unregelmässig kantig. Geruchlos.

R. clypeatus L. -20.10 .50$

$R$. nidorosus Fr. - Herbst 48 .

$R$. rhodopolius Fr.

R. costatus Fr. - 2.9.49. - Siehe Kap. 4.

$R$. staurosporus Lange

$R$. hirtipes Schum., Lange (mammosus ss. Rick.) Siehe Kap. 4.

$R$. sericeus Bull.

R. junceus Fr. - Herbst 48.

\section{Pluteus cervinus Schff.}

Volvariella pusilla Pers. - 6.6.50. - Bestimmt von Mag. N. M a I m s r ö m. H. bis $15 \mathrm{~mm}$ breit, weisslich. F. weiss, seidig, schwach gerieft. Volva meist 4-teilig. Sp. $6 \times 4-4.5$. Unter Rhododendron.

Amanita vaginata Bull.

A. muscaria $\mathrm{L}$.

A. regalis $\mathrm{Fr}$.

A. virosa Fr. - 13.9.51.

A. mappa Batsch

A. porphyrea $\mathrm{A}$. et $\mathrm{S}$.

A. rubescens Pers.

Macrolepiota procera Scop.

$M$. rhacodes Vitt.

$M$. rhacodes Vitt. var. puellaris -4.10 .50 .
Lepiota cristata A. et S.

L. clypeolaria Bull.

Agaricus bisporus Lange (hortensis Cooke var. bispora) $-21.10 .50$

A. silvaticus $\mathrm{Schff}$.

A. arvensis $\mathrm{Schff}$.

A. silvicolus Vitt.

A. macrosporus Moeller et Schff. - Wie silvicolus. Sp. $10-11 \times 7-8$.

Cystoderma carcharias Pers.

C. amianthinum Scop.

C. granulosum Batsch

C. cinnabarinum $\mathrm{A}$. et $\mathrm{S}$.

Crepidotus mollis Schff. -17.10 .50$

C. variabilis Pers.

Ripartites tricholoma A. et S. - 19.10.50. - Siehe Kap. 5.

Tubaria furfuracea Pers. - Sammelart. Zerlegung 1954 versucht.

Rozites caperata Pers.

Hebeloma crustuliniforme Bull.

$H$. longicaudum Pers.

Inocybe obscura Pers. - 6.6.50.

I. pusio Karst. - Herbst 48.

I. geophylla Sow.

I. geophylla Sow. var. lilacina.

I. dulcamara A. et S. - Siehe Nachtrag e).

I. Cookei Bres.

I. abjecta Karst. - 28.8.49. - Siehe Kap. 5.

I. lacera $\mathrm{Fr}$.

I. asterospora Quel.

I. proximella Karst. - 1.9.49.

I. napipes Lange - 28.9.49.

Galerina hypnorum Schrank.

G. badipes Fr. - 23.10. und 5.11.49. - H. 1 breit, rostgelb, gerieft. F. sehr dunkel rostbraun, unten weiss-schuppig. L. rostgelb. Sp. $9-10 \times 5-6$. Cyst. c. 25 - 30 lang. Auf Nadeln.

G. marginata Batsch

Gymnopilus picreus Fr.

G. sapineus Fr.

G. liquiritiae Pers.

G. hybridus Fr. - Siehe Nachtrag e).

Dermocybe cinnamomea L.

D. malicoria Fr. - 4.10.49. und 50 wiederholt.

D. crocea Schff.

D. semisanguinea Fr.

D. miltina Fr. ss. Quel. et Rick. (phoenicea (Bull.) Maire) - 16.10.50. - Siehe Kap. 4.

D. sanguinea Wulf.

Cortinarius traganus Fr.

C. alboviolaceus Pers.

C. camphoratus Fr.

C. pholideus Fr.

C. caninus Fr. - 1.10.50. - Siehe Kap. 4.

C. anomalus Fr. var. pineti Karst.

C. raphanoides Pers. - 6.9.49.

C. gentilis Fr.

Hydrocybe armillata $\mathrm{Fr}$.

H. saniosa Fr. ss. Rick. Vielleicht identisch mit var. paludosa (Fr.) R.Sch.? - H. bis 5 breit, zimtfuchsig, trocken nur wenig die Farbe ändernd, bisweilen gelbseidig bekränzt, mit auffallendem spitzem Buckel. F. rostfarben, häufig mit gelben Schuppenfasern, daher gelblich erscheinend, bis $8 \times 6$, gewöhnlich kleiner. Sp. $8-9 \times$ $4-5$, mandelförmig. 
H. scutulata Fr. - 14.10.50. - Siehe Nachtrag c).

H. torva Fr. - 8.10.50.

H. tortuosa Fr. - 11.10.50. - Siehe Nachtrag c).

H. urbica Fr. ss. Langei -20.9 .49 . $-\mathrm{H}$. bis 5 breit, lehmfarben mit violettlichem Schimmer. F. weiss mit schmalem, blassem Ring. L. rostfalb. Sp. $7.5 \times 4.5$, eiförmig. Fl. hell, oben im F. violettlich.

$H$. bivela $\mathrm{Fr}$.

H. subferruginea Batsch var. imbuta Fr. - 18.9.51. Siehe Kap. 4.

H. biformis Fr. - 14.10.50. - Siche Kap. 4.

$H$. armeniaca Schff. - 14.9.51.

H. damascena Fr. - 25.8.49. - Siehe Kap. 4.

H. candelaris Fr. - 7.10.50. - Siehe Kap. 4.

H. rigens Pers. - Siehe Kap. 4.

H. hinnulea Fr. - 5.10.50. - Siehe Kap. 4.

H. glandicolor Fr. - 2.10.50. - Siehe Kap. 4.

$H$. brunnea $\mathrm{Fr}$.

H. brunneofulva Fr. - Siehe Nachtrag c).

H. hemitricha Fr.

H. incisa Pers. - Siehe Kap. 4.

H. flexipes Pers.

H. rigida Scop. ss. Langei - Siehe Kap. 4.

H. rubricosa Fr. -7.10 .50 .

H. scandens Fr. - 10.10.50. - Siehe Kap. 4.

H. obtusa Fr.

H. fasciata $\mathrm{Fr}$.

H. acuta Fr.

Myxacium delibutum Fr.

M. collinitum Fr.

M. mucifluum Fr. - 7.9.49.

M. mucosum Bull.

M. vibratile Fr. - 14.9.51.

Phlegmacium balteatum Fr. - 9.9.51.

P. multiforme Fr. im Sinne der älteren deutschen Forscher (Sammelart).

P. triumphans Fr. - Herbst 48 und 1.10.50.

P. varium Fr. - Siehe Kap. 4.

P. decolorans Pers. ss. Cke. - Siehe Kap. 4.

$P$. decoloratus Fr. ss. Langei - 22.10.50. - Siche Kap. 4.

P. orichalceum Batsch - 6.10.50. - Siehe Kap. 4.

Leucocortinarius bulbiger A. et S. - 4.10.50.

Conocybe tenera Schff.

Pholiotina filaris Lange (togularis Bull. var. filaris Fr.) - 14.10.49. - Siehe Kap. 3.

P. teneroides Lange - Herbst 48. - Siehe Kap. 5.

Bolbitius Boltonii Pers. - 21.9.49. und 4.10.50. Siehe Kap. 4.

Pholiota heteroclita Fr. - Siehe Nachtrag c).

$P$. squarrosa Müll.

P. flammans $\mathrm{Fr}$.

P. lubrica Pers, - 15.11.49. - Siche Nachtrag c).

P. spumosa $\mathrm{Fr}$.

P. gummosa Lasch - 3.9.49. - Siehe Nachtrag e).

P. astragalina Fr. - 10.10.50. - Siehe Kap. 5.

$P$. alnicola $\mathrm{Fr}$.

$P$. flavida Schff.

Kuehneromyces mutabilis Schff.

Naematoloma capnoides Fr.

N. sublateritium $\mathrm{Fr}$.

N. fasciculare Huds.

Stropharia depilata Pers. (Hornemannii Weinm.).

$S$. aeruginosa Curt.

S. coronilla Bull. - 24.10.49.

S. melasperma Bull. - Herbst 48.
S. stercoraria Fr. - 17.9.51.

S. semiglobata Batsch

Panaeolus campanulatus L.

Anellaria separata L.

Psathyrella gracilis Fr. - $P$. corrugis nahestehend. $\mathrm{H}$. braun, ohne warzenartige Spitze. L. breit mit roter Schneide. Sp. c. $14 \times 6.5$.

$P$. corrugis Pers.

$P$. velutina Pers. $-P$. lacrimabunda nahestend. $H$. 1.5-10 breit, braun, faserigschuppig. F. $10 \times 8$. Sp. $9-11 \times 6-7$, citronenförmig.

P. lacrimabunda Bull. - Herbst 48 und 4.10.50. H. bis 7 breit, fuchsigbraun, faserig-schuppig. L. schokoladenfalb (scheckig) mit weissem Rand (Cystiden). Sp. beinahe schwarz, 9-10×6.

$P$. Candolleana Fr.

P. subpapillata Karst. -3.10 .50 . - H. bis 4 breit, feinkörnig, dunkelrotbraun, hygrophan. Trocken ockergelb. F. $5 \times 3$, seidenglänzend, blass, oben gepudert und gerieft. L. umbrabraun. Sp. $5 \times 3$, dunkelviolett. Büschelig auf Birkenstämmen.

P. hydrophila Bull. - 4.8.49. - $\mathrm{H}$. bis 5 breit, hygrophan, kastanienbraun mit gelbem schimmer. Randgewebe vergänglich. Sp. $5-7 \times 3-3.5$, bleich. Auf und neben Laubholzstämmen.

P. spadicea Schff. - (Psilocybe s.) - 3.10.50. - Siehe Nachtrag c).

P. obtusata Fr. - 15.9.49. - Siehe Nachtrag c).

Coprinus comatus Fl. Dan.

C. fuscescens Schff. - 5.9.49.

C. fimetarius $\mathrm{L}$.

C. micaceus Bull.

C. stercorarius Bull. - Treibhaus, März 49. - H. bis 3 breit, grau, bloss gerieft. F. meist mit schwarzem Sclerotium. Wurzelnd. Sp. $9 \times 5.5$.

C. velox God. - Treibhaus, März-April 49. - H. 1 bis $3 \mathrm{~mm}$ breit, graulich. F. bis 2 lang, ohne Wurzel und ohne Sclerotium. Sp. dunkelumbra, $9 \times 4.5$.

C. cordisporus Gibbs. (Patouiliardi Quel., papillatus Fr. ex Batsch, angulatus Peck). - Treibhaus, März 49. - Siehe Kap. 5.

Russula delica Fr.

$R$. adusta Pers.

$R$. ochroleuca Pers.

$R$. foetens $\mathrm{Fr}$.

$R$. consobrina Fr.

$R$. lepida Fr.

$R$, heterophylla Fr. f. viridis.

$R$, vesca Fr.

$R$. claroflava Grov.

$R$. decolorans $\mathrm{Fr}$.

$R$. vinosa Lindbl.

$R$. xerampelina Schff, var, typica.

$R$. lutea Huds.

R. mollis Quel. - 12.10.50. - Dem Hels. Bot. Institut übergeben. Siehe Nachtrag c).

$R$. aeruginea Lindbl.

$R$. paludosa Britz.

R. Romellii Maire - 21,10.49.

$R$. emetica Schff. - Sammelart.

$R$. fragilis Pers.

$R$. sanguinea Bull.

R. Queletii Fr. - 7.10.50.

Lactarius controversus Pers. - 24.8.49.

L. torminosus Schff. 
L. resimus Fr. - 26.8.49. - Milch weiss, wird sofort gelb. H. bis 15 breit, blass. F. blass, derb. L. teilweise gegabelt, orangeblass. Sp. $8-8.5 \times$ $6.5-7$, nur punktiert.

L. scrobiculatus Scop.

L. repraesentaneus Britz.

L. deliciosus $\mathrm{L}$.

L. necator Pers.

L. vietus Fr.

L. trivialis $\mathrm{Fr}$.

L. lignyotus Fr. - 11.9.49.

L. glyciosmus $\mathrm{Fr}$.

L. flexuosus $\mathrm{Fr}$.

L. pyrogalus Bull. - 19.10.50. - Siehe Nachtrag e).

L. lilacinus Lasch -27.8 .49 .

L. mitissimus Fr. (aurantiacus Fl. Dan.)

L. helvus Fr.

L. rufus Scop.

L. subdulcis Bull. - Sammelart.

Cantharellus cibarius Fr.

C. infundibuliformis Scop. (tubaeformis Fr.) - Herbst 48 und 23.10.50.

Craterellus cornucopioides L. - 26.8.49.

Rhizopogon rubescens Tul.

R. luteolus Fr.

Nidularia confluens Fr. - 16.11.49. - Gelblich. Lappig zerreissende Peridie. Peridiolen ungefurcht, 1-1.5 $\mathrm{mm}$ breit, linsenförmig. Ohne lange Wurzelfasern. Auf einer Brücke.

N. farcta Roth. - Treibhaus im März-April 49. Fk. grau bis braun, sackartig, 5-12 $\mathrm{mm}$ hoch. Peridie filzig. Die freien Peridiolen linsenförmig, zuerst weiss, dann braun, $1-1.5 \mathrm{~mm}$ breit. Lange Wurzelfasern. Auf dem Erdboden.

Crucibulum vulgare Tul.

Calvatia caelata Bull.

Lycoterdon umbrinum Pers.

L. umbrinum Pers. var. asterospermum Dr. et M. Bestimmung von Dir. $\mathrm{N}$ y b e $\mathrm{r} g$

L. gemmatum Batsch

L. piriforme Schff.

Bovista plumbea Pers.

$B$. nigrescens Pers.

Tremella foliacea Pers. - 10.10.50. - Siehe Kap. 5.

T. encephala Willd. (Naematelia e.) -1 im Diam. rund, schmutziggelb oder blass mit fleischrotem Ton. Sp. $8-10 \times 7-8$, mit Spitze.

Exidia glandulosa Bull. - März-April 49. - Oliv, ganz dem Substrat aufliegend, mit kegelförmigen Warzen bedeckt. Sp. $11 \times 5$, wurstförmig. Auf lebendem Walnuss.

E. Friesiana Karst. - Okt. 49. - Grauoliv, glatt. Fk. gehirnartig. Sp. $12-14 \times 4-5$. Auf Nadelholzborke.

E. saccharina Fr. - 12.10.50. - Kandisbraun. Auf Abies sibirica.

Calocera viscosa Pers.

Clavaria aurea Schff.

C. flava Schff.

G. abietina Pers. - Siehe Kap. 4.

C. Invalii Cott. et Wakef.

C. flaccida Fr. - Siehe Kap. 4.
C. Kunzei Fr. ss. Rick. - 26.8.49. und 23.10.50. Fk. weiss, $3-5$ hoch und $2-3$ breit. Abgerundete, sogar keulig-verdickte Endzweige. Sp. farblos, beinahe kugelig, 6-8 im Diam. Bitterlich. Auf dem Erdboden. B o u rdot et G a l$z$ in geben kleincre. Sp. an.

C. ligula Schff.

C. fistulosa Holmsk. - Fk. blass bis rotbraun. Sp. $14-16 \times 6-7$.

Merulius tremellosus Schrad.

Stereum hirsutum Willd.

S. sanguinolentum Schw.

S. rugosum Pers.

S. purpureum Pers.

Pleurodon auriscalpium L. - 13.4.53.

Hydnum repandum $\mathrm{L}$.

H. rufescens Pers.

Sarcodon imbricatum L.

Calodon suaveolens Scop.

C. compactum Pers. - Olivbraun resp. olivgrau, anfangs mit weissem Filz überzogen, der am Rande bleibt. Bei Berührung rötend. Sp. $5 \times 4$ stach., bräunlich.

C. aurantiacum A. et S.

C. ferrugineum $\mathrm{Fr}$.

C. serobiculatum Fr.

C. cyathiforme Schff.

Phylacteria palmata Scop.

P. terrestris Ehrh. (laciniata Pers.) - Herbst 48.

Polyporus ovinus Schff.

Melanopus varius Pers. (picipes Fr.) - Herbst. 48.

$M$. melanopus Pers.

Leucoporus brumalis Pers.

Spongipellis borealis Vahl (var. spathulata Karst.) Siehe Kap. 5.

Leptoporus lacteus Fr. - Herbst 48.

L. fragilis Fr. $-\mathrm{H}$. bis 5 breit, weisslich, durch Berührung braunfleckig. Sp. $3-4.5 \times 2-3$.

L. mollis Pers. ss. Karst. - H. bis 7 breit, blass bis fleischrot, halbkreisförmig. Ohne F. Nach der Befestigungsstelle beiderseits verdiekt, weich, dünn. R. weiss, bei Berührung rotbraun, unregelmässig (eckig). Sp. 3.25-4 $\times 2.25-2.75$ mit einem Tropfen. Fl. zimt, wohlriechend, zäh, aber schwammigweich. Auf toter Kiefer. Vielleicht identisch mit Spongipellis borealis?

L. albidus Trog., Schff.

Coriolus zonatus Nees

C. versicolor $\mathrm{L}$.

Irpex sinuosus Fr. - Febr. 49. - Bestimmung von Dir. N y b e r g. Siehe Kap. 4.

Lenzites betulina $\mathrm{L}$.

L. sepiaria Wulf.

Trametes odorata Wulf.

T. mollis Sommf. (Daedalea m., Polyporus m.) - 18.4. 51. - Graubraun, gezont, ausgebreitet - resupinat. Fast häutigdünn. P. weissgraulich, difform, daher häufig als Daedalea angesehen). - Sp. weiss 9-11 $\times 3.5-4$. Auf toten Acer nigrum. Bestimmt von Prof. $\mathrm{Kujala}$.

Ungulina fomentaria L.

$U$, marginata $\mathrm{Fr}$

$U$. resinosa $\mathrm{Fr}$.

$U$. betulina Bull.

Ganoderma applanatum Pers. 
Phellinus igniarius L.

Xanthochrous perennis L.

Poria calcea Fr. - Weiss. Subiculum beinahe fehlend. P. 1-2 mm lang. Auf Nadelholzstämmen weit ausgebreitet.

\section{ASCOMYCETES.}

Morchella conica Pers.

M. elata Fr. - März-April 49. Treibhaus. - Spitz. Olivfarbene, weite, längliche Zellen. $\mathrm{H}$. von $\mathrm{F}$. scharf abgesetzt, ihn nicht überragend. F. hohl, längsfaltig, weiss, stark kleiig bestäubt, oben oder am Grunde etwas verdickt. Unter Thujopsis dolabrata. In Finnland kaum zuhause.
Gyromitra esculenta Pers.

$G$. infula Schff.

Cudonia circinans Pers. $-2-3 \mathrm{~cm}$ breit, orangegelb, ruzelig, mit eingerolltem $R$ and. F. $2.5-5 \times 5-7$, häufig längsfurchig. Sp. $36-45 \times 2.5-3$.

C. confusa Bres. - 23.9.52.

Spathularia clavata Pers.

Otidea leporina Batsch

Aleuria aurantia Müll.

Plicaria badia Pers.

P. rebanda Pers.

$P$. vesiculosa Bull.

Lachnea scutellata L.

Sclerotinia tuberosa Hedw.

Rhytisma acerinum Fr. -27.9 .52 .

\section{Das Gebiet Vaanila - Lehmijärvi - Laaksopohja, im Kirchspiel Lohja}

Ich verbrachte in diesem Gebiet die Zeit zwischen dem 2.6. und 1.9. und den 9.9.51. Die Pilzsaison war infolge der anhaltenden Trockenheit sehr ungünstig. Die wichtigeren Funde sind durch Exsikkate belegt, doch ist die Qualität der Exsikkate aus diesem Gebiete wenig befriedigend. Einmal waren die Verhältnisse für wissenschaftliche Arbeiten wenig günstig, zweitens, hatte man nicht selten nur ein Ex. zur Bestim- mung und Konservierung zur Verfügung, wobei auch dieses häufig unbefriedigend war. Ein ungünstiges Pilzjahr bedeutet stets auch Pilze schlechter Qualität (Wurmschäden, Deformierung durch Trockenheit etc.).

$$
\text { Abkürzungen: } \begin{aligned}
\mathrm{V} & =\text { Vaanila } \\
\mathrm{La} & =\text { Laakspohja } \\
\mathrm{L} . & =\text { Gebiet am Lehmijärvi }
\end{aligned}
$$

\section{BASIDIOMYCETES.}

Suillus luteus L. - L. 17.8.

S. granulatus L. - L. 29.8.

S. variegatus Sow. - L. 30.7

S. piperatus Bull. - L. 7.8 .

Leccinum rufum Schff. - V. 9.7. - Sammelart.

L. scabrum Bull. - V. 1.7. - Sammelart.

Boletus edulis Bull. - La. 19.7.

Tylopilus felleus Bull. - La. 29.8.

Xerocomus subtomentosus L. - La. 2.8.

Paxillus involutus Batsch - L. 31.7.

P. atrotomentosus Batsch - V. 22.8.

Gomphidius glutinosus Schff. - L. 16.7.

Hygracybe cantharella Schw. - L. 21.8.

Clitocybe geotropa Bull. var. involuta - V. 14.7.

C. infundibuliformis Schff. - La. 29.8.

C. expallens Pers. - La. 14.8.

Laccaria laccata Scop. - L. 23.7.

L. amethystina Bolt. - V. 21.8.

Tricholomopsis platyphylla Pers. (Collybia p.) - V. 7.7.

T. rutilans Schff. (Tricholoma r.) - V. 6.7.

Collybia dryophila Bull. - V. 6.7.

C. aquosa Bull. - V. 9.7. - Siehe Kap. 4.
C. maculata A. et S. - L. 24.8

C. distorta Fr. - L. 16.8. - Siehe Kap. 5.

Armillariella mellea Vahl - V.5.6.

Tricholoma flavobrunneum Fr. - V. 23.8.

T. imbricatum Fr. - L. 16.8 .

T. inamoenum Fr. - V. 23.8.

Melanoleuca strictipes Karst. - V. 22.6.

Panus conchatus Fr. - La. 18.7.

Lentinus lepideus Fr.. - V. 2.7.

Micromphale perforans Hoffm. - V. 17.6

Pseudohiatula esculenta Wulf. - V. 21.6. - Sammelart. Marasmius candidus Bolt. - L. 1.9. - H. bis 1 breit, weiss, durchscheinend, am Rande runzelig. F. 3 lang, oben 1.5 unten $0.6 \mathrm{~mm}$ breit, blass, unten bräunlich. L. bauchig angewachsen. Sp. $9 \times 3$, lancettförmig. Zäh. Auf Moos.

M. oreades Bolt. - V. 9.7.

M. androsaceus L. - V. 6.7.

Mycena vitilis Fr. ss. Schroeter. Rick. (jodiolens Lund.) - V. 21.8. - H. bis $15 \mathrm{~mm}$ breit, tief geriftgefurcht, mit fleischlicher Warze. F. bis 10 lang, fadendünn, wurzelnd, weiss behaart. L. weissgrau, verschmälert angewachsen. Sp. sehr unregelmässig $9-12 \times 5-6$.

M. galericulata Scop. - V. 9.7.

$M$. rosella Fr. - La. 30.8 
M. filopes Bull. (vitilis (Fr.) Quel.) - V. 25. 8. Siehe Nachtrag e)

M. alcalina Fr. - V. 13.7 .

Clitopilus prunulus Scop. - La. 26.8.

Rhodophyllus clypeatus L. - L. 30.7.

Pluteus cervinus Schff. - La. 24.7.

$P$. atromarginatus Konr. (nigrofloccosus (Schulz) Fav., umbrosus (Pers.) Bres.) - L. 23.7. - Wie cervinus mit russfarbener L.schneide.

Amanita vaginata Bull. var. badia Schff. - L. 23.7.

A. vaginata Bull. var. fulva Schff. ex Pers. - V. 20.7.

A. vaginata Bull. var. plumbea Schff. - La 17.7.

A. muscaria L. - V. 7.8.

A. regalis Fr. - V. 20.7 .

A. pantherina DG. - Lohja 9.9.

A. virosa $\mathrm{Fr} .-17.8$.

A. porphyria A. et S. - V. 17.8 .

A. rubescens Pers. - V.22.7.

Agaricus arvensis Schff. - L. 16.8.

Crepidotus scalaris Fr. (applanatus Pers. ss. Karst.) V. 27.8. - H. bis 3 breit, ockergelb, nierenförmig. Ohne F. Sp. $5 \times 5$, leicht warzig. Treppenförmig, auf Nadelholzstämmen und verarbeitetem Holz.

Rozites caperata Pers. - V. 15.8.

Hebeloma longicaudum Pers. - L. 18.8.

Inocybe calamistrata Fr. f. gracilis - V. 3.8. - H. 15 mm breit, kaffeebraun mit starren Schuppen bedeckt, kaum buckelig. F. $2-3 \times 2$, braun, sparrig-schuppig, unten verdickt, mit weisslicher, sogar bläulicher Basis. L. cigarrbraun, mit dicklicher, weisslicher Schneide. Sp. $11-12 \times$ 5.5-6, elliptisch, glatt. Keine schopfigen Cyst. Nicht unangenehmer, starker Geruch. Grabenrand.

I. fastigiata Schff. - V. 3.8.

I. abjecta Karst. - V. 6.8 .

I. lacera Fr. - L. 7.8.

I. lacera Fr. f. gracilis - L. 7. 8. - Bis 2 breit, ausgesprochen gebuckelt. F. $5 \times 2.5$, faserig, unten weissfilzig. L. trübbraun. Sp. $12-15 \times 6$, länglich-eiförmig, bisweilen beinanhe keilartig. Gemischter Wald.

I. napipes Lange - L. 17.8 .

I. umboninota Peck. - La. 1.8.

I. lanuginosa Bull. (ovatocystis Bours. et Kühn.) L. 7.8 .

Galerina hypnorum Schrank. - La. 20.8.

Gymnopilus sapineus Fr. - L. 27.8.

Dermocybe cinnamomea L. - L. 22.8.

D. crocea Schff. - L. 27.7.

Cortinarius violaceus L. - La. 23.8.

C. traganus Fr. - L. 16.8.

C. alboviolaceus Pers. - La. 21.8.

C. camphoratus Fr. - L. 16.8 .

C. violaceo-cinereus Pers. - L. 19.8. - Siehe Kap. 4.

C. spilomeus Fr. - La. 30.8. - Siehe Kap. 4.

C. raphanoides Pers. - L. 30.8 .

C. callisteus Fr. - L. 22.8. - Siehe Kap. 5.

Hydrocybe armillata Fr. - L. 16.8.

H. saniosa Fr. var. paludosa (Fr.) R. Sch. - L. 23.8. - Siehe Nachtrag e).

H. castanea Bull. - La. 29.8. - Siehe Kap. 5.

H. subferruginea Batsch - La. 29.8.
H. armeniaca Schff. - L. 19.8 .

H. diluta Pers. ss. Rick. (saturata Lange) - L. 21.8. Siehe Kap. 4.

H. candelaris Fr. - L. 19.8.

H. brunnea Fr. - L. 23. 8.

H. flexipes Pers. - La. 29.8.

H. paleacea Weinm. - La. 31.8. - Siehe Kap. 4.

H. acuta Fr. - La. 21.8.

Myxacium delibutum Fr. - L. 1.9.

M. mucosum Bull. - L. 18.8 .

Phlegmacium crassum Fr. - V. 28.7. - Siehe Nachtrag c).

P. multiforme Fr. im Sinne der älteren deutschen Forscher. Sammelart. - Lohja 9.9.

P. scaurum Fr. ss. Bres. (montanum Kauffm. ssp. europaeum Moser) - L. 18.8. - Siehe Kap. 4.

$P$. infractum Pers. (anfractum Fr., obscurocyaneum Secr.) - La. 29.8. - Siehe Kap. 4.

P. subsimile Pers. ss. Rick. - La. 29.8. - H. bis 8 breit, rötlichbraun, nur feucht, nicht schmierig. F. blass, nirgends violett, braunfaserig, schwachkeulig. L. sehr breit, werden dunkelzimtoliv. Sp. warzig, $7 \times 5.5$, subglobar. Bitter. Dieser Pilz und $P$. infractum sind kaum auseinanderzuhalten.

Conocybe tenera Schff. - L. 17.8.

Pholiotina filaris Lange (togularis Bull. var. filaris Fr.) - V. 6.7. - H. 1 breit, gelbweiss, mit gerieftem Rand. F. bis $3 \times 1.5$, blass, oben weiss bereift, mit kleiner, weisser Knolle und gerieftem, weissem Ring. L. beinahe frei, bauchig. Sp. $9 \times 6$, rostfarben.

Agrocybe praecox Pers. - V. 30.6.

Pholiota heteroclita Fr. - V. 10.7.

P. myosotis Fr. f. minor (Naucoria m.) - La. 29.8. $\mathrm{H}$. bis 3 breit, oliv. F. dunkelfaserig, oben verdickt, über 10 lang und einige mm breit. L. mit Haken angewachsen, schliesslich schmutzigbraun. Sp. $15-18 \times 9-10$. Moor.

P. confragosa Fr. - V. 20.7. - H. bis 5 breit, zimtfarben bis ziegelrot, mit weisslichen Flocken besetzt. F. faserig, unten mit weissen Haaren besetzt (im Exs. leider verschwunden). L. etwa Farbe des H., gedrängt. Sp. 5.5-6×6.5. Auf einem alten Stamme, wohl Fichte.

Kuehneromyces mutabilis Schff. - V. 14.6.

Naematoloma capnoides Fr. - La. 15.6.

$\mathcal{N}$. fasciculare Huds. - La. 4.6.

Stropharia stercoraria Fr. - L. 16.8.

Psathyrella Candolleana Fr. - L. 16.7.

P. spadiceo-grisea Schff. - La. 21.8. - Siehe Kap. 5. Coprinus atramentarius Bull. - La. 30.8.

C. fuscescens Schff. - V. 27.8 .

Russula delica Fr. - La. 27.7.

R. densifolia Secr. - V. 10.7.

R. adusta Pers. - 24.7.

R. foetens Fr. - La. 19.7.

R. consobrina Fr. - L. 17.8 .

R. rosea Quel. - L. 24.8. - H. bis 6 breit, etwas klebrig, mit abziehbarer Haut, rosa, Mitte gelblich. Rand nur schwach sichtbar gerieft. F. $5 \times 15$, weiss, runzelig-grubig. L. weiss, dicht, am F. zum Teil gegabelt. Sp. B. Orn. 8, Grösse bis $9 \times 7.5$

R. vesca Fr. - V. 21.7. 
R. melliolens Quel. ss. J. Schaeffer - L. 16.7. $-\mathrm{H}$. bis 8 breit, hochrot. F. weiss oder rötlich angelaufen. L. weiss. F. L. und Fl. ockerbraun fleckend. Sp. D. Orn. 11 (beinahe rund und glatt), Grösse $9 \times 9$ etc. Honiggeruch. Mischwald.

R. claroflava Grov. - V. 20.7.

R. decolorans Fr. - L. 4.8.

$R$. vinosa Lindl. - L. 23.7.

R. xerampelina Schff. var. typica - L. 16.8.

R. xerampelina Schff. var. Barlae - V.23.7.

R. venosa Vel. ss. Melz. - L. 16.7.

R. puellaris Fr. - V. 7.7.

R. chamaeleontina Fr. ss. Langei - V. 14.7.

$R$. aeruginea Lindbl. - V. 14.7 .

R. paludosa Britz. - L. 20.7.

R. caerulea Pers. ss. Mich. - V. 15.8. - H. bis 7 breit, dunkelpurpurn mit bis zur Hälfte abziehbarer Haut. Fl, unter der Haut mattpurpurrot. F. keulenförmig, weiss, $8 \times 25$, fest, innen schwammig. L. ocker, gewöhnlich gleichlang, am Grunde verbunden. Sp. G. Orn. 7. Grösse $9 \times 8$. Geschmack mild, schliesslich bitterlich. Gemischter Sumpfwald. Var. umbonata stets gebuckelt.

$R$. olivacea (Schaeff.) Fr. - La. 27.8.

R. integra L. - La. 20.8 .

R. alutacea Fr. - La. 20.8 .

R. Romellii Maire - L. 10.8

R. emetica Schff. - V. 15.8

R. fragilis Pers. - V. 6.8.

$R$. sanguinea Bull. var. rosacea Lange - L. 10.8.

R. fusco-vinacea Lange (depallens Fr. ex. Pers. ss. Cke) - La. 27.7. - H. bis 5 breit, dunkel stumpfviolett. F. weiss. L. weiss, eng. Sp. $7.5 \times$ 6.5, weiss, nicht sehr stark stachelig. Tendenz zu grauen. Geruchlos und geschmacklos. Var. von R. atropurpurea Krombh.?

R. Queletii Fr. - L. 23.8.

Lactarius piperatus Scop. - La. 14.8.

L. torminosus Schff. - Lohja 9.9.

L. scrobiculatus Scop. - L. 23.8.

L. uvidus Fr. - L. 21.8 .

L. deliciosus L. - V. 20.7.

L. necator Pers. - L. 26.8 .

L. trivialis Fr. - V. 28.7.

L. glyciosmus Fr. - L. 17.8 .

L. flexuosus Fr. - La. 24.7.

L. helvus Fr. - L. 24.8.

L. rufus Scop. - L. 30.7.
L. subdulcis Bull. - La. 17.7. - Sammelart. Siehe Kap. 5.

Cantharellus cibarius Fr. - L. 23.7.

Lycoperdon piriforme Schff. - V. 4.6.

Clavaria abietina Pers. - L. 22.8.

Stereum hirsutum Willd. - V. 7.6.

S. purpureum Pers. - V. 4.6.

Dryodon diversidens Fr. - L. 31.7. - Hier im Sinne von Bourdot et Galzin aufgefasst. Im Gegensatz zu D. cirrhatum rötend. Sp. blaugrau durch Jod. Hydnum repandum L. - La. 19.7.

H. rufescens Pers. - V. 14.7.

Calodon cyathiforme Schff. - Lohja 9.9.

Phylacteria terrestris Ehrh. - V. 14.6.

Polyporus ovinus Schff. - L. 19.8.

Leucoporus brumalis Pers. - V. 7.6.

Leptoporus albidus Trog - La. 26.7.

L. amorphus Fr. - V. 7.6.

Coriolus velutinus Pers. ss. Rick. - La. 27.7.

C. zonatus Nees - V. 12.6.

C. versicolor L. - V. 4.6 .

C. abietinus Dicks. - V. 4.6 .

Lenzites betulina L. - V. 3.6.

L. sepiaria Wulf. - V. 2.6.

L. heteromorpha Fr. (Trametes h.) - V. 12.6. - Siehe Nachtrag c).

Trametes cinnabarina Jacq. - L. 16.7.

T. odorata Wulf. - V. 4.6.

T. mollis Sommf. - V. 28.7. - Siehe Kap. 2.

Ungulina fomentaria L. - V. 5.6.

U. marginata Fr. - V. 2.6.

$U$. betulina Bull. - V. 8.6.

Ganoderma applanatum Pers. - V. 3.6.

Phellinus igniarius L. - V. 3.6.

\section{ASCOMYCETES}

Gyromitra esculenta Pers. - V. 2.6.

Vibrissea truncorum Schw. - V. 20.6. - Siehe Kap. 4. Plicaria badia Pers. - L. 23.7.

P. brunneo-atra Desm. - V. 3.8. - Siehe Nachtrag c). Sclcrotinia tuberosa Hedw. - V. 2.6.

\section{Gebiet von Matoniemi - Porraskoski - Nerosjärvi, im Kirchspiel Lammi N. N. W. von Lammi Kirchdorf.}

Ich besuchte dieses Gebiet vom 7.6. bis zum 15.9.52., d.h. bis zum plötzlichen Eintritt starker Nachtfröste. 1952 war ein ausgezeichnetes Pilzjahr. Das Gebiet gehört für den Mykologen sicherlich zu den interessantesten des Landes. Dies gilt insbesondere für den schönen Laub- und gemischten Wald des Bauerngutes Matoniemi.
Auch das S.O. Ufer des Nerosjärvi (Nadelwald) erwies sich als sehr ergiebig.

Abkürzungen:

$$
\begin{aligned}
& \mathrm{M}=\text { Matoniemi } \\
& \text { N. }=\text { S.O.Ufer des Nerosjärvi } \\
& \text { P. = Porraskoski }
\end{aligned}
$$




\section{BASIDIOMYCETES.}

Sclerotinia tuberosa Hedw. - V. 2.6.

Suillus luteus L. -25.6 .

S. granulatus L. -26.6 .

$S$. variegatus Sow. -8.8 .

S. piperatus Bull. -28.7 .

Leccinum rufum Schff. - 15.6. - Sammelart.

L. scabrum Bull. - 16.6. - Sammelart.

Boletus edulis Bull. - 11.6.

Tylopilus felleus Bull. - 4.8.

Xerocomus subtomentosus L. - 24.6.

Paxillus involutus Batsch - 29.6.

$P$. atrotomentosus Batsch -22.7 .

Hygrophopsis aurantiaca Wulf. - 11.8.

Gomphidius glutinosus Schff. - 14.7.

G. viscidus L. -22.7 .

Hygrophorus Karstenii Sacc. et Cub. - 30.8.

H. eburneus Bull. - 16.8 .

H. cossus Fr. - 18.8.

H. olivaceoalbus $\mathrm{Fr}-6.9$.

H. erubescens Fr. - 18.8 .

H. pudorinus Fr. - 7.8. - H. bis 10 breit, fleischrötlich bis cinnober, schwach schmierig. F. bis $10 \times 20$, weiss mit beperlter Spitze. L. herablaufend, weiss, zusammengeschnürt, d.h. durch eine Haarkrone verbunden. Sp. $6.5-8 \times 5-6$. Nadelwald.

H. agathosmus Fr. - 22.8 .

H. pustulatus Pers. - 11.8 .

$H$. tephroleucus Pers. ss. Karst. - 27.8. Ähnlich $H$. olivaceoalbus, aber Sporen. 12-13.5 ×7-8. Fragliche Art.

H. caprinus Scop. -4.9 .

Camarophyllus virgineus Wulf. -10.8 .

C. pratensis Pers. - 24.8 .

Hygrocybe nitrata Pers. - 7.9.

H. ovina Bull. (Camarophyllus o.) -15.8 .

H. tristis Pers. (nigrescens Quel. var.) - 11.8. - Ähnlich $H$. conica. $H$. und $F$. zuerst citrongelb. L. graugrün. Alles schwärzend. Kleinsporig, 8-9 $\times$ 4.5-5. Im Freien.

H. conica Scop. -23.7.

H. chlorophana Fr. - 18.6 .

H. psittacina Schff. - 15.8.

$H$. citrina Rea var. glutinipes Lange - M. 13.8. H. bis $15 \mathrm{~mm}$ breit, durchscheinend gerieft, citr. gelb mit Orangeton, klebrig. F. $3 \times 2$, Farbe des Hutes, klebrig. L. gelb, horizontal, etwas ausgebuchtet, angeheftet mit Zahn. Sp. 5.5-7x 3.5-4, oval/kernförmig. Freie Stelle.

H. coccinea Schff. -20.8 .

H. cantharellus Schw. -11.8 .

H. miniata Fr. - 17.7 .

Lyophyllum rancidum Fr. - M. 10.9. - Nur ein Ex. H. 2 breit, grauschwarz, weiss bereift, nass etwas schmirig. F. $4 \times 3$, grauschwarz, glatt, mit striegeliger Wurzel. L. grau, dicht weisspudrig. Sp. 7-8 $\times 4-4.5$. Mehlgeruch. Mischwald.

L. infumatum Bres. - N. 17.8. - H. 5 breit, olivgrau, erhaben längsrunzelig, mit dunklerem Bukkel, solange jung. F. bis $10 \times 10$, wrzelnd, faserig gestreift. L. mit Zahn angewachsen, langsam an der Schneide schwärzend. Sp. kantig, sehr versch. $8 \times 5.5-6.5,10 \times 6$ etc. Geruchlos. Nadelwald.
L. conglobatum Witt. - 27.7.

L. striaepileum (Fr.) Gill. ss. Karst., Lange - 9.8.

Glitocybe nebularis Batsch - 10.9.

C. Alexandri Gill. (Paxillus extenuatus Fr.) - 23.8. H. 8 breit, auch mehr, tonblass mit glattem Rand. F. bis $8 \times 30$, mit geschwollener Basis. L. schmutzig. Sp. beinahe weiss, $5-6 \times 3.5-4$.

C. odora Bull. - 25.7.

C. odora Bull. var. alba Lange -5.8 .

C. inornata Sow. (Paxillus i.) - P. 20.8. - H. 4 breit, grau bis schmutziglederfarben, fleischig, eingerollt, mit erhaben geripptem Rand. F. Farbe des H., faserig gesreift, knorpelig, $5 \times 6$. L. grau bis schmutzigbraun, aus gerandet-herablaufend. Sp. spulenförmig, farblos, $8-9 \times 3-3.5$. Mehl- oder Rettichgeruch.

C. infundibuliformis Schff. - 17.6.

C. sinopica Fr. -8.6 .

C. phyllophila Fr. -4.9 .

C. pithyophila Secr. (cerussata Fr. var. pithyophila Lange) - 23.8. - F. mit striegeliger Basis, wurzelnd, auf Nadeln. Sp. $6-7 \times 4$.

C. cerussata Fr. -31.8 .

C. dealbata Sow. -23.8 .

C. tuba Fr. - M. 4.9. - Nicht hygrophan. H. bis 5 breit, genabelt, im Gegensatz zu Omph. scyphiformis nicht gerıeft. F. weiss, bis 5 lang. L. weiss, sehr gedrängt, ziemlich weit herablaufend, Sp. $4.5-5 \times 3.25-3.75$, eiförmig bis oval, einige mit Spitze.

C. candicans Pers. - 1.9. - H. bis 3 breit, glänzend weiss, nicht genabelt. F. eachsartig, kurz, sfast röhrig. L. sehr kurz herablaufend (mehr oder weniger angewachsen im Gegensatz zu Clitopilus $=$ Omph. scyphoides Fr.), dicht. Sp. $5 \times 3$. Auf Nadeln.

C. candicans Pers. f. phyllophila - 9.9.-Auf Blättern.

C. obsoleta Barsch. - 27.8. - Hygrophan. Sp. $6.5 \times$ 3.25. Schwacher Anisgeruch.

C. fragrans Sow. -13.8 .

C. suaveolens Schum. -8.8 .

C. ditopa Fr. - P. 18.8. - H. bis 5 breit, braungrau, trocken sattgrau, genabelt-gewölbt, ungerieft. Rand schliesslich eingebogen. F. gleichfarbig. L. ebenso. Sp. $3 \times 2.75$. Mehlgeruch. Bitter. Nadelwald.

C. vibecina Fr. -6.9 .

C. dicolor Pers. - 11.9. - Hygrophan. H. 2-5 breit, grau, trocken weisslich. F. zweifarbig: oben weiss, unten scmutzigbräunlich, mit verdickter Basis, oben nicht mehlig, unten weissfilzig. L. blassgrau. Sp. 6-7×4. Geruchlos.

C. brumalis Fr. -26.8 .

Laccaria laccata Scop. - 14.6.

Tricholomopsis platyphylla Pers. - 16.6.

T. rutilans Schff. -22.6 .

Collybia peronata (Marasmius urens Fr.) - 6.8.

C. confluens Pers. -30.6 .

C. dryophila Bull. - 10.6.

C. dryophila Bull. var. funicularis - M. 1.8. - Dunkel mit schwefelfarbigen L.

C. aquosa Bull. - 18.6. - Sehr hygrophan. Grösse von $C$. dryophila. Ganz fleischfarben. Stark ausblassend. Mit im nassen Zustande dicht gerieftem und scharf abstehendem Rand. F. ziemlich kurz, gelblich, ungerieft. L. gelblich. Sp. kernförmig, $5-7 \times 3-4$. Fleisch weiss-wässerig. 
C. asema Fr. - 17.7. - Graue Form der C. butyracea?.

C. butyracea Bull. - 8.8 .

C. maculata A. et S. -9.8 .

Omphalina umbellifera L. - 10.6.

O. philonotis Lasch ss. Karst. - M. 8.8. - H. bis 3 breit, graurussig. Trocken unter der Lupe flockig. Hygrophan. Aufgerichteter, glatter Rand. F. röhrig, bis $6 \times 2$, grau. L. Farbe des H. Sp. $5.5 \times 3.5$. Sumpf.

O. sp. - M. 5.9. - H. 1 breit, tiefgenabelt, ungerieft, braungelb, kahl. F. $2.5 \times 1$, bräunlich, ganz bedeckt mit gelben Schüppchen und mit dichtgelbfilziger Basis. L. gelb. Sp. 6-6.5 ×3.5-4, oval. Auf Borke.

Armillariella mellea Vahl - 11.6.

Tricholoma flavobrunneum Fr. - 24.7.

T. albobrunneum Pers. (striatum Schff.) - M. 15.9. - Mischwald.

T. vaccinum Pers. -31.8 .

T. imbricatum Fr. -7.9 .

T. inamoenum Fr. -10.8 .

T. album Schff. -12.8 .

T. saponaceum Fr. var. zwischen ardosiacum Bres. und napipes Krombh. - 13.8.

T. saponacum Fr. var. ardosiacum Bres. - 13.9 .

T. portentosum Fr. -21.8 .

T. virgatum Fr. -24.8 .

Cantharellula umbonata Gmel. - 26.8.

C. cyathiformis Bull. -8.9 .

Melanoleuca strictipes Karst. - 17.6.

M. melaleuca Pers. (vulgaris Pat.) - 29.6.

Pleurotus ostreatus Jacq. - 7.7.

P. pulmonarius Fr. var. ostreatus - M. 21.8. H. $5 \times 3$, muschel- bis löffelförmig, gelblich, trokken sahnefarben. F. kurz, seitlich, ganz unten filzig. I. weissgelblich, weit herablaufend. Sp. $7-8 \times 4$, weiss, eiförmig. Auf totem Laubholz.

$P$. acerosus Fr. forma? - P. 24.8. - Ganz unsichere Bestimmung. H. bis 1 breit, rotbraun, gerieft. F. unter 1 lang, sehr dunkel, excentrisch. L. heller. Sp. 5-6 × 3.5-4.5. Auf verfaulter Stange.

Panellus mitis Pers. -5.9 .

Panus conchatus Fr. - 7.7.

Lentinus lepideus Fr. - 3.8.

Micromphale perforans Hoffm. - 24.6.

Pseudohiatula esculenta Wulf. - 8.6. - Sammelart.

Marasmius scorodonius Fr. - 22.7.

$M$. oreades Bolt. - 17.6.

M. androsaceus L. -26.7 .

M. epiphyllus Fr. - M. 29.8. - Siehe Nachtrag e).

Xeromphalina campanella Batsch - 14.6.

Mycena haematopoda Pers. - 31.8.

M. galopoda Pers. var. nigra -9.9 .

M. galericulata Scop. - 19.6.

M. rugosa Fr. - 19.6. - H. bis 2 breit, aschgrau, runzelig. F. blass, glatt, ziemlich kurz, mit kurzer, striegeliger, schräger Wurzel. L. buchtig, hakig angewachsen, weissgrau. Sp. c. $10 \times 6$. Einzeln auf Laubholz. Wohl nur Var. oder Form von M. galericulata.

M. epipterygia Scop. - 14.8 .

M. concolor Lange (Omph. picta Fr. var. concolor Lange) - M. 28.7. - H. 1 breit, kappenförmig, gräunlich, grob gerieft. F. $3 \times 1$, heller als H., an der Basis behaart. L. weisslich, mit grauem Ton, breit, angewachsen, mit Zahn herablaufend. Sp. 6-7 $\times 4$, sehr unregelmässig. Auf Nadeln.
M. rosella Fr. -11.9 .

M. pura Pers. - 14.6.

M. leptocephala Pers. - M. 9.9. - H. bis 3 breit, grob gefurcht (bis 2/3), dunkelgrau. F. dunkel, $10 \times 3$, wurzelnd. L. grau, ausgerandet, entfernt. Sp. sehr versch. $11-13 \times 7-9$. Alkalischer Geruch. Mischwald.

M. alcalina Fr. var. chlorinella Lange - M. 14.8. H. 1 breit, grau, durchscheinend gerieft. F. $4 \times 1$. Sp. $7-8 \times 4-4.25$, eiförmig. Grosse, glatte Cyst. Alkalischer Geruch. Laubwald. Auf Erdboden. Wird jetzt meist mit $M$. leptocephala Pers. identifiziert.

M. ammoniaca Fr. - 26.7 .

M. alcalina Fr. - 25.6.

M. flavoalba Fr. - 25.7 .

M. fibula Bull. (Omphalia f.) - P. 24.8. - H. bis 1 breit, gelborange, gerieft, zuerst genabelt, dann kappenförmig. F. $3 \times 1$, gelb mit Orangeton. L. gelblichweiss. Sp. $4-5.25 \times 2.5-3$. Im Moose des Nadelwaldes.

M. sypsea Fr. - P. 9.6. und 16.6. - H. bis 1.5 breit, weiss. Mitte rahmgelblich. Hochglockig. Rand gerieft. Nackt. F. weiss, steif, bis $4 \times 2$, mit haarigfilziger, abgebissener Basis, gleichdick resp. oben verschmälernd. L. hakig angeheftet. Sp. sehr versch., $7-8 \times 4-5$. Dicht rasig.

M. crispula Quel. (Omphalia c.) - M. 15.9. - H. nur wenige $\mathrm{mm}$ breit, geschweift, kraus, weiss, nackt. F. haarfein, flaumig. L. kaum vorhanden (unter 10). Hymenium eher adrigrunzelig. Auf Blättern.

M. sp. - P. 24.8. - H. unter $8 \mathrm{~mm}$ hoch und unter $7 \mathrm{~mm}$ breit, gelb, mit scharfem, braunen Buckel. F. 3 lang und unter 1 breit, gelblich. L. weissgelb. Sp. $6-7 \times 4-5$, oval. Im Walde.

Baeospora myosura Fr. - 25.8 .

Lepista sordida Fr. -9.9 .

Clitopilus prunulus Scop. -25.7 .

C. scyphoides Fr. ss. Langei (Omphalia s.) - M. 23.8. $-\mathrm{H}$. bis 2.5 breit, weiss und seidig, genabelttrichterförmig. F. dünn, kurz, häufig ezcentrisch. L. herablaufend. Sp. 5.5-6×3-3.25, unregelmässig.

Rhodophyllus jubatus Fr. - M. 29.8. - Nicht hygrophan. H. bis 6 breit, mausgrau, haarig-filzig, bald schuppig-zerklüftet und spaltend. F, 6-9 $\times$ 4-7, etwas heller als H. L. russfarben, später mit purpurnem Ton, tief ausgebuchtet. $\mathrm{Sp}$. $10 \times 6$, unregelmässig.

R. clypeatus L. -7.8 .

$R$. nidorosus Fr. - 22.8. - Stinkt.

R. rhodopolius Fr. - 21.7.

$R$. aprilis (Britz.) Romagn. (majalis Lange) M. 4.9. - Siehe Nachtrag c).

R. costatus Fr. - M. 5.8. - Hygrophan. H. bis 4.5 breit, kahl, braungrau, fast glänzend. F. $3 \times 6-$ 10 , gestreift, schmutzigbraun. L. quergerippt. Sp. $7 \times 6-7$, schwach kantig (5 Kanten). Schwacher Mehlgeruch. Wächst oft rasig.

$R$. staurosporus Lange -25.6 .

$R$. hirtipes (Schum.) Lange (mammosus ss. Rick.) M. 12.6. - H. bis 6 breit, olivbraun, durchscheinend gerieft, mit kleinem verschwindendem Höcker. Trocken isabellfarben. F. bisweilen oben stark bereift, bis 12 lang, fast faserstreifig, unten verdickt und weissfilzig. L. beinahe frei, werden bald braun. Sp. sehr versch., schwach kantig, 
$10-13 \times 7-8$, mit grossen Öltropfen, bisweilen mit schiefer Spitze.

$R$. sericeus Bull. -28.6 .

R. junceus Fr. - 19.6.

$R$. limosus Fr. - M. 5.9. - H. bis 2 breit, russfarben, trocken grauseidig. F. blass, knorpelig, mit etwas verdickter, weisszottiger Basis, $2-2.5 \times$ 4. L. grau, bauchig angewachsen, sehr breit. Sp. meist $7 \times 7$, aber auch $9 \times 7$ etc. Mehlgeruch.

R. griseorubellus Lasch. ss. Karst. - M. 10.9. $\mathrm{H}$. bis 3 breit, braungrau, gerieft. Trocken seidig glänzend. F. $7 \times 3$, hellgrau. L. schwach herablaufend, nicht dicht. Sp. $9 \times 7$ etc., bisweilen mit Stielchen, rundlich. Angenehmer Geruch. Ohne Geschmack. Nadelwald.

R. placidus Fr. - M. 22.8. - Siehe auch Nachtrag c). Vielleicht derselbe Pilz. H. bis 2.5 breit, schwarzbraun, faserig auf grauen Grund. F. $3 \times 2-3$, blau, weissfilzig an der Basis. L. zuerst weiss, dann rosa $\mathrm{Sp}$. ganz unregelmässig, $8 \times 6-$ 6.5 etc. Mischwald, auf faulem Laubholzstamm.

$R$. asprellus Fr. -28.7 .

R. lampropus Fr. - 29.8.

R. aemulans Karst. - M. 12.7. - H. 2 breit, faserigschuppig, ungerieft, dunkelbraun. F. $5 \times 2-3$, etwas blasser. L. segmentförmig, breit, undicht, beinahe braun. Sp. $8-9 \times 5.5-6$, sehr unregelmässig. Im sumpfigen Laubwalde, zwischen Moos. Ein weiteres Ex. vom 22.7. (P.) zeigte ff. Abweichungen: H. 1.25 breit. F. 3 lang. L. bleich. Sp. $6-7 \times 5.5-6$. Unsichere Art.

R. minutus Karst. - M. 24.7. - H. etwa 1 breit blassbraun, trocken isabellfarben. Im nassen $\mathrm{Zu}-$ stande gestreift. F. bräunlich, faseriggestreift, oben hell, $5 \times 1$. L. lancettartig angewachsen. Sp. $8-9 \times 7$ etc.

R. rhodocylix Lasch - M. 8.9. - H. bis 1 breit, bräunlichgrau, flockig, genabelt, grobgerieft. F. 2 lang, fadendünn, kahl, grau. L. bogig herablaufend, undicht. Sp. sehr unregelmässig, $8 \times 8$, $10 \times 8$ etc., meist 5 -eckig. Sumpf.

Pluteus cervinus Schff. -18.6 .

P. pellitus Pers. - P. 27.7. - H. bis 7 breit, weiss, mit rötlichem Ton. F. weiss, abwärts schwach verdickt. Knollige Basis. L. sehr breit, weissrötlich. Sp. $6-7 \times 5$. Fl. weich. Auf Laubholz.

Volvariella pusilla Pers. var. biloba Mass. - P. 24.8. - Nadelwald.

Amanita strangulata Fr. - M. 1.9. - H. bis 6 breit, grau, gerieft, bedeckt mit dunkelgrauen, rundlichen Schüppchen. F. $10 \times 10$ grau, bedeckt mit dunkleren Schuppen. Ringartige Bildung dicht über der Knolle. Sp. rundlich, 9.5-11 $\times 8-9$, auch etwas kleinere, globare kommen vor. Mischwald.

A. vaginata Bull. var. badia Schff. -14.7 .

A. vaginata Bull. var. alba $\mathrm{Fr} .-18.8$.

A. vaginata Bull. var. lutescns Boud. - 16.7.

A. vaginata Bull. var. fulva Schff. -24.7 .

A. vaginata Bull. var. plumbea Schff. -29.6 .

A. muscaria L. - 15.7. und var aurea 22.7.

A. regalis $\mathrm{Fr},-28.7$

A. virosa $\mathrm{Fr} .-4.8$.

A. porphyria A. et S. -4.8 .

A. rubescens Pers. - 2.7 .

Limacella illinita Fr. - P. 27.8. - H. bis 6 breit, weisslich und überall schleimig, mit bräunlichem Buckel. F. $8 \times 5$. Sp. 4 im Diam.
Macrolepiota procera Scop. -4.8 .

M. rhacodes Vitt. -7.8 .

M. puellaris Fr. - 7.8 .

Leucoagaricus excoriatus Schff. - M. 5.9. - H. bis 10 breit, blass, mit Haut, die am Rande teilweise mit dem Ring abreisst, so dass der Rand geschunden erscheint. F. blass, mehlig-filzig mit beweglichem einfachem Ring. L. dicht, ganz frei. Sp. $14 \times 8$, oval. Auf dem Felde.

Lepiota naucina Fr. - M. 8.9. - Feld.

L. alba Bres. - M. 14.8. - Wie L. clypeolaria, aber mit $9-13 \times 5-6$. Sp. Laubwald.

L. clypeolaria Bull. -22.7. nebst weisser Form M. 30.6.

Agaricus bisporus Lange - 10.9 .

A. silvaticus Schff. - 1.9 .

A. campestris L. -28.7 .

$A$. arvensis Vitt. - 5.8.

A. silvicolus Vitt. -5.8 .

A. rubellus Gill. - M. 8.9. - H. bis 5 breit, gelblich, aber bedeckt mit rötlichbraunen Fasern, besonders in đer Mitte. F. $4 \times 7$, mit verdickter Basis, weiss, unten gelb. Ring herabhängend, flüchtig. L. rosa, später schmutzigpurpurn, breit, bauchtig, dicht. Sp. $4-5 \times 3$ mit Öltropfen.

A. rusiophyllus Lasch - M. 28.8. - H. 2.5 breit, gelblichweiss, rosa angehaucht, mit fuchsigem Scheitel. F. $3 \times 3$ (oben). Unten 8 breit, mit fast gerandeter Basis, weiss, dann citrongelb. Hängender Ring. L. lebhaft fleischrosa. Sp. $4-4.75 \times$ 3.25-4. Geruch ganz schwach anisartig. Sehr zart. Laubwald.

Cystoderma carcharias Pers. - 27.8.

C. amianthinum Scop. - 14.9 .

C. granulosum Batsch -26.8 .

C. cinnabarinum A. et S. -9.8 .

Tubaria furfuracea Pers. - 14.6. - Sammelart.

T. phaeophylla Karst. - M. 23.6. - H. bis 2 breit, scmutzigbraun, ungerieft, mit weissen, sehr vergänglichen Schleierresten bedeckt, schwach hygrophan. Trocken blasslederfarben und schwach durchscheinend gerieft. F. $5 \times 3$, bisweilen verbogen, auch zusammengedrückt, Farbe des H., unten sehr dunkel mit vergänglichen Schleierresten. L. herablaufend, dicht, schmal, schmutzigbraun, weiss, bewimpert. Sp. $6-7 \times 4-5$, elliptisch, mit Neigung ins Violettliche. Fl. blass, bitterlich. Büschelig auf gedüngtem Boden in der Nähe des Hofes M. - In der Lit. nahezu unbeachtet. Vermutlich nordische Art.

Rozites caperata Pers. - 21.7.

Hebeloma strophosum Fr. - M. 28.7. - H. 3 breit, einfarbig falb, wie angedrückt-haarig, kaum schmierig. F. $4 \times 5$, bleich, längsfädig (weiss), mit deutlichem Ring. Unten dunkler. L. falb. $\mathrm{Sp}$. beinahe glatt, mitt sehr geringem körnigem Inhalt, 8-9 $\times 4.5-5$. Ubergänge zu $H$. mesophaeum!

H. mesophaeum Fr. - 9.9 .

H. fastibile Fr. - P. 27.6. - H. bis 8 breit, falb, schmierig. F. mit sichtbarem Schleier, bis 10 dick, Farbe des H., häufig verbogen, faserig, gestreift. L. blass, später grau-kanel, kaum tränend, ausgerandet, entfernt. Sp. $8-9.5 \times 5-5.5$, ziemlich farblos. Rettichgeruch. Beinahe büschelig.

H. testaceum Batsch - M. 12.6. - H. bis 6 breit, lederfarben, stumpf. F. mit Velumresten, weisslich, $10 \times 7$, unten verdickt, hohl, Basis bräunlich. 
L. zuerst bleich, dann lehmfarben, schliesslich rostfarben. Sp. glatt od. schwach punktiert, elliptisch, 8.5-11 $\times 5-7$. Birkenwald.

H. crustuliniforme Bull. -6.8 .

H. truncatum Schff. - M. 7.8. - H. bis 5 breit, ziegelrot, schmierig, wellig, robust. F. $5 \times 8$, faserig gestreift, unten etwas verdünnt. L. hellbräunlich, abgerundet, bis $4 \mathrm{~mm}$ breit. Sp. $9 \times 4.5$, ziemlich glatt, ei- bis mandelförmig, beinahe gelblich. Schwacher Rettichgeruch. Bitter.

H. longicaudum Pers. - 4.7. - H. 3-4 breit, sehr blass, schmierig. F. weiss, $7 \times 7$, ziemlich nackt, gleichdick, hohl, mit verdickter Basis und mehliger Spitze. L. tonblass, ausgebuchtet, fein gesägt. Sp. $10 \times 6.5$, leicht punktiert, subcitronförmig. Schwacher, meist nicht rettichartiger Geruch. Bitterlich.

H. subsaponaceum Karst. - 11.9. - H. bis 3 breit, blass, mit rötlichem Anstrich, jung weissfilzig. F. $2-3 \times 5$, cylindrisch, feinfaserig, blass, unten braun. L. schwach gesägt. Sp. $8-9 \times 5-5.5$, eiförmig. Geruch Rettich-Seife, ähnlich $\mathrm{Tr}$. saponaceum. Bitterlich.

Inocybe piriodora Pers. - M. 6.8. - H. bis 5 breit, zuerst glockenförmig, daan ausgebreitet mit Bukkel, weisslich, dann gelbbräunlich, faserig gestreift. F. $8 \times 10$, feinfaserig gestreift, weisslich, von unten rötlich-bräunend, cylindrisch. L. sehr dicht, Farbe des H. Sp. breite, zugespitzte Eiform, gewöhnlich $8.5 \times 5.75$. Aromatischer Geruch. Mischwald. An einem Waldwege. Vermutlich Kollektivart.

I. obscura Pers. - 29.6. - H. 2 breit, cigarrbraun, schuppig, meist ziemlich flach, aber auch spitz gebuckelt. F. blasser, faserig. L. blass. Sp. 9-11 $\times 5-6.5$, ei- bis subcitronförmig, unregelmässig. Cyst. breit schopfig, 10-12, flaschenförmig, bräunlich. Riecht häufig wie I. geophylla.

I. cincinnata Fr. - M. 13.8. - H. 1.5 breit, gebuckelt, dunkelbraun, sparrig-schuppig. F. $3 \times 1.5$, violettlich, besonders oben. L. bleich. Sp. $9 \times 5$, eiförmig. Cyst. schopfig. Waldweg.

I. pusio Karst. - 30.8. - H. 2-3 breit, mit Buckel, cigarrbraun, schuppig, wird strahlichrissig. F. $3.5 \times 3$, bräunlich, unten weisslich, oben kaum sichtbar violett und weisspuderig, gewöhnlich verbogen. L. blass, durch Cyst. feingezähnelt. Sp. braun, 8-10×4-6. Cyst. schopfig, nicht braun.

I. geophylla Sow. - 29.7.

I. geophylla Sow. var. lilacina -6.9 .

I. geophylla Sow. var. lateritia Weinm. - M.29.8. Braunrot.

I. delecta Karst. (caesariata Fr. var. fibrillosa) Pöystilä (P) 16.6. - Übergang zu Flammula s. lato. Junge Ex. mit Schleier. H. 2-5 breit, ohne Buckel, schuppig-faserig, ockerfarben. F. $3 \times 6$, gleichdick, faserig. L. mit Zahn angewachsen. Sp. $7-10 \times 5-6$, gelblich bis ockerkanel, bohnen- bis eiförmig. Keine schopfigen Cyst. Waldwege.

I. fastigiata Schff. - 18.7 .

I. Cookei Bres. (rimosa Bull.) - 17.6. - Ähnlich fastigiata. H. 3 breit. F. weiss mit kleiner abgesetzter Knolle, die bisweilen kaum sichtbar ist. Sp. $7-9 \times 4.5-6$, breite Eiform. Keine schopfigen Cyst. Geophyllageruch.

I. descissa Fr. - M. 1.7. - H. 1-2 breit, konischgebuckelt - ausgebreitet. Schliesslich bis zur
Hälfte rissig. F. $3 \times 3$, weisslich, fast gerieft. Sp. $8.5-10 \times 5-5.5$. Keine schopfigen Cyst. Geruchlos. Nirgends eine Spur violett.

I. flocculosa Berk. ss. Mass. - M. 29.8. - H. bis 4 breit, braun, beinahe schuppig im Centrum und beinahe rissig am Rande. F. $5 \times 5$, unten verdickt, blass. Basis weiss. Sp. $9-.5 \times 5.25$, spitze Eiform.

I. lucifuga Fr. - P. 18.8. - H. 4 breit, braun, rund, dann ausgebreitet mit breitem Buckel, faserschuppig. F. $5 \times 5$, blass, gleichdock, kahl. Spitze weissmehlig. L. oliv, bauchig angewachsen bis frei, gedrängt. Sp. $7.5-9.5 \times 4.5-6$, eiförmig, schief zugespitzt. Cyst. und Paracyst. spitz. Fl. weiss. Geophyllageruch. Nadelwald.

I. posterula Britz. ss. Sacc. - M. 1.7. - H. bis 5 breir, konisch-glockig, dann bucklig-ausgebreitet, ocker, bisweilen mit grünem Ton, faserig gestreift. F. weiss mit Ton des H., $8 \times 10$ (unten. bis 12), keulenartig. L. gelblich. Sp. $7.5-8.5 \times$ 4.5-5.5, eiförmig. Mischwald, auf einem Wege.

I. lacera Fr. -20.6 .

I. sp. - M. 25.6. - H. bis 3 breit, faserig-schuppig, nicht strahlig-rissig, gebuckelt, rehbraun. F. $6 \times 5$, verbogen, faserig gestreift, an der Basis weisswollig. L. schmurzig. Sp. $10.5-12 \times 5-5.5$, elliptisch, mit einer abgeflachten Seite. Geruchlos. Leider keine Notizen über die Cyst.

I. asterospora Quel. - 30.6. - H. bis 6 breir, braun, strahlig-rissig. F. rötlichbraun, faserig gestreift, fast gerieft, flaumig bereift, mit abgesetztem Knöllchen, 6-9 $63-5$. L. sehr dunkel kanel. Sp. sternförmig mit meist $5-6$ Spitzen, $8 \times 7$, $9 \times 8,10 \times 8$, meist kleiner als in der Lit. angegeben.

I. grammata Quel. (hiulca Fr.) - M. 25.7. - H. bis 3 breit, konvex mit spitzem Buckel, lehmbraun mit weissen Fäserchen. F. $6 \times 3$, mit abgesetzrem Knöllchen, bereift. L. weisslich, später blass. Sp. $8 \times 8$ etc. Sehr stumpfe Warzen.

I. xanthomelas Kühn. (praetervisa Quel. var. pusilla Lange) - 1.7. - H. bis 2 breit, gebuckelt, dunkel, faserig gestreift. F. unten gelbbraun mit abgesetzter weisser Knolle, oben ziemlich blass. Sp. sternförmig mit stumpfen Warzen, $9-10 \times$ $7-8$.

I. praetervisa Quel. ss. Langei -27.6 . $-\mathrm{H}$. bis 5 breit, löwengelb. F. $6 \times 5$, feinriefelig, weiss. Sp. beinahe sternförmig mit 5-8 Warzen, $10-12 \times$ $7-9$.

I. napipes Lange -7.7 . $-\mathrm{H}$. bis 5 breit, dunkelbraun mit sehr dunklem Buckel. F. dunkel mit nicht abgesetzter Knolle, $6 \times 6$. Sp. $7-9 \times 6$.

I. umboninota Peck. (acuta Boud.) - 29.6. - H. bis 2 breit, brustförmig mit konischer Papille, dunkelbraun, faserig-aufspaltend. F. ziemlich dunkel, $3 \times 3$ mit nicht abgesetzter hellerer Knolle. L. breit bauchig, beinahe frei. Sp. sehr unregelmässig, 9-10 $\times 7$, aber auch $7.5 \times 7.5,8 \times 7$ et.

I. Boltoni Heim. (carpta Scop., plumosa (Bolt.) Fr. ss. Quel.) - 27.6. - H. bis 4 breit, dunkelbraun, schuppig, breitgebuckelt. Rand später nach oben gebogen. F. cylindrisch, $5 \times 8$, Farbe des $\mathrm{H}$, faserig ohne Knolle. L. buchtig angewachsen. Sp. gewöhnlich trapezförmig, aber auch unregelmässig-länglich, $10-12 \times 6-7$. Warzen nicht stark hervorspringend. Gyst. breit, bis 60 lang. Geophyllageruch. 
I. lanuginella Schroet. ss. Lange - 21.6. - H. bis 3 breit, hellbraun, gebuckelt. F. faserig gestreift, $3 \times 2$, ohne Knolle, von unten bräunend. Sp. länglich, oft abgehauen, $9 \times 6$ etc. Nur schwach hervortretende Erhöhungen. Geophyllageruch.

I. lanuginosa Bull. - 27.7 .

Alnicola bohemica (Vel.) Maire et Kühn. (scorpioides Fr. ss. Langei) - M. 14.8. - H. bis 3 breit, nur feucht gerieft, nackt, braun, schwach gebuckelt. F. $5 \times 2$, verbogen, weissfaserig, mit bereifter Spitze, innen bräunlich. L. hoch angeheftet. Sp. $12 \times 7.25$, citr.förmig, rostfarben. Unter Gestrüpp (Birke, Eller, Weide), Sumpf.

Naucoria cerodes Fr. ss. Langei - M. 6.9. - H. bis 2 breit, wachsgelb, kahl, schwach hygrophan. F. $3 \times 1-2$, gelb, unten rostbraun. L. rostgelb, breit angewachsen. Sp. sehr rauh, $9.5-10 \times$ $6-6.5$, ei- bis citronförmig.

$\mathcal{N}$. segestria $\mathrm{Fr} .-\mathrm{M} .7 .9 .-\mathrm{H}$. bis 3.5 breit, hygrophan, schmutzig honigbraun. Trocken lederfarben, seidig, gerunzelt. F. unten weissfilzig, sonst bleich, mit dünner Haut bedeckt, $5 \times 2$, faserig gestreift. L. sehr eng und schmal, Farbe des H. Sp. 9.5-10×5.5-6, ei- bis citronförmig. Gesellig auf einem Waldwege.

Galerina sphagnorum Pers. - 29.6.

G. paludosa Fr. - 19.6.

G. hypnorum Schrank. - 22.7.

G. marginata Batsch -13.9 .

Gymnopilus sapineus Fr. - 11.8.

G. hybridus Fr. - 12.9 .

G. penetrans Fr. - 6.8. - Schwer von sapineus und hybridus zu trennen. Einige Charakteristika: Hyphen der Haut 4-10 im Diameter. Cyst. haarförmig, ohne Kopf. Fl. weisslich.

Dermocybe cinnamomea L. - 4.8. - Sammelart.

D. malicoria Fr. -16.8 . - H. bis 5 breit, olivgelb. F. lebhaft olivgelb. L. olivfuchsig mit citr. gelber Schneide. Sp. $6 \times 4$, eiförmig. Fl. olivgelb. Geschmack angenehm.

D. crocea Schff. - 13.8 .

D. semisanguinea Fr. -16.7 .

D. miltina Fr. ss. Quel et Rick. (phoenicea (Bull.) Maire) - 18.8. - H. bis 5 breit, dunkelrotbraun, etwas gebuckelt. Trocken schwach ausblassend. F. heller, faserig, mit roten Faserschüppchen. L. Farbe des H. Sp. 6.5-7×4.5, eiförmig. Fl. blassbraun.

D. sanguinea Wulf. - 10.8 .

Cortinarius violaceus L. -16.8 .

C. traganus Fr. -27.7 .

C. alboviolaceus Pers. -24.7 .

C. camphoratus Fr. -4.8 .

C. violaceocinereus Pers. - 6.8. - H. bis 6 breit, violettgrau, schuppig-punktiert oder rissig-schuppig. Fl. gleichfarbig, keulig-knollig, $9 \times 12$ (oben) -25 (unten). L. ausgebuchtet, violettbraun. Sp. $7-7.5 \times 4.5$, eiförmig, zugespitzt. Fl. im allgemeinen bräunlich, nur oben im F. violett. Nasser Mischwald.

C. opimus Fr. ss. Rick. - 1.9. - H. bis 10 breit, robust, lederblass mit honigfarbenem Filz überzogen. F. sehr robust, bauchig und ausspitzend, weisslich mit violettlicher Spitze. L. zuerst bläulich, dann tonfarben. Sp. grob granuliert, rauh, warzig, $7.5-9 \times 6-7$. FI. im F. oben violett. Laubwald.

C. pholideus Fr. -17.7 .
C. spilomeus Fr. - P. 9.8. - H. 3 breit, violettlich oder bräunlich, mit wenigen blutroten Schuppen. F. $8 \times 7$, rotschuppig. L. graulila, später blass kanel. Sp. c. $7 \times 6$.

C. spilomeus Fr. var. depauperatus Lange - M. 15.9. H. bis 4 breit, lehmbraun, heller als Hauptform. Sp. 7-9 $\times 6$. Mischwald. Ganz ohne violette Töne.

C. caninus Fr. - M. 30.8. - H. bis 8 breit, derb, violett-fuchsig, aber schnell ziegelrot. F. $10 \times 15$, blass, hohl, häufig mit blasser Ringzone, unten keulig verdickt, mit weissem strumpfartigem Velum. Sp. $8-8.5 \times 6.5-7$, warzig. Fl. blass. Geruchlos. Mischwald.

C. anomalus Fr. -22.7 .

C. raphanoides Pers. -25.8 .

C. gentilis Fr. - 4.8.

G. callisteus Fr. f. limonius - M. 5.7.-Telamonoide Form von $C$. callisteus. Sp. rauh, 8-8.5-5-5.5. Offenbar nicht identisch mit Hydrocybe limonia $\mathrm{Fr}$. Hydrocybe armillata Fr. - 9.8.

H. saniosa Fr. ss. Langei et Moseri - 20.6. - H. bis 2 breit mit spitzem oder stumpfem Buckel, nicht gerieft, beinahe feuergelb-braun. Trocken glänzend gelbbraun. F. $5 \times 3$ mit gelben Fasern bedeckt (auf dunklerem Grund), verbogen. Spitze heller. L. gelbrot. Sp. $8-8.5 \times 5 \times 5.5$, rauh, ei- bis mandelförmig.

H. torva Fr. - 25.6. - Wenig hygrophan. H. bis 8 breit, konvex, fleischig, violettbraun, graufaserig überreift. F. violettlich, kurz und zwiebelknollig, durch ein dauerhaftes weisses Velum, das mit einem weissen Ring endet, gestiefelt. Später verlängert, bis $10 \times 30$. L. abgerundet angewachsen, entfernt, breit und dicklich, zuerst violett, dann kanel. Sp. $8.5 \times 6-6.5$, mandelförmig. Schwacher Geruch von C. traganus oder nur süsslich.

H. tortuosa Fr. - M. 27.7.

H. erythrina Fr. - 9.6. - Sammelart. H. 3-4 breit, kastanienbraun, trocken glänzend fuchsig, nicht stark hygrophan. Flacher, dunkler Buckel. F. $4-9 \times 3-5$, schwach violettlich, seidig überfasert, etwa gleichdick, verbogen. L. bauchig, stets zimtfarben. Sp. $6.5-8 \times 4.5-6$, eiförmig, beinahe glatt.

H. bulbosa Sow. - N. 17.8. - H. bis 5 breit, dattelbraun, sehr hygrophan, trocken scherbenfuchsig, glockig, dann flach. F. bis $6 \times 8$, mit dicker Knolle, blass, weiss gestiefelt. Ring weisslich, flüchtig. L. lehmfarben, bis dunkelkanel. Sp. $7-8 \times 5-5.5$, eiförmig. Fl. blass, in der Basis des Hutes gelblich.

H. triformis Fr, - 16.8. - H. bis 5 breit, rotbräunlich, glanzlos. F. weisslich, unten verdickt, $8 \times 15$. Deutlicher, aber vergänglicher, weisser Ring. Sp. $8.25-9.5 \times 5-6$, glatt, ei- bis mandelförmig.

H. subferruginea Batsch var. imbuta Fr. - 4.8. H. bis 9 breit, bleich rotfarben, ins Violette neigend. Trocken lederfalb. F. derb, blass mit viol. Schimmer, unten derb zwiebelknollig, faserig. Von unten, innen und aussen bräunend. Schleier weiss, schnell verschwindend. L. ausgebuchtet, schliesslich sehr breit, rostbraun. Sp. $7-9 \times 5-6$, mandelförmig. Unangenehmer $\mathrm{Ge}$ ruch und Geschmack. Büschelig. 
H. biformis Fr. - 26.8, - H. 8 breit, kastanienbraun, glänzend, flach gebuckelt. Trocken braungelblich. Schwach hygrophan. Rand weissfaserig. F. $10 \times 10$, cylindrisch, gestreift. Vergänglicher, weisser Ring. L. gekerbt, queradrig. Sp. $8-9 \times 4.5-5$, beinahe glatt, mandel- bis eiförmig.

$H$. armeniaca Schff. -15.8 .

H. diluta Pers. ss. Rick. (saturata Lange) - 22.6. $H$. bis 8 breit, braunrot. Trocken fuchsig und glänzend. Eingebogener Rand. F. blass, ziemlich gleichdick, bis $9 \times 10$, eventuell etwas verdickend, nach unten zu mit zugespitztem Ende. Blasse Cortina. L. breit, satt zimtfarben. Sp. $6 \times 5$, rundlich. Milder Geschmack.

H. damascena Fr. - M. 13.8. - Stark hygrophan. H. bis 9 breit, kastanienbraun. Trocken gelbrötlich. F. walzenartig, weisslich, faserig, unten wenig dicker, häufig verbogen, $9 \times 10$. Schleier weiss, schnell verschwindend. Bisweilen wurzelnd. Sp. $8-8.5 \times 4.5-5$, dickwandig. Fl. bitterlich, durch Phenolanilin rot. Gesellig oder rasig.

H. candelaris Fr. -23.7 . - H. bis 7 breit, fahlbraun, trocken hellfuchsig, glänzend, konisch mit abrupt niedergebogenem Rand. F. blass, $7-10 \times 9$, undeutlich gerieft, wurzelnd. L. linear, fuchsig, breit. Sp. $7-8 \times 5-5.5$, eiförmig.

H. rigens Pers. -12.8 . $-\mathrm{H}$. bis 4 breit, tonbräunlich, trocken lederweiss, kegelig-gewölbt. F. weiss, seidig glänzend, cylindrisch, wurzelnd, $6-12 \times$ 4-12. Sp. $7-8 \times 4-5$, ei- bis mandelförmig.

H. duracina Fr. - M. 21.8. - H. 5 breit mit anfangs stark niedergebogenem, seidigenem Rand. Längs dem Rand schwach erhöhte Randzone, braunrot (violettlich), ausblassend. Starre Haut. F. $9 \times 10$, weiss, mit dicker, harter Haut, faserig gestreift, schwach wurzelnd. L. angewachsen, wässerig kanelfarben. Sp. 8.5 - 10×4.5-6. Grösse sehr wechselnd. Laubwald.

H. bovina Fr. ss. Langei nec Rick. - M. 23.8. $\mathrm{H}$. bis 8 breit, dattelbraun, trocken fuchsig, mit weissen Fasern am Rande. F. $8 \times 10$ (oben) -25 (unten, Volva), bräunlich, über der weissen Gürtelzone weisslich. L. sehr breit und bauchig, hell kanel, schliesslich rostfarben. Sp. 8.25-9.5 5.5-6, dickwandig, mandelförmig, körnig. Mischwald.

H. hinnulea Fr. -11.8 . $-\mathrm{H}$. bis 9 breit, rostbraun, trocken blassfuchsig-ocker, am Rande durchscheinend gestreift, zuletzt häufig längsrissig. F. 4-11 $\times 6-15$, rostbräunlich, mit weissem, später braunem Gürtel, abwärts beinahe verjüngt. L. sehr breit (bis $15 \mathrm{~mm}$ ) und undicht. Sp. etwa $8 \times 5.5$, eiförmig, warzig. Fl. rostfarben. Stinkt.

H. glandicolor Fr. -4.8 . $-\mathrm{H}$. bis 6 breit, dunkel braun, glockig. Trocken rehbraun. Rand zuers bekleidet mit weissen Fäserchen. F. $10 \times 7$, versehen mit gürtelförmigem, abstehendem Velum $\mathrm{L}$. dunkel rotbraun, breit (bis $9 \mathrm{~mm}$ ), ganzran dig. Sp. $7-9 \times 4-5$, mandelförmig bis oval. Fl dunkel. Var. curta mit beinahe schwarzem Buckel. F. $4 \times 5$.

H. punctata Pers. - M. 8.9. - H. bis 2 breit, nie spitzbuckelig, glanzlos, kastanienzimtbraun. F. bis $6 \times 5$, blass, faserig gestreift, mit schuppiger Basis und weisschuppig bis zur weissen Gürtelzone. L. breit. Sp. $9.5-10.5 \times 5-6$, mandelförmig, punktiert. Nadelwald, rasig. Siehe auch Nachtrag c).
H. brunnea Fr. -4.8 .

H. helvola Fr. - N. 4.8. - H. bis 5 breit, rotbraun, trocken heller, gewölbt, später ausgebreitet und rissig. $F$. bis $12 \times 15$, unten und oben verschmälernd, rostbraun und durch ein weissliches Velum gestiefelt. L. sehr breit $(9 \mathrm{~mm})$, ausgebuchtet angewachsen, zimtfuchsig. Sp. $8-10.5 \times 5-5.5$, mandelförmig mit Spitze.

$H$. hemitricha Fr. -27.8 .

H. incisa Pers. - 10.8. - H. bis 3 breit, kastanienfuchsig, trocken blass, mehr oder weniger gebuckelt, zuerst glatt, nie gerieft, aber schuppigfaserig und rissig-auflösend. F. bleich rostfuchsig dünn mit weissem Velum in Gürtelform. Sp. 7-9 $\times 5-5.5$ glatt, mandelförmig, in der Grösse sehr wechselnd. Kollektivart?

H. flexipes Pers. -24.8.

H. paleacea Weinm. - 15.8. - H. bis 3 breit, hoch gebuckelt, ziemlich hellbraun, glanzlos, durch weisse, zottige Schüppchen am Rande seidig. F. bräunlich, dünn. L. hellzimt. Sp. $7.5-9 \times$ $4.25-6$, ei- bis mandelförmig, auch ellipt. bis kernförmig. Nirgends violettlich. Geruch von Pelargonium.

$H$. rigida Scop. ss. Langei - M. 8.8. - Sehr fest. H. 3 breit, gebuckelt, kastanienbraun, glänzend, meist weiss bekränzt. Trocken erheblich heller. Buckel verschwindend. F. braun, weiss überfasert, weiss gegürtelt, verbogen. L. Farbe des H., etwas ausgebuchtet angewachsen. Sp. $6-8 \times$ 4-5, glatt, ziemlich hell. Meist Pelargoniumgeruch.

H. holophaea Lange (vielleicht $=$ milvina Fr. ss. Rick.) - M. 29.8. - H. bis 6 breit, meist ohne Buckel, kastanienbraun, schwach hygrophan, trocken, olivfuchsig. F. bis $8 \times 10$, Farbe des $\mathrm{H}$. (dunkel), keulenartig. Das untere Ende (Keule) schief und 5 breit, weisslich. L. Farbe des H., breit, undicht, ausgebuchtet angewachsen. Sp. $8-9 \times 4.5-5$, mandel- bis eiförmig, schwach punktiert. Mischwald.

H. subbalaustina Hry (balaustina Lange) - N. 31.8. $\mathrm{H}$. bis 9 breit, rostbraun, durch eingewachsene Fasern gestreift. F. wird aussen und innen rostbraun, bis $9 \times 15$. L. rotbraun, sehr breit. Sp. sehr rauh, ei- bis mandelförmig $8.5-10 \times 5.5-6$.

H. uracea Fr. ss. Karst. -21.6 . - H. 3-6 breit, kastanienbraun, bisweilen ins Grüne spielend. Sehr hygrophan. Trocken lederfarben. Rissig faserig. F. faserig gestreift, braun mit heller Basis, wird beinahe schwarz, bis $12 \times 10$. L. breit, bisweilen gesägt mit heller Schneide. Sp. 7.5$8 \times 4.5-5$, rauh punktiert, mandel- bis eiförmig. Ab Frühling in Gruppen.

H. scandens Fr. -29.7 . - H. $1-3$ breit, rostfuchsig, wenn nass mit schwach gerieftem Rand, trocken weisslich-lederfarben, kegelig-flachgebuckelt. F. blass, weiss überfasert, gewöhnlich bis $7 \times 4$, im Moose jedoch sehr langstielig (bis 15). L. ocker. Sp. $7-8.5 \times 4-5$, ei- bis mandelförmig, ziemlich glatt. Nadelwald. Büschelig. Ähnlich einer kleinen $H$. obtusa.

H. obtusa Fr. - 1.9.

H. decipiens Fr. ss. Langei - M. 6.7. - H. 2 breit, ungerieft, kastanienbraun, dunkler gebuckelt. Trocken blasser. Um den Buckel niedergedrückt. F. $5 \times 3$, feinfaserig, häufig verbogen, violettlich. L. rostfarben. Sp. $8 \times 5.5$, eiförmig oder elliptisch, granuliert. Nasser Laubwald. 
H. fasciata Fr. (decipiens Pers.) - 20.6. - H. bis 2.5 breit, kastanienbraun, dunkler spitzgebuckelt, nur durchscheinend gerieft, seidig glänzend. Trocken falb. F. $7 \times 3$, blass mit gelblichen resp. kanelbraunen Fäserchen überkleidet. Basis weisslich. Sp. $8-8.5 \times 5.25$, ei- bis subcitronförmig, schwach rauh. Steht $H$. acuta sehr nahe.

H. junghuhnii Fr. - 22.6. - Schwach hygrophan. H. bis 3.5 breit, dunkelbraun, sammetig mit dunklerem spitzem Buckel, schwach gerieft. Trocken ledergelb. F. $10 \times 5$. L. gelbocker, dann zimtfarben, stark ausgebuchtet. Sp. $6-8 \times$ $4.5-5.5$, eiförmig, nicht sehr warzig. Cyst. an der Schneide, $16-20 \times 9-10$.

H. acuta Fr. -10.8 .

H. sp. - M. 13.9. - Telamoniaartig. H. 2.5 breit, kastanienbraun, trocken rehbraun, glänzend. Buckelig-glockig, bis zum dunkleren Buckel gefurcht-aufspaltend. F. $7 \times 2$, faserig gestreift, hell mit gelblichen Flecken, unten weissfilzig und verdickt. L. breit, ausgebuchtet, frei. Sp. $11-12.25 \times 6.25-6.75$, mandel- oder eiförmig, rauh. Mischwald.

Myxacium delibutum Fr. - 27.7.

M. collinitum Fr. -24.7 .

M. muscosum Bull. - 9.7 .

$M$. vibratile Fr. -27.7 .

M. sp. - N. 17.8. - Leider nur junge Ex. H. 4 breit, anfangs kugelig, klebrig, grüngelb. F. sehr fest, $8 \times 1-2$ (Knolle), klebrig, gelbfleckig, ganz oben weissfaserig. Schleier weiss. L. sehr dicht, blass, mit ganz schwachem violettrötlichem Schimmer. Fl. überall weiss (glasartig). Sp. $8.25 \times 4.5$, mandelförmig. Apfelgeruch. Nicht bitter. Vielleicht = Phlegacium talum?

Phlegmacium nemorense Fr. ex Lange (variicolor Quel. ex Pers. var. nemorense) - P. 27.7. - H. bis 10 breit, derb, dattelbraun im Scheitel, Rand bläulich. F. fest, weisslich, oben in der Jugend mit viol. Ton, cylindrisch, unten aufgeschwollen, aber ohne gerandete Knolle. Schleier blasslila. L. zuerst blasslila, später zimtfalb. Fl. hart, weisslich, mit bläulichem Ton. Sp. $10-11 \times 5-6$. Nadelwald.

P. talum Fr. - M. 5.8. - H. bis 5 breit, braungelb. F. $5 \times 10$, blass, gelblich anlaufend mit weisser, runder, angesetzter Knolle. L. gedrängt, ausgerandet, stets bleich. Sp. $8-10 \times 4.5-5$, mandelförmig. Fl. blass, mit hornartiger Zone über den L.

P. cyanopus Secr. ss. Konr. et Maubl. - M. 19.8. H. bis 8 breit, braungelb. F. violett, $6 \times 10-15$, unbekantete Knolle. L. breit (bis $9 \mathrm{~mm}$ ), grobgekerbt, zuerst violett, dann kanel/schokoladenbraun. S. $8.5-9.5 \times 5-5.5$, mandelförmig. F1. im $F$. violett, im $H$. weisslich. Laubwald. Nicht identisch mit Langes Ph. cyanopus.

$P$. rapaceum Fr. f. majus Lange - M. 17.8. - Nicht gesägte L.Schneide. Sp. ei- bis citronförmig, $9 \times 5.5$, schwach warzig punktiert.

P. multiforme Fr. im Sinne der älteren deutschen Forscher (Sammelart). - 27.7.

P. claricolor Fr. ss. Rick., non Langei - P. 14.9. H. bis 6 breit, gelbbraun, fest, hart, anfangs durch das weissliche Velum zottig-seidig, bald rissig und dann aufbrechend, zuerst kugelig, dann niedergedrückt. F. hart, fest, bis $12 \times 15$, blass, weisszottig gegürtelt. L. anfangs etwas bläulich, dann schokoladenfalb, gekerbt. Sp.
11-12×6-7, citronförmig, warzig. Fl. mit bläulichem Ton. Starker unangenehmer Geruch.

P. triumphans Fr. -29.7 .

$P$. cephalixum Hry, non Secr. (olidum Lange) M. 12.8. - H. bis 6 breit, falb, klebrig. F. keulenartig, unten etwas schuppig. Sp. $10 \times 5.5 \mathrm{mit}$ körnigem Inhalt, mandelförmig. Geruch stark und unangenchm. Phenolreaction karmin.

P. varium Fr. - M. 5.8 . - H. bis 8 breit, auffallend fuchsig (Rand semmelgelb), nie violett, derb, halbkugelig. F. beinahe weiss, zwiebelig-kegelig. Derbe Knolle. L. zuerst violett, dann zimtgelb, breit. Sp. $12 \times 6$, mandelförmig, nicht warzig, aber stark körnig. Fl. weiss, nie violett.

$P$. decolorans Pers. ss. Cke -8.9 . $-\mathrm{H}$. bis 7 breit, citrongelb, sehr schleimig. F. bis $10 \times 10-20$ (Knolle), oben weiss, unten gelblich. L. zuerst violettlich, dann kanel. Sp. $8 \times 7$, punktiert.

$P$. decoloratum Fr. ss. Langei, Schroet. - M. 20.8. Ohne viol Töne. H. bis 7 breit, tonblass, wenn alt faserig. Bald trocken, dünnfleischig. F. bis $10 \times 10$, weisslich, unten gelblich. L. zimtfalb, breit, gekerbt. Sp. $7.5-8.5 \times 6-7$, punktiert.

P. decoloratum var. betuleti Fr. - M. 14.9. - Filzig, mit Knolle.

P. porphyropus Schw. - P. 24.8. - H. bis 8 breit, lehmfarben bis graugelb, ins Braune spielend, klebrig. F. $10 \times 10$, violett, schwach knollig. L. violett. F. L. und Fl. purpurn anlaufend. Sp. $8.5 \times 5$ etc. mandelförmig. Mischwald.

P. subpurpurascens Batsch - M. 8.8. - H. bis 5 breit, in der Mitte blass dattelbraun, zum Rande zu violett. F. $5 \times 10$, violettlich. L. violettlich. F. und L. bei Verletzung intensiv purpurn anlaufend. Sp. $9 \times 5.5$, ei- bis citronförmig. Laubwald.

P. scaurum Fr. ss. Bres. (montanum Kauffm. ssp. europaeum Moser) - 9.8. - H. bis 4 breit, dattelbis russbraun ungetigert, mit dünnem herabgebogenem Rand. F. $5 \times 5-7$ (unten), faserig gestreift, mit Olivton, oben bläulich-grünlich, mit grünem Schleier. Knolle $15 \mathrm{im}$ Diam. bleich. L. oliv, sehr schmal. Sp. $9.5 \times 5.5$, eiförmig

$P$. scaurum Fr. ss. Bres. Eine zweite Form. - N. 9.8. - H. bis 8 breit, wenn nass getigert. F. bis $12 \times 10$, nirgends bläulich oder grünlich. Knolle bis $25 \mathrm{im}$ Diam. Sonst wie oben. Ungeklärter Fall.

P. orichalceum Batsch - M. 5.9. - H. bis 8 breit, kupferrot mit grünem Rand, sehr klebrig. F. $10 \times 20$, gelbgrau, gekantet knollig. L. schwefelgrün, schliesslich zimtoliv. Sp. $11-12 \times 6-6.5$, mandelförmig, warzig, rauh.

P. fulgens A. et S. ss. Langei - M. 29.8. - H. bis 10 breit, seidig resp. feinfaserig, sämisch bis brandgelb. F. fest, $10 \times 12$, gelb, ausbleichend, mit schiefer, abgesetzter Knolle. L. ausgebuchtet, anfangs gelb, dann gelbbraun. Sp. unregelmässig, ei- bis mandel- oder citronförmig, warzigrauh. $8.5 \times 5$ etc. Fl. überall gelb.

$P$. infractum Pers. (anfractum Fr., obscurocyaneum Secr.) - 27.7. - Dunkle L. Warzige, subglobare Sp. $7 \times 5.5$. Bitter.

Conocybe lactea (Lge) Métr. (lateritia Fr.) - M. 15.8. - H. 1.5-2 hoch, isabellblass, dicht gerieft-gerunzelt, cylindrisch, höher als breit. F. weiss $5 \times 1.5$. L. sehr dicht, rostfarben, schmal. Sp. $11 \times 7$, oval.

C. tenera Schff. -19.6 . 
C. tenera Schff. f. tenella -19.7 .

C. Rickenii J. Schaeffer (siliginea Fr.) - M. 9.9. $\mathrm{H}$. bis 1.5 hock, 2 breit, glockig, ungerieft, blass honigfarben. F. gleichdünn, $8 \times 2.5$, Farbe des H. Sp. $15-17 \times 11-12$. Weide.

Bolbitius Boltoni Pers. - 14.8. - Mit gefurchtem Rand und $14 \times 8$ Sporen. Wohl nur Form von $B$. vitellinus.

B. titubans Bull. - 9.7.

B. vitellinus Pers. - 23.6

Agrocybe praecox Pers. - 19.6.

A. vervacti Fr. - M. 22.8. - H. 3 breit, ungerieft, olivbraun. Trocken ledergelb, Mitte dunkler. F. $2.5 \times 4$, blass, schwach faserig gestreift. L. viol.-olivbraun, sehr dicht. Sp. $8.5-9 \times 5.5-6$, eiförmig, schmutzigbraun. Fl. weiss. Wegrand.

Pholiota flammans Fr. - 7.8.

P. tuberculosa Schff. - M. 5.9. - H. 2 breit, gelbfuchsig, Mitte dunkler. Schuppig. F. gelb, faserschuppig, Basis etwas verdickt, bisweilen excentrisch. L. gelbfuchsig, gesägt. Sp. $7-7.5 \times$ $5-5.5$, ei- resp. bohnenförmig, gelb. Bitter. Auf toter Birke.

P. carbonaria Fr. - 14.9.

P. gummosa Lasch - 26.8.

P. alnicola Fr. - 14.6 .

P. pumila Fr. - M. 29.6. - H. bis 2 breit, hygrophan, honigfarben, trocken lederfarben, nicht gerieft. F. $4 \times 3$, hell, mit unvollkommenem Ring. L. bleich, breit angewachsen. Sp. eiförmig, $8-8.5 \times 5.5-6$. Im Walde, kaum auf Holz. Unklare Art.

Kuchneromyces mutabilis Schff. - 8.6.

Naematoloma capnoides Fr. - 11.6.

N. sublateritium Fr. -13.9 .

N. fasciculare Huds. -8.6 .

Stropharia depilata Pers. - 14.9 .

S. aeruginosa Curt. - 29.8 .

S. coronilla Bull. - 26.6. - H. bis 6 breit, ockergelb, nur feucht. F. weiss, in der Mitte mit strahlig-gerieftem Ring. Sp. $8 \times 5$, braunschwarz resp. schwarzviolett. Auffallend weisses, nur im F. gelblichweisses Fl. Rettichgeruch.

S. melasperma Bull. - 22.6. - Sieht S. coronilla sehr ähnlich. H. viel heller (beinahe weiss). F.Spitze gerieft. Gefurchter, unten glatter Ring. Sp. $8-10 \times 4.5-6$, wenn reif sehr dunkel.

S. stercoraria Fr. -20.7 .

S. stercoraria Fr. var. microspora Sch. - M. 8.9. Sp. $12 \times 8$

S. semiglobata Batsch -22.6 .

Psilocybe uda Pers. f. sphagnicola - M. 11.8. - H. 1-2 breit, buckelig, schwach klebrig, ungerieft, lehmfarben. F. bis $10 \times 2$, ohne Ring, rostbraun, zäh. L. violettlich mit hellerer Schneide, bauchig. Sp. $15-17 \times 8-9.5$, violett. Sumpf.

P. merdaria Fr. - M. 21.8. - Gelb, mit blassem F.

P. coprophila Bull. -27.6 .

Deconica atrorufa Schff. - M. 11.6. - H. bis 1.5 breit, rotbräunlich, wenn trocken lederblass, mit gekerbtem Rand. F. knorpelig, $3 \times 1.5$, Farbe des H. L. bauchig, mit Zahn herablaufend, russbraun. Sp. $6-7 \times 4.5-5$, eiförmig. Waldweg.

D. bullacea Bull. - M. 25.6. - H. bis 2 breit, mit klebriger, leicht zu entfernender Haut und gerieftem Rand. Hygrophan. Dunkelbraun. Trocken lederfarben. Blasenförmig. F. bis $3 \times 2$, fa- serig gerieft, unten dunkelbraun, oben gewöhnlich verbreitet. Spitze bereift. L. dreieckig. Sp. $6-8 \times 4.5-6$, eiförmig bis elliptisch. Beim Hofe M., am Wege, auf Holzstückchen etc.

Panaeolus retirugis Fr. - 23.8. - H. bis 3 breit, lehmbraun mit fleischrotem Ton, schwach netzigrippig, kugelig-glockig, mit anhängendem, zerrissenem, gleichfarbigem Velum. F. fleischrot, bereift, bis $10 \times 5$, gleichdick. L. grauschwarz. Sp. $12-15 \times 9-11$, citronförmig, schwärzlich, undurchsichtig. Auf Exkrementen.

P. campanulatus L. -17.6 .

P. sphinctrinus Fr. - M. 24.7. - Wohl Var. von $P$. campanulatus, kleiner. $H$. beinahe schwarz. Hygrophan. Trocken mit gelblichem Ton. Oberfläche etwas seidig. Mit anhängendem, weissem Velum.

P. acuminatus Schff. - M. 14.8. - H. bis 2 breit, parabolisch oder beinahe konisch (Höhe > Breite), schmutzigbraun. Vom Scheitel aus trocknend, wodurch eine dunklere Zone am Rande entsteht. Trocken falb. F. $3-15 \times 1-2$, oben etwas verdickt, unter Lupe stark bereift, unten braun, oben heller. L. weissflockig. Sp. breit citronförmig $11-15 \times 9-11$, schwarzbraun, undurchsichtig. Staub beinahe schwarz.

$P$. subbalteatus $\mathrm{B}$. et $\mathrm{Br} .-9.9$.

P. fimicola $\mathrm{Fr} .-14.6$. - H. bis 4 breit, hygrophan, fast schwarz, im halbtrockenen Zustand mit dunkler Randzone. Trocken grau. F. bereift an der fast gerieften Spitze, bis 7 lang. L. graurussig, weiss bewimpert, bauchig. Sp. 10-13 $\times 7-8.5$.

$P$. fimicola $\mathrm{Fr}$. var. ater Lange $-\mathrm{M}$. 8.6. $-\mathrm{H}$. $1-1.5$ breit, glockig, beinahe schwarz. Trocken schmutzig lehmfarben. F. $2-3 \times 1$, ziemlich dunkel. Sp. $10-11 \times 7-8$, citronförmig bis elliptisch mit braunem Ton.

Panaeolina foenisecii Pers. - 25.7.

Anellaria separata L. - 8.6.

Psathyrella atomata Fr. - M. 26.6. - H. bis 2.5 breit, kaum hygrophan, kaum gerieft, kleiig, graurussig. F. bis 6 lang, oben weiss, unten gelblich, beinahe durchsichtig, fadendünn, an der Spitze kleiig, sehr gebrechlich. L. bisweilen mit weisser Schneide. Sp. $15 \times 7$, undurchsichtig, ziemlich schwarz. Holzplatz.

$P$. corrugis Pers. (gracilis Fr. var. corrugis Lange) M. 5.9. - H. bis 4 breit, purpurfleischfarben bis lederfarben (sehr wechselnd), runzelig. Trocken fast rosenrot. F. weiss, bis $10 \times 2$. L. schmutzigviolett, ohne rote Schneide. Sp. $11-13 \times 7$.

P. Candolleana Fr. - 10.6.

P. subnuda Karst. - M. 2.9. - H. bis 3 breit. Sehr hygrophan. Nass schmutzig braungrau, trocken hell lehmfarben, mit rötlichem Ton, gekerbtgerieft. F. $7 \times 2-4$, weiss, oben gepudert, Basis weissfilzig. L. breit, angewachsen, schmutzig violett. Sp. $8.5 \times 5.5$, undurchsichtig, dunkelbraun mit Purpurton. Laubwald.

Coprinus atramentarius Bull. - 10.9.

C. fuscescens Schff. - 7.7. - Vielleicht nur eine kleine Form von C. atramentarius.

C. fimetarius L. - M. 24.7 .

C. macrocephalus Berk. (fimetarius var. macrocephalus) M. 24.8. - H. 2 hoch, grau, asusbreitend, dabei gelbbraune Spitze und Furchen offenbarend. F. $10 \times 4$, weiss, ohne Wurzel, glatt. Sp. $15 \times 8$, schwarz, undurchsichtig. 
C. lagopus Fr. - M. 25.6. - H. 1-2 hoch, aufschirmend. Dabei verschwindet weissflockige Bedeckung und enthüllt grauen, ausgesprochen gerieften H. F. weiss, zeitweilig flockig, bis 12 lang, nicht wurzelnd. Sp. $10-11 \times 6-7$, schwarz, undurchsichtig. Wächst nicht auf Dünger. Am Hof.

C. domesticus Per. (xanthothrix Romagn.) - 24.7. H. bis 4 breit, russig mit kastanienbraunem Scheitel, anfangs kleiig-schuppig und eiförmig, dann ausgebreitet, wellig gefurcht. F. weiss, seidig glänzend, bis $6 \times 3$. Sp. durchsichtig. braun, $7-9 \times 4.5-6$. Kaum zerfliessend, aber sehr gebrechlich.

C. micaceus Bull. - M. 4.8.

C. niveus Pers. - M. 8.9. - Reinweiss. Sp. 15-12, sehr dunkel, aber nicht schwarz.

C. plicatilis Curt. - M. 10.6. - H. bis 1.5 hoch, braungrau, gefurcht bis zum gelben Scheibchen. Sehr zart. F. blass, verbogen. L. grauschwarz, an einem fleischigen Scheibchen angewachsen. Sp. rund, triangulär oder herzförmig, $10-12 \times 9-10$. Freie Stelle.

Russula delica Fr. - 12.7.

$R$. densifolia Secr. -14.7

$R$. adusta Pers. -22.7 .

$R$. foetens Fe. -27.7 .

$R$. sororia Fr. ss. Schaeffer (consobrina Fr. var. sororia ss. Cke, pectinata Bull. ss. Bres.) - 15.7. - Sieht $R$. pectinata (Bull.) Fr. ss. Cke sehr ähnlich. Abweichungen: Dunkler, scharf. Sp. heller (C.) und kleiner $(7-8 \times 5-6)$

$R$. consobrina Fr. -9.8 .

R. heterophylla Fr. f. viridis -5.7 .

$R$. vesca Fr. - 16.7.

R. cyanoxantha Schff. - M. 7.8. - H. 5-15 breit, schmutziglila, klebrig mit ziemlich leicht abziehbarer Haut. F. weiss $6 \times 10$, cylindrisch. L. ganz weiss, dicht, angewachsen. Sp. A. Orn. 10 Grösse $8 \times 7$. Ohne Geruch. Mild. Laubwald.

R. grisea (Secr.) Gill. - M. 18.7. - H. bis 9 breit, in der Feuchtigkeit schmierig, graugrünlich mit rötlichem Ton. Haut halbabziehbar. Rand später gerieft. F. bis $6 \times 20$, weiss, gleichdick. L. sahnefarben, eng, angewachsen. Sp. G-D. Orn. 7. Grösse 7-8×5-6. Laubwald.

R. claroflava Grov. - 17.7.

$R$. decolorans Fr. -20.8 .

$R$. vinosa Lindbl. -25.8 .

$R$. xerampelina Schff. var. ty pica Crawsh. -5.7 .

$R$. xerampelina Schff. var. Jusca M. et Z. -27.8 . Braun.

$R$, venosa Vel. ss. Melz. (nitida Pers. ss. Kühn. et Romagn.) - 18.7. - H. bis 6 breit, rotgelb mit purpur Ton. Haut zur Hälfte abziehbar. Rand gerieft. F. weiss, gewöhnlich rötlich angehaucht, oben dünner, adrig, von unten her bräunend. L. crème bis schlüsselblumengelb, breit, abgerundet, frei. Sp. E-G, Orn. 6, Grösse 9-11 $\times 7-8$.

R. nitida Pers. ss. Pears. - N. 27.8. $-\mathrm{H}$. bis 6 breit. Rand violett, gerieft. Mitte oliv. Sehr klebrig. Abziehbare Haut. F. weiss. L. crème, dann auffallend eigelb. Sp. G-H, Orn. 6, Grösse 9-10※ 8-8.5. Nur L. scharf. Mischwald.

R. puellaris $\mathrm{Fr}$. -7.7 .

R. lutea Huds. - 15.7 .

$R$. chamaeleontina Fr. ss. Langei -12.7 .

$R$. aeruginea Lindbl, -30.6 .
R. paludosa Britz. - 19.7.

R. aurata With. -15.7 .

R. integra L. -8.7 . - H. bis 12 breit, rotbraun, rot, schokoladenfarben. Haut zur Hälfte abziehbar, klebrig. F. weiss, $3-9 \times 12-30$. L. ocker. Sp. $\mathrm{G}-\mathrm{H}$. Orn. 6, Grösse 9-11 $\times 8-9$, meistens über 10 lang.

R. Romellii Maire - 21.7. - H. bis 15 breit. Sehr dunkel (rot), aber ausbleichend bis gelb. Haut zur Hälfte abziehbar, klebrig, glänzend. F. stets weiss, fest. L. an der Basis häufig verbunden oder gegabelt, ganz. Sp. G-H. Orn. 3, Grösse $8 \times 6.5$.

R. emetica Schff. - 24.7. - Sammelart.

$R$. gracillima J. Schaeffer - M. 30.8. - H. 4 breit, in der Mitte olivgrau, am Rande rötlich, gerieftgefurcht. Haut bis zur Mitte leicht abziehbar. F. $5 \times 7.5$, weiss, rot angelaufen, keulig, schwammig, gebrechlich. L. weiss, dünn, gebrechlich. Sp. G-D. Orn, 6, Grösse $8 \times 7$. Etwas scharf. Am Hofe.

R. sanguinea Bull. -24.7 .

R. exalbicans Secr. - M. 16.7. - H. bis 8 breit, hellziegelrot bis beinahe weiss, mit roten Flekken (ausbleichend). Nur wenig abziehbare Haut. Stumpfer, glatter Rand. F. weiss, unten verdickt, bis $8 \times 20$, innen schwammig. L. crème bis hellocker, angewachsen, bisweilen gegabelt. Sp. E-F. Orn. 4. Grösse $8-8.5 \times 6.5-7$. L. scharf Obstgeruch. Unter Birken.

Lactarius vellereus Fr. - 1.9.

L. controversus Pers. -15.8 .

L. piperatus Scop. - 18.8 .

L. torminosus Schff. -4.7 .

L. repraesentaneus Britz. - 17.7 .

L. uvidus Fr. - 16.8 .

L. deliciosus L. -15.7

L. necator Pers. - 12.8 .

L. vietus $\mathrm{Fr} .-27.8$.

L. trivialis $\mathrm{Fr} .-21.7$.

L. fuliginosus Fr. -15.8 .

L. glyciosmus Fr. -7.7 .

L. flexuosus Fr. -9.8 .

L. lilacinus Lasch -14.8 .

L. mitissimus Fr. -8.8 .

L. obscuratus Lasch ss. Pears. (cyathula Rick.) - M. 14.8. - H. bis 2 breit, olivbraun, mit kleinem verschwindendem Höcker. Rand etwas gerieft, schliesslich faltig. Milch weiss, mild. F. $3 \times 3$, bleich, wird von unten mit $\mathrm{H}$. gleichfarbig. $\mathrm{L}$. fleischgelb. Sp. sehr gross und grobstachelig. $8.5-10 \times 6.5-8$. Laubwald.

L. helvus Fr. -20.8 .

L. rufus Scop. -17.7 .

L. subdulcis Bull. - 2.7. - Sammelart.

Cantharellus cibarius Fr. - 9.7.

Craterellus cornucopioides L. - 6.9.

Crucibulum vulgare Tul. -27.7 .

Calvatia caelata Bull. - 31.8.

Lycoperdon echinatum Pers. - M. 7.8. - 2 bis $4 \mathrm{~mm}$ lange zusammenneigende, pyramidale St. Sp. stachelig, $5 \times 5$, mit Stiel.

L. umbrinum Pers. - 25.7.

L. gemmatum Batsch -21.7 .

L. piriforme Schff. -14.6 .

Bovista plumbea Pers. - 22.6.

B. nigrescens Pers. -9.6 . 
Tremella encephala Willd. - 5.9.

Calocera viscosa Pers. - 16.8.

C. cornea Batsch - M. 22.7. - 5-10 $\mathrm{mm}$ hoch, keulig-einfach, orange. Pfriemen an der Basis zusammengewachsen. Sp. $8-9 \times 4-4.5$, verbogen, hyalin. A. Laubholzstamm.

Clavaria botrytes Pers. -9.8 .

C. aurea Schff. -27.7 .

C. flava Schff. -10.8 .

C. condensata Fr. var. violacea-tincta - 30.8. - Fk. bis $10 \mathrm{~cm}$ hoch und bis $5 \mathrm{~cm}$ breit, ledergelb bis fleischfarben/violett. Gemeinsamer Strunk kurz oder fehlend. Endästchen gelb, zwei- bis dreizähnig. Sp. fast mandelförmig, häufig einseitig flach, 8-10 $\times 4-5$. Myzel weiss.

C. abietina Pers. - 31.8. - Fk. ockergelb bis rehbraun, innen weiss. Unbedeutender Strunk, weissfilzig. Steife Zweige mit 3 und mehr scharfen, grün werdenden Spitzen. Sp. $7-10 \times 4-5$, ockerfarben.rauhlich. Würziger, starker Geruch und bitterlicher Geschmack.

C. Invalii Cott. et Wakef. -21.7 .

C. flaccida Fr. - 13.8. - Fk. höchstens $5 \mathrm{~cm}$ hoch, ockergelb, flatterig-schlaff. Strunk unbedeutend. Sp. gelb, $5-7 \times 3-4$, rauh. Auf Nadeln und Blättern.

C. coralloides L. - M. 14.8. - Nach Bourdot et Galzin nur eine robuste Form von C.cristata. Fk. weisslich, bis $10 \mathrm{~cm}$ hoch, reich verästelt, gebrechlich. Strunk ziemlich dick. Zweige ungleichlang. Endzweige scharf. Sie erscheinen stumpf nur solange der Pilz unentwickelt ist. Sp. hellocker, $8.5-9 \times 5.5$ mit Öltropfen. Rasernartig im Laubwald. Nachprüfung der Bestimmung wegen der in der Lit. abweichenden Farbe der Sp. erwünscht.

C. muscoides L. (corniculata Schff.) - M. 9.9. - Fk. dottergelb, schwach verästelt, $3 \mathrm{~cm}$ hoch. Strunk gleichfarbig, sehr dünn, nur $2-3$ mal gegabelt. Zweige fast rechtwinkelig abgebogen. Endzweige mondförmig. Sp. $5 \times 5$, glatt, farblos. Viehweide.

C. pistillaris L. -17.8 .

C. ligula Schff. -27.8 .

Stereum hirsutum Willd. - 23.7.

S. purpureum Pers. -7.8 .

Pleurodon auriscalpium L. -24.7.

Dryodon cirrhatum Pers. - 19.7.

Hydnum repandum L. -23.7.

H. rufescens Pers. - 10.9 .

Sarcodon imbricatum L. -9.8 .

S. fragile $\mathrm{Fr} .-\mathrm{P} .18 .8 .-\mathrm{H}$. bis 10 breit, weissgrau, später mit rötlich-braunem Ton, gebrechlich, mit welligem Rand. F. $5 \times 20$, schmutzigbräunend. St. weisslich, pfriemlich, fein, zerbrechlich, lang. Sp. farblos, stachelig, 3-4.5 im Diam. Geschmack unangenehm, aber nicht bitter. Nadelwald.

Calodon aurantiacum A. et S. - 13.9.
C. ferrugineum Fr. -30.8 .

C. scrobiculatum Fr. -9.8 .

C. cyathiforme Schff. -9.8 .

Phylacteria terrestris Ehrh. - 20.8.

Polyporus ovinus Schff. - 21.7.

Melanopus melanopus Pers. - 23.7.

Leucoporus brumalis Pers. - 10.6.

Coriolus pubescens Schum. - 15.7. - Weiss, mit gelblichem, scharfem Rand, gezont, flaumig. R. weiss, kurz, rund. Sp. $4.5-7 \times 2-2.5$. Fl. zuletzt korkig, meist von Larven zerstört. An kranken Laubholzstämmen.

C. velutinus Pers. ss. Rick. - 9.7. - Weissgelblich, dunkler gezont, sammetig-weich, kaum höckeriggebuckelt. Substanz weiss. Sp. $5.5-7 \times 1.5-3$, hyalin. Auf Ästen, häufig dachziegelig. Nach Bourdot et Galzin eine Form, die sich von C. pubescens nur wenig unterscheidet.

C. hirsutus Schrad. - 30.6.

C. zonatus Nees -8.6 .

C. unicolor Bull. (Daedalea u.) - 21.7. - Zottig, blass, im Alter graubraun, muschelförmig, mit welligem, weissem, scharfem Rand. R. sehr unregelmässig, scharf. Sp. $4-5 \times 3$, eiförmig bis oval. Auf Laubholz, dachziegelig.

C. abietinus Dicks. -15.7 . - H. bis 5 breit, ergossen bis hütig abgebogen, filzig, grau, schliesslich grünlich, undeutlich gezont. R. eng, eckig, sehr bald zerschlitzt, violettpurpurn (ausblassend). Sp. $6 \times 3$, einseitig eingebogen. Auf Nadelholz.

Irpex sp. - M. 27.6. - Resupinat. Mit deutlichem F. auf altem Birkenstamm. Keine Sp.

I. sinuosus Fr. - 21.8. - Nicht überwinternd. Weiss, ergossen. Sp. $3-4 \times 2.5-4$ (nach $\mathrm{B}$ ou r d o t et $\mathrm{G}$ a $1 \mathrm{z}$ i n $6-7 \times 2.5-4)$. Auf trockenen Birken- und Ellerästen. Bestimmt von Dir. $\mathrm{N}$ yb e r g.

Lenzites betulina L. -15.6 .

L. tricolor Bull. - M. 11.9. - Von mir 52 als Lenzites sp. bezeichnet. H. 8 breit und bis $3 \mathrm{~cm}$ dick, oben striegelig, rötlich mit blassem Rand. P. rötlich, stellenweise intensiv rot. Sp. $6 \times 3$. FI. rötlich, stellenweise weissgelb, weich. Angenehmer Geruch. Ohne Geschmack. Auf toter Birke.

L. sepiaria Wulf. -14.7 .

Trametes cinnabarina Jacq. - 18.6 .

T. odorata Wulf. -27.7 .

Ungulina fomentaria L. - 17.6 .

U. marginata Fr. - 8.6.

$U$. resinosa Fr. (fuliginosa (Scop.) Pat., Ischnoderma benzoinum Vahl, Karst.) - 20.6.

$U$. betulina Bull. - 10.6 .

Ganoderma applanatum Pers. - 6.7.

Phellinus igniarius L. - 10.6 .

Xanthochrous perennis L. -5.7 .

Poria vulgaris Fr. (Physisporus v.) - M. 13 und 17.7.Resupinat mit freiem Rand, weiss, trocken. Sehr dünnes Subiculum. R. weiss, rund, dicht. 0.5-2 $\mathrm{mm}$ lang. Sp. weisslich, $4-6 \times 3-4$, eiförmig bis subsphärisch. Tote Birkenäste.

\section{ASCOMYCETES.}

Gyromitra esculenta Pers. - 8.6.

G. infula Schff. - 4.9.

Cudonia confusa Bres. - 4.8 .
Vibrissea truncorum Schw. - 9.6. - H. einige $\mathrm{mm}$ breit, gelb oder orange. F. weisslich, zuletzt schwärzlich, $1 \times 1-2$. Sp. $150-200 \times 1$. Auf 
verfaultem Holz. Bestimmt von Prof. V. Kujala.

Leotia gelatinosa Hill. (lubrica Scop.) - M. 31.8. Hütchen oliv. Sonst orange. Sp. $17-23 \times 5.5$, spindelig, bisweilen verbogen.

L. sp. - M. 19.8, - 52 von mir unter Mitrula verzeichnet. Fk. keulig-himbeerartig, $8 \mathrm{~mm}$ hoch und $10 \mathrm{~mm}$ breit, orange. F. $15 \mathrm{~mm}$ lang, nach oben verdickend, blassoliv. Sp. stumpfspindelig, $12-13.5 \times 3$, hyalin. Auf. Blättern.

Spathularia clavata Pers. - 29.7.

Otidea sp. - M. 12.9. - $1.25 \times 1 \mathrm{~cm}$. Gelbbraun.
F. kurz, weiss. Sp. $18 \times 9$, mit einem Tropfen. Nadelwald.

Plicaria badia Pers. - 11.8.

P. repanda Pers. (varia Hedw., Stevensonia Rehm.) 18.6. - Isabellbraune Scheibe. Sp. $16-19 \times$ 8-9, elliptisch, ohne Öltropfen.

P. vesiculosa Bull. - 27.6.

Sacroscypha melastoma Sow. oder dieser Art sehr nahestehend - P. 18.8. - Siehe auch Kap. 5. 2.5 breit. Innen schwarzbaun, nich glänzend. Aus. sen grau, grauborstig. Wohl mit kleinem F. Sp. $20 \times 10$, mit einem grossem Tropfen. Waldweg.

\section{De Insel Attu, im Kirchspiel Pargas, südlich Turku}

Dieses Gebiet hatte früher den Ruf sehr pilzreich zu sein. Heute haben sich die Waldbestände, vornehmlich infolge der Kolonisation nach den Kriegen, sehr vermindert. Von den Laubholzbeständen ist leider nur sehr wenig übrig. Immerhin ist die Insel recht ergiebig. Nach dem Charakter der Pilzflora nimmt Attu eine Art Mittelstellung zwischen dem Festlande und Åland ein. Ich verbrachte daselbst die Zeit zwischen dem 16.6. und 12.7. und zwischen dem 18.7. und 6.9.1953. Es war ein sehr günstiges Pilzjahr.

\section{BASIDIOMYCETES}

Suillus luteus L. - 28.7.

S. flavidus Fr. - 15.8 .

S. granulatus L. -29.7 .

S. bovinus L. - 14.8 .

S. variegatus Sow. -6.8

S. piperatus Bull. - 7.7 .

Leccinum aurantiacum Bull. - 24.6. - Zur früheren Sammelart rufum gehörig.

L. rufescens Secr. (testaceoscabrum Sing.) - 19.8. Zur früheren Sammelart rufum gehörig. Gelborange, mit schwarzen Schüppchen am F. Fl. weinviolett anlaufend.

L. leucophaeum Gilb. - 16.6. - Zur früheren Sammelart scabrum gehörig.

L. leucophaeum Gilb. var. coloripes Sing. - 1.9. Mit gelbem Fl, im F.

L. carpini Schulz et Pears. - 7.7. - H. braun bis braunoliv mit globaren Zellen der Oberfläche. Wird meist rissig-felderig. F1. weissrötlich, dann violett, schliesslich grauschwarz. Die $\mathrm{R}$. ragen über die Haut des H. hinaus.

Boletus edulis Bull. - 7.7.

Tylopilus felleus Bull. - 9.7.

Xerocomus badius Fr. - 11.7 .

$X$. chrysenteron Bull. - 11.7.

X. subtomentosus L. -28.7 .

Paxillus involutus Batsch -26.7 .

$P$, atrotomentosus Batsch -23.7 .

Hygrophoropsis aurantiaca Wulf. - 8.8.

Gomphidius glutinosus Schff. - 10.7.

G. roseus Fr. - 2.8 .

G. viscidus L. -24.7 .
Hygrophorus piceae Kühn. - 21.8. - Sieht H. eburneus sehr ähnlich. $\mathrm{H}$. bis 5 breit, weiss, wenig schleimig. F. trocken, bis 6 lang. Basidien 4-sporig. Sp. $7-8 \times 4.5-5$. Geruchlos.

H. eburneus Bull. - 21.8. - Sp. etwas grösser als bei $H$. piceae. Angenehmer Geruch. In Finnland vermutlich seltener als $H$. piceae.

H. cossus Fr. -19.8 .

H. olivaceoalbus Fr. -23.8 .

H. erubescens Fr. -15.8 .

H. pudorinus Fr. -12.8 ,

H. agathosmus Fr. - 11.8.

H. caprinus Scop. -22.8 .

Camarophyllus niveus Scop. - 30.8 .

C. pratensis Pers. -31.8 .

C. streptopus Fr. -30.8 . - H. bis 5 breit, feucht, hellolivgrau. F. $8 \times 8$, grau, hohl, verbogen. L. ausgebuchtet, sehr breit, bauchig, weiss, ziemlich dünn. Sp. $7 \times 4.5$, elliptisch. Vermutlich = Hygracybe fornicata (Fr.) Sing.

Hygrocybe nitrata Pers. -23.8.

$H$. nigrescens Quel. -21.8.

$H$. conica Scop. - 10.8 .

H. chlorophana Fr. -23.8 .

H. ceracea Wulf. - 31.8. - H. 2 breit, wachs- oder strohgelb. F. gleichfarbig, 3 lang. L. dreieckig, blassgelblich, breit, undicht, adrig verbunden. Sp. 6-7 $\times 3-4$, elliptisch. Auf Weideland.

H. Reai Maire var. insipida Lange - 9.8. - H. bis 2 breit, zuerst rötlich, dann gelb, klebrig. F. $2-3$ $\times 2-3$, hohl, klebrig. L. gelborange. Sp. 5.75$6.5 \times 3.25$. Geschmacklos. Im Freien.

H. cantharellus Schw. -20.8 .

H. miniata $\mathrm{Fr} .-23.8$. 
Asterophora lycoperdoides Bull. - 12.8.

Lyophyllum cessans Karst. (Omphalia reclinis Fr.?) 17.8. - Sieht Omph. striaepilea Fr. ähnlich, nur kleiner. Starr. L. schwach herablaufend, beinahe angewachsen. Sp. 5.25-6×4-5. Auf Ästchen und Nadeln.

L. decastes Fr. (aggregatum Schff.) - 2.8. - Lange unterscheidet var. sphaerospora und orispora. $\mathrm{H}$. 5-10 breit, kastanien- oder ockerbraun, ziemlich glatt, nur schwach radial gefascrt. F. weisslich, $5-12 \times 10-20$, unten häufig verbogen. L. weisslich, schwach herablaufend. Sp. $5 \times 5$, aber auch $6 \times 4$ etc. Büschelig zusammengewachsen, aber auch einzeln.

Calocybe Georgii Clus. - 17.6.

C. persicolor Fr. - 21.6. - H. 3 breit, dauernd fleischrosa, kahl, trocken. F. rosa, $3 \times 4$, voll, unten weiss-striegelig. L. cr me, gedrängt. Sp. $5 \times 2.75$. Büschelig, am Wege.

Clitocybe geotropa Bull. var. subinvoluta - 23.6. - Sehr fragliche Art. Lange unterscheidet 2 Varianten, je nach dem ob die Sp. mit oder ohne Schaft sind. Es finden sich aber Ex. die Sp. beider Arten haben. Sp. $6 \times 4.5$.

C. odora Bull. - 1.8.

C. odora Bull. var. alba Lange - 14.8.

C. clavipes Pers. - 21.8.

C. lentiginosa Fr. - 1.9. - Nicht hygrophan. H. 4 breit, fleischfuchsig, beinahe trichterförmig, mit gekerbtem, eingebogenem Rand. F. 5 lang, Farbe des $H$. nach unten verschmälernd, hohl, schliesslich zusammengedrückt. L. gelblich. Sp. rauh, $6 \times 4.25$.

C. inversa Scop. (flaccida Sow.) -27.8 .

C. gilva Pers. -23.8 . H. blass gelbbraun. Sp. stachelig, länger als bei C. inversa.

C. infundibuliformis Schff. -5.8 .

C. sinopica Fr. - 19.6.

C. sinopica Fr. var. sinopicoides Peck - 27.8. - Von mir zuerst als Collybia sp. bezeichnet. Sieht auf den ersten Blick wie C. sinopica aus. H. bis 4 breit, rosenrot, ungerieft, unter Lupe sammetig, flatterig-wogig. F. bis $4 \times 4$, gelblich bis rosenrötlich, gepudert, nicht gerieft, sehr krumm, unten weissfilzig. L. weisslich mit gelblichem Ton, angewachsen, resp. schwach herablaufend. Sp. 5-6x $2.5-3$, oval. Viele Ex. mit Füssen zusammengewachsen, im Freien. Eine Nachprüfung der Bestimmung an der Hand der sehr guten Exsikkate wäre sehr erwünscht. Ich suchte den Pilz auch in der Nähe der Collybia acervata. Dagegen sprechen aber das rasige Wachstum, das Habitat und vor allen Dingen der uncollybiale Charakter der L.

C. phyllophila Fr. -25.8 .

C. pithyophila Secr. -25.8 .

C. cerussata Fr. -28.8 .

C. rivulosa Pers. -23.8 .

C. tuba Fr. -31.7 .

C. candicans Pers. -24.8 .

C. hydrogramma Bull. - 8.8. - H. bis 5 breit, grau, tiefgenabelt, trocken, blass mit bald abstehendem, gerieftem, krausem Rand. F. 2 lang, häufig breitgedrückt, mit weissfilziger Basis. L. gleichfarbig. Sp. $5-6 \times 3-3.25$. Stinkt.

C. diatreta Fr. -25.8 .

C. dicolor Pers. -27.8 .

C. brumalis Fr. -30.8 .
Laccaria laccata Scop. - 25.6.

Tricholomopsis platyphylla Pers. - 16.6.

T. rutilans Schff. -23.6 .

T. decora Fr. - 26.8. - H. 10 breit, gelb, durch angewachsene, schwärzliche Schuppen rauh. F. etwas excentrisch, $10 \times 10-15$, gelb, faserig gestreift. L. gelb, gedrängt, ausgebuchtet angewachsen. Sp. $5.5-6 \times 3$, oval. Fl. gelblich. Mehlgeschmack. An Nadelholz.

Collybia cirrhata Schum. var. typica Maire - 8.8. H. bis 1.5 breit, weisslich mit bräunlichem Ton, seidig. F. rötlich blass, $5 \times 1$. L. weiss, dicht. Sp. $3.25-5 \times 2-3$. Ohne Sclerotium, auf Resten fauler Pilze.

C. tuberosa Bull. - 22.8. - H. bis 1 breit, weiss. F. weisslich mit bräunlichem Ton, $2 \times 0.5$. L. weiss. Sp. $4 \times 3$. Braunes, dann schwarzes Sclerotium. Auf Resten fauler Pilze.

C. peronata Bolt. -29.7 .

C. confluens Pers. -22.6 .

C. macilenta Fr. -4.8 . - H. bis 3 breit, dunkelocker, ungerieft. F. $3 \times 4$, gerieft, gelblich, unten weissfilzig. L. dunkelocker, gedrängt, angewachscn. Sp. $4-4.5 \times 2.75-3$, oval. Auf Nadeln.

C. ocellata Fr. - 5.8. - H. 1 breit, weiss, in der Mitte mit niedergedrücktem, etwas dunklerem Scheibchen. F. $3 \times 1-2$, glatt, gelbweisslich, unten etwas bräunend, zäh, wurzelnd. L. weiss, schmal, ziemlich gedrängt, ganze und halbe wechselnd. Sp. $7.5 \times 4.5$, eiförmig. Im Grase.

C. nummularia Fr. - 31.7. - Hygrohan. H. bis 3 breit, blass, wird beinahe weiss. Mitte gelblich. Sonst wie G.aquosa. Nadelwald.

C. xanthopus Fr. - 21.6. - Hygrophan. H. einige $\mathrm{cm}$ breit, crèmefarben, trocken beinahe weiss. F. goldgelb, $9 \times 4$, wurzelnd, unten striegelig. Sonst wie C. dryophila.

C. dryophila Bull. - 16.6.

C. dryophila Bull. var. - 19.6. - H. beinahe schwarz braun. Stark hygrophan. Wenn ausgeblasst violettlich-fleischfarben. F. gelbrot, unten dunkelrot.

C. aquosa Bull. - 21.8.

C. butyracea Bull. - 17.8 .

C. maculata A. et S. -30.8 .

C. distorta Fr. -20.8 . - H. bis 6 breit, kastanienrot, glatt, kahl, zuerst gebuckelt, dann geschweift. F. gelblichblass, gerieft, fast gleichdick oder aufwärts verjüngt, verdreht, bis $9 \times 8$. L. weiss, sehr schmal, gedrängt, nicht fleckig, aber bisweilen gekerbt. Sp. 3-4 in Diam. Geruchlos. Mischwald.

C. tesquorum Fr. - 16.8. - Nur ein Ex. H. 4 breit, dunkel graubraun, nackt. F. $5 \times 5$, heller als $\mathrm{H}$., faserig gestreift. L. sehr dicht, weisslich. Sp. stache. -lig-warzig, beinahe wie bei Russulae, $7-8 \times 5$ -6Auf der Insel Vånå.

Omphalina sp. - 27.7. - H. bis 3.5 breit, gelblich-graubraun, mit dunkleren radialen Fasern besetzt, tief genabelt, schliesslich längs einigen Fasern aufspaltend. F. bis $10 \times 5$, oben gelblich, unten dunkler, kahl, faserig gestreift, bisweilen plattgedrückt, schief wurzelnd. L. weit herablaufend, gelb, breit, adrig, bisweilen mit einander verbunden. Der $\mathrm{H}$. besteht eigentlich nur aus Haut und L. Sp. 6-7 $\times 4.5-5$, oval, weiss. In der Nähe eines Stumpfes, aber nicht auf diesem.

O. scyphiformis Fr. ss. Langei - 22.7. - H. 1 breit, weiss, zuerst konvex mit tiefem Nabel und einge- 
bogenem Rand, dann ausgebreitet. Rand wenn trocken gefurcht-gerieft. F. $3 \times 1$. Basis weissstriegelig. L. etwas herablaufend, mitteldicht. Sp. oval, $6-7 \times 4-4.5$, mit oder ohne Öltropfen. Einzeln im Walde.

O. oniscus Fr. - 7.8.-Omph. philonotis Lasch nahestehend. F. 1 breit, graubraun, schwach durchscheinend gerieft, tief- und spitzgenabelt, fast durchbohrt. F. graubraun, $2 \times 2$, zusammengedrückt. L. ziemlich undicht. grau. Sp. $7 \times$ $4.5-5$, eiförmig bis oval.

Armillariclla mellea Vahl - 16.8.

Tricholoma flavobrunneum Fr. - 6.8.

T. vaccinum Pers. -28.7

T. imbricatum Fr. -30.8 .

T. equestre L. -6.9 .

T. inamoenum $\mathrm{Fr} .-30.7$

T. album Schff. -29.8 .

T. saponaceum Fr. -21.8 .

T. virgatum $\mathrm{Fr} .-1.8$.

Cantharellula umbonata Gmel. - 26.8.

Melanoleuca strictipes Karst. - 22.6.

M. melaleuca Pers. - 8.8.

Pleurotus cornucopiae (Pers. ex. Paul.) Sacc. (sapidus Schulz et Kalchbr.) - 26.7. - H. bis 15 breit, weissgrau bis lederfarben, trichterförmig. Deutlicher F., bis 6 lang, excentrisch. L. tief herablaufend, auf dem F. anastomosierend. Sp. gewöhnlich $9 \times 4$, farblos mit rosa Schimmer. Rasig, auf einem totem Laubholzstammc.

Panus conchatus Fr. - 26.6.

Lentinus lepideus Fr. - 24.6.

Hohenbuehelia petaloides Bull. - 1.9. - H. bis 10 breit, nierenförmig, violettgrau, kahl. F. ganz kurz, aber deutlich, weisslich, sehr excentrisch, aber mit ringsum gerandetem. H. L. weit herablaufend, sehr dicht, beinahe Farbe des H. Sp. warzig. $5.5-6 \times 4.5-5$. Im Nadelwalde, vermutlich auf Wurzeln. - Gehört jedenfalls der schlecht untersuchten Gruppe von P. geogenius an.

Micromphale perforans Hoffm. - 17.7.

Pseudohiatula tenacella (Fr.) Métrod ss. Tuomik. (Marasmius conigenus ssp. esculentus Wulf. ss. Favre) - 17.6. - Die alte Sammelart Coll. esculenta-tenacella ist nunmehr in drei Unterarten zerlegt worden, von denen diese eine ist. Sp. $5-7 \times 2.8-4$. Stumpfe Cyst. $40-80 \times 10-20$. Auf Fichtenzapfen. Ich habe die anderen Unterarten vermutlich auch gesehen, ohne sie näher zu untersuchen.

Marasmius scorodonius Fr. - 20.7.

M. ramealis Bull. -4.8 . - $\mathrm{H}$. bis 1 breit, ungerieft, runzelig, weisslich, Mitte mit dunklerem bräunlichem Ton. F. bis $2 \times 0.5$, oben hell, unten rotbraun, mehlig. L. weiss, kaum collybial, eher etwas herablaufend, nicht sehr dicht, schmal, untermischt. Sp. 8-9 $8 \times 3-3.25$, lancett- bis kernförmig. Auf Holzstücken.

$M$. oreades Bolt. -16.6 .

M. Wynnei Bk. et Br. (globularis Fr.) - 21.6. - H. einige $\mathrm{cm}$ breit, zuerst halbkugelig, weiss-crème, wird grauviolettlich. F. $5 \times 2$, hornartig, bestäubt, weiss, von unten bräunend. L. crème, dann violettlich, breit, entfernt, frei, queradrig. Sp. 6$7 \times 4$. Wegrand.

M. Wettsteinii Sacc. et Sydow (tenerrimus Wettst. non Berk., Nybergii OvS.) - 21.6. - H. bis 0.4 breit, weiss, dunkel genabelt-gefurcht. F. $2-5 \times 0.25-$ 0.33 , oben hell, unten dunkel. Gewöhnlich $15-16$
L., die zu einem freien Halsring hinten verbunden sind. Sp. gewöhnlich $7.5 \times 4.5$. Auf Nadeln. M. androsaceus L. - 17.6.

Xeromphalina campanella Batsch -20.6 .

Mycena haematopoda Pers. - 29.8.

M. galopoda Pers. var. nigra -4.8 .

M. vitilis Fr. ss. Schroeter, Rick. - 7.7.

M. galericulata Scop. -13.7 .

$M$. rugosa Fr. -26.6 .

M. inclinata Fr. - 29.8, - H. bis 3 breit, bräunlich, gerieft, zuerst kugelig mit gezähncltem Rand, dann glockig. F. $7 \times 2$, oben weissgelb, unten dunkelrotbraun. Basis weiss-striegelig. L. blass, breit, ziemlich dicht. Sp. $8.5 \times 6$. Cyst. breitkeulig mit Haaren. Rasig auf Eichenstumpf.

M. epipterygia Scop. -22.8 .

M. rubromarginata Fr. - 7.8. - H. bis 2 breit, braunrötlich. Mitte dunkler, schwach gerieft. F. gleichfarbig (etwas heller), unten dunkler und behaart. L. blass mit roter Schneide. Sp. 9-11x 5.5-7. Gyst. spitz, schmale Spulenform,

M. rosella Fr. -22.8 .

M. pura Pers. - 18.6 .

M. laevigata Lasch - 8.8. - H. bis 2 breit, weiss, gelbfleckig. Rand schwach gerieft, etwas schlüpfrig. F. schlüpfrig, glatt, Farbe des H., wurzelnd, unten mit bläulichem Ton und striegelig. L. weisslich, häufig mit Halsring verbunden. Sp. 6.5$7 \times 4-4.5$. Rasig auf Nadelholz.

M. ammoniaca Fr. -24.6 .

M. aetites Fr. - 12.8. - Vielleicht identisch mit $M$. ammoniaca. Hervorragender stumpfer Buckel. F. sehr lang und dunkel (bräunlich), $12 \times 2$. Sp. $9-10 \times 5-5.5$. Riecht schwach alkalisch.

M. alcalina Fr. - 17.6 .

M. flavoalba Fr. -2.8 .

M. fibula Bull. - 22.8 .

M. lineata Bull. ss. Karst., Rick. - 27.8. - H. $0.5-1.5$ breit, gelb, bis zum Scheitel gefurcht. F. fädig, unten weissfilzig, bis 5 lang. L. ziemlich bauchig. weisslich. Sp. $6-7 \times 5-5.5$. Vielleicht identisch mit $M$. citrinomarginata Gill.

Baeospora myosura Fr. -12.8 .

Clitopilopsis hirneola Fr. (Clitocybe h.) -25.8 . - H. bis 2.5 breit, honiggrau, seidig glänzend. Schliesslich trichterförmig. Dünner, eingebogener, ungeriefter Rand. F. $4 \times 3$, grau, oben weissbereift. L. herablaufend, grau, dünn. Sp. graulich, $5 \times 4$.

Clitopilus prunulus Scop. - 19.7.

Rhodophyllus clypeatus L. - 19.7.

R. nidorosus Fr. -15.8 .

R. rhodopolius Fr. -12.8 .

$R$. cordae Karst. - 27.8. - H. bis 4 breit, dunkelolivbraun, ausgesprochen gerieft am Rande, sehr gebrechlich und dünn. F. $7 \times 6$, faserig gestreift, blassgrau, hohl. Basis weiss und verdickt. L. braungrau, breit. Sp. $7 \times 6$. Ohne Geruch und Geschmack. Im Nadelwalde.

$R$. staurosporus Lange -9.8 .

$R$. hirtipes Schum., Lange -8.7 .

$R$. mammosus Fr. Quel. ss. Konr. et Maubl. (hebes Romagn.?) - 31.7. - H. bis 4 breit, mit meist spitzem Buckel, gerieft, braun. F. blasser, faserig gestreift, bis $9 \times 4$, steif. L. braun, sehr breit. $\mathrm{Sp}$. sehr versch., bisweilen sternförmig, $7 \times 7$, $9 \times 6$ etc. Schwacher Mehlgeruch und Mehlgeschmack. 
R. sericeus Bull. - 11.7 .

R. junceus Fr. - 21.6 .

R. infulus Fr. - 12.7. - H. 1.5-2 breit, rehbraun, gerieft, gebuckelt. F. etwas blasser, $5 \times 3$, sehr starr, L. weiss, dann rosa, sehr gedrängt. Sp. meist oval, mit sehr hervorstehenden Warzen, $8-9 \times 6$. Der ganze Pilz sehr zäh. Nadelwald.

$R$. anatinus Lasch - 1.8. - H. bis 5 breit, braungrau, mit dunkleren Schuppen. Scheitel faserigschuppig oder riefelig-runzelig. F. bläulich, faserig, oben kahl. I. rötlich. Sp. $10 \times 7$ etc. länglich. Viehweide.

R. asprellus $\mathrm{Fr} .-30.7$.

$R$. serrulatus Pers. -2.8 .

R. lampropus Fr. -20.7 .

$R$. rusticoides (Gill) Lange - 23.6. - H. bis 1.5 breit, stark konvex, nur schwach genabelt, zart flockig-filzig, braun. Rand gerieft. F. $2 \times 1-2$, Farbe des H. L. braun bis rotbraun, ziemlich entfernt, breit, hera blaufend. Sp. subrotund, unregelmässig, schwach kantig, 6-7 im Diam. Auch längliche kommen vor, z.B. $7.5 \times 6$. Rötlichgelb. Waldweg.

Pluteus cervinus Schff. - 16.6.

P. umbrosus (Pers.) Fr. ss. Rick. - 12.7. - H. bis 7 breit, schwarz, striegelig-runzelig. F. dunkel, besonders unten. L. mit russfarbener Schneide. Sp. $5.5-6 \times 4-5$.

Volvariella speciosa Fr. -22.6 .

Amanita strangulata $\mathrm{Fr} .-12.8$.

A. vaginata Bull. var. alba Fr. -17.8

A. vaginata Bull. var. (sahnefarben) -7.7 .

A. vaginata Bull. var. fulva Schff. -30.7 .

A. vaginata Bull. var. badia Schff. -9.7 .

A. vaginata Bull. var. plumbea Schff. -9.7 .

A. muscaria L. -22.7 .

A. regalis $\mathrm{Fr} .-7.7$.

A. pantherina DC. -15.8 .

A. virosa Fr. -27.7 .

A. mappa Batsch - 21.8.

A. porphyria A. et S. -23.7

A. rubescens Pers. -22.6

Macrolepiota procera Scop. - 11.8.

M. rhacodes Vitt. - 11.8 .

Lepiota naucina Fr. - 28.7.

L. alba Bres. - 4.9.

L. laevigata Lange (oreadiformis Vel., pratensis Rea) 12.8. - H. bis 3.5 breit, blass, Mitte dunkler, gebuckelt, ziemlich glatt. F. $3 \times 3$, weiss, spärlich wollig-schuppig. Ring schnell verschwindend. Sp. spindelig-elliptisch, c. $12 \times 5$. Schwacher, süsslicher Geruch. Offene Stellen.

L. clypeolaria Bull. - 29.7 .

L. felina Quel. - 25.8. - H. bis 2.5 breit, wenn ausgewachsen mit schwarzer Mitte, zum Rande zu mit schwärzlichen Schüppchen auf weisslichem Grund. F. $8 \times 2$, zur Basis zu schmutzig-grau, mit dunkleren Schüppchen, unten verdickt. Ring unten dunkelgrau. Sp. $7 \times 4$, eiförmig bis elliptisch. Fichtenwald.

Agaricus bisporus Lange - 31.7.

A. haemorrhoidarius Fr. ss. Langei (Langei Moeller) 5.8. - Nur ein Ex. H. 6 breit, bräunlich geschuppt. F. $3 \times 8$. L. rosapurpurn. Sp. $8-9 \times$ $4.5-5$. Fl. rötend.

A. campestris L. -28.7 .

A. arvensis Schff. -26.7
A. silvicolus Vitt. -30.6 .

A. rubellus Gill. - 29.7.

A. rusiophyllus Lasch - 15.8 .

A. brunneolus Lange [vielleicht fulveolus (Lasch.) Pers.] - 30.7. - Nur ein Ex. H. 3 breit, braunschuppig, dickfleischig. F. $3 \times 8$, unten etwas verdickt, bei Berührung gilbend. Dünner, weisser Ring. Sp. ziemlich hell, mit Öltropfen, etwa $5 \times 3.5$. Feldrand.

Cystoderma carcharias Pers. - 26.8

C. amianthinum Scop. -13.8 .

C. granulosum Batsch - 11.8.

C. cinnabarinum A. et S. -16.8 .

Crepidotus variabilis Pers. var. subsphaerosporus Lange - 19.8. - Sp. 5-6×3.25-4.

Ripartites tricholoma A. et S. - 24.8. - H. bis 3 breit, weisslich-lederfarben, angedrückt weisshaarig, mit haarigem Rand, schmierig. F. $3 \times 4$, blass, abwärts verjüngt. L. dunkler als $H$., herablaufend, dicht, schmal. Sp. $5 \times 4$, schwach warzig.

$R$. helomorphus Fr. - 27.8. - Wie $R$. tricholoma. Isabellfarben, nagelförmig, mit kahlem Rand. Sp. $3.75-4$, beinahe global, aber etwas kantigwarzig, schmutzig ocker.

Tubaria pallidispora Lange - 22.7. - Vermutlich nicht identisch mit $T$. pallidispora Lange ss. Kühn. et Romagn. Siehe Nachtrag c). H. bis 1 breit, konvex, fleischrot, mit Schleierresten, kaum hygrophan. F. $3 \times 1$, rostfarben, unten weissfilzig. L. dreieckig, mit Zahn angewachsen, ziemlich dicht, Farbe des H. Sp. subcitronförmig, $9-10.5 \times$ 5.5-6, mit spitzer Basis und Öltropfen, hellgelb. Im Moose des Nadelwaldes. Steht $T$. conspersa Fr. nahe.

T. furfuracea Pers. - 3.8. - Sammelart.

Rozites caberata Pers. - 23.7.

Hebeloma mesophaeum Fr. - 18.8.

H. fastibile Fr. -28.6 .

H. punctatum Fr. -31.8 . $-\mathrm{H}$. bis 4 breit, falb mit rotbrauner, schleimiger, punktierter Scheibe und Schleierresten am Rande. F. $4 \times 7$, blass, hohl. gleichdick, oben gepudert. L. dicht, schmal (2 $\mathrm{mm}$ ), bleich, dann kastanienbraun. Sp. gewöhnlich $11 \times 6$. Kein Rettichgeruch. Nicht bitter. Am Wege.

H. testaceum Batsch - 26.7.

H. pumilum Lange - 5.9. - H. bis 2.5 breit, schmutzig rötlichbraun. Rand heller. Konvex, zuerst beinahe halbkugelig, glatt, etwas klebrig. F. $3 \times 4$, blass, oben flockig, unter dem vergänglichen Schleier faserig. L. breit, ausgebuchtet angewachsen, mit weisslichem Rand (dicht besetzt mit Cyst.). Sp. $8-9 \times 5-5.5$, punktiert. Cyst. bis $30 \times 4-5$. Bitterlich. Geruch schwach Rettich. Weg.

H. crustuliniforme Bull. - 9.8 .

H. longicaudum Pers. - 7.7.

H. pusillum Lange - 20.7. - Exs. 1953 unter $H$. sp. beschrieben. Siehe Nachtrag c).

Inocybe obscura Pers. - 18.6.

I. pusio Karst. -4.8 .

I. geophylla Sow. -5.8 .

I. geophylla Sow. var. lilacina - 18.8 .

I. perlata Cooke - 25.8. - H. bis 6 breit. Scheitel cigarrbraun, zum Rande zu blasser, strahligrissig. F. bis $9 \times 15$, zuerst ganz weiss, dann stark bräunend, faserig gestreift. L. zuerst weisslich, 
dann mit bräunlichem Ton, aber weissem Rand (sterile Zellen). Sp. 8-10 (-11) ×5-6.75(-7), gewöhnlich $9 \times 6$, ei- resp. bohnenförmig.

I. fastigiata Schff. -6.7 .

I. Cookei Bres. - 1.9.

I. descissa Fr. var. auricoma Batsch -18.8 . $-\mathrm{H}$. schmutzig blassocker. F. weisslich. Sp. $9 \times 5$. Cyyst. stumpf, $53 \times 12$. Paracyst. bis 15 breit.

I. descissa Fr. var. brunneoatra Lange - 18.8. - Dunkle Fäserchen auf dem $\mathrm{H}$.

I. abjecta Karst. - 29.8. - H. 1-2 breit, cigarrbraun, zuerst bedeckt mit weissen Fäserchen, später schuppig. F. $2-3 \times 2$, bräunlich, oben weisspuderig. Sp. $8-9.25 \times 4.5-5.5$, spitze Eiform. Cyst. flaschenförmig, 12 breit. Fl. weiss. Geophyllageruch. Vielleicht nur eine kleine Form v. I. flociulosa.

I. lucifuga $\mathrm{Fr}$. -16.8 .

I. posterula Britz. ss. Sacc. - 19.8 .

I. lacera $\mathrm{Fr} .-10.8$.

I. Langei Heim - 20.8. - H. $2-3$ breit, konvex, stumpf gebuckelt, ockerfarben, in Schuppen aufbrechend, besonders am Rande. F. weiss, bis $3 \times 3-4$, cylindrisch, feinfaserig gestreift, oben flockig. L. zimtgelb mit Olivton. Sp. $7.5 \times 5$, eiförmig bis elliptisch. Cyst. kurz, breit, bauchig. Waldweg.

I. asterospora Quel. - 1.8.

I. praetervisa Quel. - 22.6.

I. napipes Lange - 26.7 .

I. umboninota Peck -20.7 .

I. umbrina Bres. - 7.8. - H. bis 2 breit, dunkelbraun, schwach gebuckelt. F. $3 \times 3$, mit fast gerandeter Knolle, faserig gestreift, Farbe des H., aber heller, oben schwach kleiig. L. sehr schmutzig. Sp. $8-9 \times 5-6$, mit stumpfen Warzen.

I. Boltoni Heim - 5.8 .

I. lanuginella Schroet. ss. Langei -3.8 .

I. putilla Bres. - 14.8. - H. bis 1.5 breit, konisch spitz, dann ausgebreitet mit Buckel, schliesslich strahlig-rissig, rehbraun. F. $3 \times 1.5$, bleich, ohne Knolle. L.-Rand unter Mikroskop gezackt. Sp. $9 \times 6$, ohne stark hervortretende Warzen. Cyst. flaschenförmig, bisweilen sehr breit. Geophyllageruch.

I. lanuginosa Bull. - 16.6.

I. lanuginosa Bull. f. typica Heim. - 22.6. - Mit aufgeblasenen, flaschenartigen Cyst.

Galerina hypnorum Schrank. - 3.9.

G. mycenopsis Fr. - 2.9. - Einfarbig ockergelb. H. 1-2 breit, bis zur Mitte zart gerieft, anfangs mit weiss-seidig beschleiertem Rand. F. zuerst weiss-seidig gestiefelt, $6 \times 2$. L. bauchig, breit angewachsen. Sp. $10-15 \times 5-5.5$, eiförmig, dickwandig, völlig glatt. Cyst. lang und 5-7 breit. Rettichgeschmack. Auf Moos.

G. marginata Batsch -5.9 .

G. tenuissima Weinm, -20.8 . - H. bis 0.5 breit, gerieft, blassoliv, trocken dunkeloliv. F. zimtgelb, einige $\mathrm{cm}$ lang, fadendünn. L. zimtfarben, dicht. Sp. $7.5 \times 4.5$, hell, eiförmig.

Gymnopilus picreus Fr. - 24.8.

G. liquiritiae Pers. - 19.7. - H. kahl, hygrophan, dünnfleischig. F. ohne Schleier, ohne Reif, wird dunkelrostfuchsig. Fl, gelb.

G. penetrans Fr. - 8.7.

Dermocybe cinnamomea L, var. lutescens Hry - 17.6.

D. malicoria Fr. -10.8 .

D. crocea Schff. -7.7 .
D. semisanguinea Fr. -19.7 .

D. sanguinea Wulf. -10.8 .

Cortinarius traganus Fr. -29.7 .

C. alboviolaceus Pers. -21.8 .

C. camphoratus Fr. - 12.8 .

C. violaceocinereus Pers. -6.8 .

C. opimus Fr. ss. Rick. -23.8 .

C. Bulliardi (Pers.) Fr. ss. Moseri - 30.7. - H. bis 5 breit, dunkel rotbraun, glatt. F. $10 \times 15$, keulenförmig, mit roten Fasern bedeckt (besonders unten). L. Farbe des H., breit, dicht, besetzt mit Cyst. Sp. $9-12 \times 5-7$, oval bis subcitronförmig, rauhwarzig. Fl. läuft citrongelb an. Nadelwald.

C. anomalus Fr. - 10.8.

C. raphanoides Pers. -8.8 .

C. gentilis Fr. - 11.8 .

C. callisteus Fr. - 11.8. - Ein auffallender, effektvoller Pilz, mit leuchtender Farbe. H. bis 6 breit, lebhaft fuchsig-gelb, nach dem Rande hin in kleine, gedrängte, sparrige Schüppchen aufgelöst. F. $12 \times 10$ (oben), faserig gestreift, gold- bis rostgelb. Unten mässige Knolle. L. etwa wie der H. gefärbt. Sp. $7-9 \times 6-8$. Fl. überall gelb, nach unten $\mathrm{zu}$ im $\mathrm{F}$. immer dunkler werdend (Rhabarber). Geruchlos.

Hydrocybe armillata Fr. - 21.8

H. colus Fr. ss. Rick, - 7.7. - H. bis 7 breit, dunkel braunrot, ausblassend, flach gebuckelt. F. $10 \times 10-15$, mit umbedeutender Knolle, oben gelbblass, unten cinnoberrot, faserig gestreift. L. breit, ausgebuchtet, mit Zahn angewachsen, zuerst rot dann schmutzigkanel mit heller Schneide Sp. 6.25-7.25 ×4.5, mandelförmig bis oval, rauh. Nasser Nadelwald.

H. helvelloides Fr. - 11.8. - H. 2 breit, stumpf gebuckelt, gelbzimt, faserig gerieft. F. $8 \times 2$, mehrfach gelbgestiefelt, verbogen. L. sehr dick und entfernt, werden zimtfarben. Sp. $9-10 \times 6$, mandelförmig. Fl. rostgelb.

$H$. saniosa Fr. ss. Langei et Moseri -2.8 .

H. bicolor Cooke. - 9.7. - H. 2-4 breit, violettbis schmutzigbraun mit dunklerem Buckel, trocken sehr blass. F. bis $8 \times 7$, weissbläulich, cylindrisch. Fl. im F. blau. L. blasskanel mit violettlichem Ton. Sp. $9 \times 5.5$, rauh. Rettichgeruch. Nasser Nadelwald.

H. torva Fr. - 12.8 .

H. castanea Bull. - 25.6. - Relativ klein und zäh. $\mathrm{H}$. bis 5 breit, dunkel kastanienbraun. Trocken nur wenig entfärbend. F. $4 \times 8$, schmutzigbraun, oben violettlich. L. anfangs mit violett Ton, dann sehr dunkel rostfarben. Sp. $7.5-8.5 \times 5$, eiförmig oder elliptisch.

H. erythrina Fr. - 27.6. - Sammelart.

H. bulbosa Sow. -4.9 .

H. laniger Fr. - 1.9.

H. bivela $\mathrm{Fr} .-2.9$.

$H$. triformis $\mathrm{Fr} .-5.9$.

$H$. subferruginea Batsch -8.8 .

H. biformis Fr. - 11.8 .

H. armeniaca Schff. -16.8 .

H. diluta Pers. ss. Rick. -14.8 .

$H$. diluta Pers. ss. Langei, non Rick. - 15.8. - H. 3 breit, glanzlos, graubraun mit verschwindendem Buckel, trocken dunkel lederfarben. Am Rande zuerst weissflockig. F. $4 \times 4$, Basis 6 breit, blass. L. hellkanel, ausgebuchtet, bauchig. Sp. $10-11 \times$ 6 , schwach punktiert, mandelförmig bis oval. 
H. rigens Pers. -8.8 .

H. duracina Fr. - 17.6.

H. hinnulea Fr. -31.8 .

H. glandicolor Fr. -13.8 .

H. brunnea Fr. - 11.8.

H. brunneo-fulva Fr. -21.8 .

H. hemitricha Fr. -20.8 .

H. incisa Pers. - 19.8.

H. flexipes Pers. - 19.8 .

H. paleacea Weinm. - 12.8 .

H. rigida Scop. - ss. Karst. - 26.8. - H. 2-4 breit, kastanienbraun. Rand lange weiss durch Schleierreste. F. braun, weiss überfasert, schlank (z.B. $6 \times 4$ ), häufig verbogen. Gürtelzone undeutlich. L. ziemlich dicht und breit, ausgebuchtet angewachsen. Sp. $10-11 \times 5-5.5$, mandelförmig, auffallend hellgelb und glatt.

H. holophaea Lange -22.7 .

H. subbalaustina Hry -25.7 .

H. rubricosa Fr. -29.7 .

H. uracea Fr. ss. Karst. -23.6 .

H. uracea Fr. ss. Langei -30.8 . - H. bis 3 breit, schwarzbraun, wenig hygrophan. mit spitzem Buckel. F. gleichfarbig, bis $10 \times 5$. Basis wejsslich, wurzelnd. L. breit und undicht. Sp. $8-9 \times 5-6$, rauh, punktiert, mandel- bis eiförmig. Fl. sehr dunkel.

H. scandens Fr. -28.7 .

H. obtusa Fr. -8.8 .

$H$. decipiens Fr. - ss. Langei -21.8 .

H. fasciata Fr. -21.6 .

H. Junghuhnii Fr. - 23.6.

H. acuta Fr. -26.6 .

H. sp. - 23.8. - H. bis 2 breit, kastanienbraun mit viol. Ton, trocken hell-fuchsig, ungerieft, hoch -und spitzgebuckelt. F. $3 \times 3$, unten Farbe des H., oben heller. Weisses Velum in der Mitte. L. hellkanel, breit, ausgebuchtet. Sp. $6.5 \times 5-5.75$. Sieht H. decipiens flüchtig ähnich. Myxacium delibutum Fr. - 10.8 .

M. collinitum Fr. - 6.8.

M. mucifluum Fr. - 3.9. - Wie $M$. collinitum. Geriefter Rand. Sp. $13 \times 7$, warzig. Cyst. ballonförmig, $16-20$ breit.

M. elatius Fr. -26.8 .

M. mucosum Bull. - 25.7.

M. vibratile Fr. -3.9 .

M. pluvium Fr. - 25.8. - H. 1-2 breit, ockerfalb, hygrophan. F. $5 \times 2$ (oben) -5 (unten), weisslich, ganz unten zugespitzt. L. ocker, gedrängt. Sp. $7.5 \times 5$, eiförmig. Bitter.

Phlegmacium nemorense Fr. ss. Lange - 23.7.

P. balteatum Fr. - 6.7. - H. bis 10 breit, braun/ lehmfarben. Rand violett, eingebogen. Wenig klebrig. F. kurz, dick, $4-7 \times 20-40$, über dem Schleier weiss-sammtig, unten weiss-zottig gestiefelt und mit rostbraunen Fäden besetzt. L. abgerundet, ziemlich schmal, zuerst bleich, dann lederfarben bis braun. Sp. $9-11 \times 5-6$. Fl. weiss, fest.

P. talum Fr. - 22.8 .

$P$. rapaceum Fr. f. major Lange -15.8 .

$P$. multiforme Fr. im Sinne der älteren deutschen Forscher (Sammelart) - 15.8.

$P$. allutum Secr. - 1.8. - Vermutlich multiforme Fr. var. rufescens Hry. - H. braunrot. Weisse, abgesetzte, eiförmige Knolle. Sp. 8.5-9.5 ×4.55.5 , eiförmig bis elliptisch. Sonst wie $P$. melliolens Schff.
P. multiforme Fr. (ochropallidum Hry) - 21.8. Blass.

P. claricolor Fr. ss. Rick. non Langei - 19.7.

P. turmale Fr. -21.8 . - H. bis 10 breit, ockergelb, schmierig. F. reinweiss, cylindrisch, zottig gestiefelt, fest, sehr hart, bis $12 \times 20$. Schleier herabhängend, dauerhaft. L. zuerst weiss, allmählich lehmfarben, schwach gesägt. Sp. $7.5-9 \times 3.5$ -4.5, mandel- bis beinahe spulenförmig. Fl. reinweiss.

P. triumphans Fr. -21.8 .

$P$. cliduchum Fr. ss. Konr. et Maubl. nec. Rick. (cephalixum Secr. ss. Moseri) - 17.8. - H. bis 6 breit, gelbocker mit Olivton, sehr klebrig. F. $10 \times 10$ (oben). Knolle bis 30 breit. Farbe des F. blass, mit flüchtigen ockerfarbenen oder olivbräunlichen Gürteln. L. lehmfarben, breit, etwas gekerbt. Sp. 9-11 $\times 4.5-6$, mandelförmig, ohne Tropfen, aber mit körningem Inhalt. Ohne auffallenden Geruch.

P. cephalixum Henry, non Secr. - 12.8.

$P$. decolorans Pers. ss. Cke 15.8 .

$P$. decoloratum Fr, ss. Langei var. betuleti Fr. -10.8 .

$P$. orichalceum Batsch -24.8 .

$P$. fulmineum Fr. Aufgefasst genau im Sinne von Fries. - 1.9. - H. 10 breit, feurrotbraun, zum Rande in Gelb übergehend, klebrig. F. $10 \times 15$, mit niedergedrückter, gerandeter Knolle, blass, von unten gelbbräunend, faserig gestreift. L. zimtoliv. Sp. 8-10.5 $\times 5-6.5$, citronförmig, rauh. Fl. weiss, fest, nur in der Knolle und am Rande des $\mathrm{H}$. gelblich.

$P$. infractum Pers. -15.8 .

P. sp. - 28.8. - Exs. mit Bestimmung $» P h$. serarium Fr.» Diese Bestimmung ist falsch. Ich fand den wahren $P$. serarium im Gebiete Tuorila am 26.8.54. Beschreibung des falsch bestimmten Pilzes: H. bis 7 breit, orngefalb, mit faserigem Rand, schmierig, weissbereift. F. c. $8 \times 10$, weiss oft flockig. L. zimtfalb, fast herablaufend. Sp. $8.5-9 \times 5-5.25$. Nachträgliche Bestimmung kaum durchführbar.

P. papulosum Fr. ss. Rick. - 23.8, - H. bis 6 breit, falb mit fuchsigem Scheitel, sehr schleimig. F. bis $8 \times 10$, unten kaum verdickt, faserig gestreift, weiss. L. zimtfalb. Sp. $11-12 \times 6-7$, mandelförmig mit einem grossem Öltropfen. P. papulosum Fr. wird bald mit cliduchum Fr. ss. Konr. et Maubl. bald mit cephalixum Hry identificiert. Mein Pilz kann der erstere sein. Unklarer Fall. Leucocortinarius bulbiger A. et S. -19.8 .

Conocybe tenera Schff. -17.6 .

C. tenera Schff. f. convexa -12.7 .

C. tenera Schff. f. minor -26.7 .

Pholiotina teneroides Lange. Langsporige Form von togularis Bull. (Galera spartea Fr.) - 6.8. - H. $1-2$ breit, zimtbraun, gerieft. Trocken lederfarben und ungerieft. F. blass, $4 \times 2.5$, oben weissbereift. Sp. 9-12 $\times 4.5-6$. Basidien zweisporig. Zwischen Moosen.

P. appendiculata Lange et Kühn. (Galera ravida Fr. ss. Rick.) - 25.8. - H. bis 1.5 breit, lederocker mit weissen Schleierresten am Rande. F. kurz, $1-1.5 \mathrm{~mm}$ breit, oben flockig, Mitte bräunlich, unten filzig. Sp. $7.5 \times 4.5$, ei- bis birnenförmig. Cyst. stumpf, flaschenförmig.

Bolbitius vitellinus Pers. - 22.6.

B. fragilis L. - 19.6 . 
B. titubans Bull. - 29.7.

Agrocybe praecox Pers. - 17.6

Pholiota flammans Fr. -3.8 .

P. tuberculosa Schff. -4.8 .

$P$. carbonaria Fr. -18.6 .

P. spumosa Fr. -7.8 .

$P$. astragalina $\mathrm{Fr}$. -13.8 , - $\mathrm{H}$. bis 6 breit, rotsafrangelb, schwärzt häufig bei Berührung. Rand blass. F. blass, $12 \times 5$, schuppig. L. gelb, später rostfarben. Sp. $6 \times 4$, hellgelb, oval. Fl. rhabarberfarbig. Bitterlich. Auf Nadelholzstämmen.

P. alnicola Fr. -2.9 .

P. scamba Fr. - 26.8. - H. $1-3$ breit, hell lehmfarben, klebrig. F. $2-3 \times 2$, häufig verbogen, oben gelblich, unten lehmfarben. Basis weissfilzig. L. olivbraun. Sp. $8-9 \times 5-5.75$, eiförmig bis oval, durchsichtig, Auf verfaulten Holzstücken im Nadelwalde.

Kuchneromyces mutabilis Schff. - 16.6.

Naematoloma capnoides Fr. - 15.8.

N. sublateritium Fr. -29.8 .

N. fasciculare Huds. -7.8 .

Stropharia depilata Pers. - 31.8.

S. aeruginosa Curt. - 29.8 .

S. coronilla Bull. -27.7 .

S. melasperma Bull. - 16.6 .

S. semiglobata Batsch - 16.6.

Psilocybe merdaria Fr. - 17.6.

P. coprophila Bull. -5.7 .

Deconica atrorufa Schff. - 30.8.

D. bullacea Bull. - 3.9.

Panaeolus campanulatus L. - 27.7

P. sphinctrinus Fr. -2.8 .

$P$. subbalteatus $\mathrm{Bk}$. et $\mathrm{Br}$. -30.7 .

P. fimicola Fr. -21.6 .

Panaeolina foenisecii Pers. - 26.7.

Anellaria separata L. - 17.6.

Psathyrella Candolleana Fr. - 18.6.

P. spadiceo-grisea Schff. - 16.6. - H. bis 5 breit, dattelbraun, trocken lederbraun; bis zur Mitte gerieft, schwach gebuckelt. $\mathrm{F}$. weiss, $8 \times 4$. L. schmutzig-dunkelbraun. Sp. $7.5-5.5$, braun mit violettlichem Ton, eiförmig bis oval. Neben Stämmen.

Pseudocoprinus disseminatus Pers. -5.8 .

Coprinus atramentarius Bull. - 30.7.

C. domesticus Pers. - 31.7.

C. Friesii Quel. - 30.8. - H. bis 2 breit, lederweisslich, mit gelblichem Scheitel und gerieftem Rand. F. bis $7 \times 2$, gewöhnlich $3 \times 2$, weiss, staubig, mit verdickter, etwas behaarter Basis. L. weiss, dann violett-braun. Sp. $7.5 \times 6$, etwas kantig, braun. Auf Gras.

C. cordisporus Gibbs (Patouillardi Quel., papillatus Fr. ex Batsch, angulatus Peck.) - 18.6. - H. einige $\mathrm{mm}$ bis $1.5 \mathrm{~cm}$ breit, grau mit gelblichem Scheitel, gefurcht-gefaltet. $F$. weiss, $3 \times 1$, unten mit weissem Mehl bedeckt. L. frei, schwarz, bestaubt. Sp. citron- resp. herzförmig-runzelig, $6.5-8.5 \times 6-7,8 \times 7 \times 5$ etc. schwarzbraun.

Russula delica Fr. -24.6.

R. densifolia Secr. -20.7

$R$. adusta Pers. -29.7 .

$R$. foetens $\mathrm{Fr} .-1.7$

$R$. sororia Fr. ss. Schaeffer -12.7 .

R. consobrina Fr. -30.6 .
R. lepida Fr. - 3.7. - H. rosenfarben, hartfleischig. F. weiss, bisweilen mit rötlihem Ton, hart. Sp. B, Orn. 4, Grösse 8-9×6-7.

$R$. lepida Fr. var. lactea (Pers.) Fr. - 6.7. - Weisser $\mathrm{H}$.

R. lilacea Quel. - 3.7. - H. bis 7 breit, violettfleichfarben (etwa wie $R$. vesca), klebrig, gerieft. Haut leicht abziehbar. F. weiss oder rötlich anlaufend, weich, gebrechlich. L. weiss, breit, frei. Sp. B, Orn. 6, Grösse 7-9 $\times 6-7$. Mild, geruchlos. An einem Wege im Laubgebüsch.

R. azurea Bres. - 7.8. - H. bis 5 breit, Farbe Gemisch von blau, lila und grau. Stumpfer, wenig geriefter Rand. Abziehbare Haut. F. $3 \times 10-15$, weiss, fest, schwach runzelig. L. dicht, weiss, teilweise gegabelt am F., selten halbiert. Sp. A, Orn. 8, Grösse $8 \times 7$. Nadelwald.

$R$. heterophylla Fr. f. viridis. -8.7 .

$R$. vesca Fr. - 10.7.

R. claroflava Grov. -6.7 .

R. decolorans Fr. - 18.7 .

$R$. vinosa Lindbl. (obscura Rom.) - 12.7.

$R$. xerampelina Schff. var. erythropus (Pelt.) M. et Z. 28.6. - Weinrot mit blutrotem F.

R. xerampelina Schff, var. Barlae Mass. - 9.7.

$R$. xerampelina Schff. var. olivascens (Fr.) M. et Z. - 17.8.

R. xerampelina Schff, var. (gelbrot mit weissem F.) 29.6 .

R. venosa Vel. ss. Melz. -26.6 .

$R$. nitida Pers. ss. Pears. -29.7 .

$R$. puellaris Fr. -7.7 .

$R$. lutea Huds. -15.8 .

R. chamaeleontina Fr. ss. Langei - 23.7.

$R$. aeruginea Lindbl. -27.6 .

R. paludosa Britz -20.7 .

R. Velenovskyi M. et Z. - 20.7. - H. bis 7 breit, ziegelrot. Randschwach oder nicht gerieft. Haut halbabziehbar, klebrig. F. bis $5 \times 15$, weiss, an der Basis häufig einseitig rötlich, cylindrisch. L. crème. Sp. E-F, Orn. 6, Grösse 8-9 6-7. Fl. weiss. Ohne Geruch und Geschmack. Sieht $R$. decolorans ähnlich. Mischwald.

$R$. integra L. -7.8 .

R. alutacea $\mathrm{Fr} .-2.8$.

R. Romellii Maire - 25.7.

R. emetica Schff. - 19.7

$R$. versicolor Schff. -21.7

$R$. sanguinea Bull. - 29.7.

R. Queletii Fr. - 28.7 .

Lactarius torminosus Schff. - 9.8.

L. scrobiculatus Scop. -20.7 .

L. repraesentaneus Britz. -8.8 .

L. uvidus Fr. -24.8 .

L. deliciosus L. -10.7 .

L. necator Pers. -30.7 .

L. vietus Fr. -23.8 .

L. trivialis Fr. -19.7 .

L. picinus Fr. - 11.8. - Ähnlich L. fuliginosus. Viel dunkler, besonders der F. Milch scharf. L. durch Milch gilbend. Sp. $8-9 \times 8$, grobstachelig, mit breiten verbindenden Linien zwischen den Warzen. Fl. langsam rötend. Var. speciosus Lange $=$ Laubwaldform.

L. glyciosmus Fr. - 19.8 .

L. flexuosus Fr. -3.7 .

L. mitissimus Fr. -29.8 .

L. helvus Fr. - 26.7 . 
L. camphorotus Bull. - 28.7. - Milch molkenartig, wässerig, mild. H. bis 5 breit, rot- bis purpurbraun, runzelig, oft mit gekerbtem Rand, meist mit Papille. F. dunkelpurpurn, mit fleischblasser, striegeliger Basis. L. rötlichbraun, herablaufend. Sp. $7.5 \times 7$, grobwarzig, bei starker Vergrösserung netzartig. Geruch etwa von $L$. helvus. Waldweg.

L. rufus Scop. - 29.7 .

L. subdulcis Bull. sensu restr. Langei - 24.6. Milch wässerig, weisslich, erst mild, dann bitterlich. H. bis 5 breit, meist gebuckelt, Farbe heller als L. camphoratus. F. blasser als H., nachdunkelnd, unten zottig. Sp. $7-8 \times 6$ mit meist isolierten Warzen. Cyst. spitz, spulenförmig, 5-6 breit. Ziemlich geruchlos. Dieser wenig charakristische Pilz bleibt übrig, wenn man $L$. camphoratus und theiogalus s. lat. aus der alten Sammelart subdulcis ausscheidet.

L. theiogalus Fr. - 21.7. - Sammelart. Siehe Nach$\operatorname{trag} \mathrm{c})$.

Cantharellus cibarius Fr. - 17.6.

C. infundibuliformis Scop. - 23.8.

C. lutescens Pers. - 10.8.

C. albidus Fr. (Clitocybe a.) - 30.8. - H. 1.5 breit, mit kleinem Buckel, ungerieft, weissgelblich. F. $2 \times 4$, gleichfarbig, verbogen. L. weisslich, gedrängt, herablaufend, teilweise gegabelt. Sp. $5-7 \times 3-4$, oval bis eiförmig. Weideland.

Leptoglossum glaucum Batsch - 30.8. - H. 1 breit, grau, zungenförmig, seidenfaserig. F. seittich, kurz, bereift, grau. L., grau, niedrig, faltenförmig, entfernt. Sp. 6-6.5 $\times 4.5$, eiförmig. Auf Moos. Craterellus cornucopioides L. -2.9 .

C. pallidus Pers. - 9.7. - H. 1.5 breit, trichterförmig, blass, undeutlich gezont, lederzäh, striegelig. F. gleichfarbig. Hymenium feinborstig. Keine Sp. erhalten. Waldweg.

Rhizopogon luteolus Fr. - 1.8. - Weiss, gilbend, schliesslich oliv. Sp. $6-8 \times 3-4$. Stinkt.

Scleroderma cepa Vaill. - 30.8. - Wie S. verrucosum. Stachelige Sp. Feld.

S. verrucosum Bull. - 29.8. - Blassgelb, mit braunen Warzen bedeckt. Fruchtmasse wird viol.-schwarzbraun. Peridie lederig dünn. Dicke verzweigte Myzelstränge. Sp. grobwarzig, 12-15 im Diam. Feld.

Crucibulum vulgare Tul. - 17.8 .

Calvatia caelata Bull. - 22.6.

C. saccata Vahl. - 2.9. - Kopf deutlich abgesetzt.

Lycoperdon echinatum Pers. - 1.8 .

L. umbrinum Pers. - 14.8.

L. gemmatum Batsch -22.6 .

L. piriforme Schff. - 10.8 .

Bovista plumbea Pers. - 16.6.

B. nigrescens Pers. - 23.6.

Geaster coronatus Schff. - 27.8. - Innenkörper graubraun, oval, auf kurzem Sielchen knopfförmig abgesetzt, mit abgegrenztem Hof um die kegelige, faserige Mündung. Peridie nach dem Eintrocknen der fleischigen Schicht papierartig, normal in 4 Lappen gespalten. Sp. kugelig-warzig, 4-5, netzig, braun. Gemischter Sumpfwald.

G. pectinatus Pers. (striatus Cand. ss. Rick.) - 19.7.
Tremella foliacea Pers. - 27.8. - Harzbraun, blätterig-gekröseartig. Sp. weiss mit klcinem Stiel, $7-9 \times 5-7$. Auf Espe. (In Mustila 50 auf Nadelholz).

Dacryomyces stillatus Nees - 3.9. - Wellige, orangefarbene Scheibchen oder Schälchen mit einem Diam. von höchstens einigen $\mathrm{mm}$. Ohne oder mit nur angedeutetem F. Sp. ziemlich farblos, $16-20 \times 9-10$, kurze Wurstform. Nasser Wald, auf Holz. - Exs. vom 24.6.54. aus dem Gebiete Tuorila (Nachtrag c). Die daselbst gefundenen Ex. hatten $15-19 \times 8-10$ Sporen.

Calocera viscosa Pers. - 30.7 .

C. viscosa Pers. var furcata Fr. -21.7 .

C. palmata Schum. - 26.7. - H. $1-2 \mathrm{~cm}$ hoch orange, flachgedrückt, aufwärts verbreitet und in wenige kurze, flache Äste geteilt, gelatinösweich. F. häufig unten weisszottig. Sp. $8-9 \times$ 4-4.5. Auf Stämmen.

Clavaria botrytes Pers. - 15.8 .

C. aurea Schff. -29.7 .

C. flava Schff. -23.8 .

C. fennica Karst. - 9.8. - Fk. $10 \times 8$, reich verästelt. Strunk derb, violett mit weisser Basis. Zweige gelbbraun mit stumpfen Enden. Sp. $9-10.5 \times 5.25-5.75$. Bitterlich. Nadelwald.

C. condensata Fr. - 26.8. - Siehe Kap. 4. Ohne violette Töne.

C. condensata Fr. var. violaceo-tincta. - 29.7.

C. crispula Fr. - 28.8. - Fk. 1.5-3 hoch, reich verzweigt, ocker-lederfarben. Strunk minimal, weisszottig, wurzelnd. Endzweige gleichfarbig, gespreizt. Sp. $5 \times 3$, hellocker. Auf alten Stämmen, im Moose.

C. abietina Pers. - 19.8 .

C. Invalii Cott. et Wakef. - 7.8.

C. flaccida $\mathrm{Fr} .-22.8$.

C. gracilis Pers. - 2.9. - Fk. 4 hoch, blassweiss. Strunk sehr unbedeutend. Sp. 5-6×3-3.5, eintropfig. Anisgeruch. Im Moose.

C. rugosa Bull. - 22.8. - Fk. bis 10 hoch, weisslich, oft bloss einfach-keulenförmig, aber auch unregelmässig und schwach verästelt, abwärts verjüngt, aufwärts breitgedrückt. Auffallend runzelig. Stumpfe Spitzen. Sp. 7-9 6-8, bisweilen etwas grösser. Vielleicht eine Unterart von $C$. cristata. C. rugosa ist grösser, schwächer verästelt und runzelig.

C. cristata Holmsk. - 22.8. - Fk. 3-5 hoch, weisslich, bisweilen mit rötlichem Ton. Sp. $7-9 \times$ $6-8$, eintropfig, farblos.

C. lappa Karst. -25.8 . - Fk. 1-1.5 hoch. Sp. c. $8 \times 6$, eintropfig, einseitig meist etwas abgeplattet. An einem Grabenrande. Var. von C. cristata.

C. muscoides L. -3.9 .

C. fragilis Holmsk. (vermicularis Scop.) - 27.8. Reinweiss.

C. fusiformis Sow. - 28.8. - Fk. einfach-keulig, 3-6 hoch, kanariengelb, spindelförmig. Sp. rund mit Stielchen, 5 im Diam. Büschelig. Im Walde.

C. similis Boud. et Pat. (dissipabilis Britz.) - 23.8. Bis 4 lang, gelb, fast zungenförmig, $2 \mathrm{~mm}$ breit, zuletzt breitgedrückt und kanalisiert. Innen gelb. Sp. sternförmig-stachelig, 6-7 im Diam. Rasig, im Walde.

C. ligula Schff. -25.8 . 
Merulius tremellosus Schrad. - 27.7.

M. molluscus Fr. var. aurantiacus Klotzsch - 7.8. Farbe von Trametes cinnabarina Jacq. Sp. $7-8 \times 5.5$ $-6-7$, hyalin. Seitlich am Stamme einer toten Birke. Bestimmt von Prof $\mathrm{K} \mathrm{ujal}$ a.

Stereum hirsutum Willd. - 6.8.

S. rugosum Pers. - 4.9 .

Pleurodon auriscalpium L. -8.8 .

Dryodon cirrhatum Pers. -7.7

Hydnum repandum L. -6.7 .

H. rufescens Pers. - 19.7 .

Sarcodon imbricatum L. -12.8 .

S. subsquamosum Batsch, Quel., non Fr. - 1.9. H. bis 10 breit, schokoladenbraun, sammetig mit teilweise schuppig aufbrechender Oberfläche. Welliger Rand. F. $4 \times 20$, gleichfarbig. St. weisslich, bräunend mit weisslicher Spitze, $8 \mathrm{~mm}$ lang, tief herablaufend. Sp. $4.5-5 \times 4-4.5$, bräunlich, stachelig. Fl. schokoladenfarben, im Hute etwas blasser. Riecht. Bitterlich.

S. infundibulum Swartz - 29.7. - H. bis 10 breit, hell kastanienbraun, kahl, glatt, trichterförmig, mit aufgerichtetem Rand, fleischig-zäh. F. blass, abwärts dunkler und verjüngt, bis $10 \times 2$. St. weiss, bräunend, herablaufend, einige $\mathrm{mm}$ lang. Sp. $4-4.5 \times 3.5-4$, eckig-höckerig. Fl. weisslich. Starker, kräftiger Geruch. Nadelwald.

Calodon suaveolens Scop. - 18.7.

C. aurantiacum A. et S. -21.7 .

C. sulphureum Kalchbr. (geogenium Fr.) - 16.8. Zuerst schwefelgelb, dann bräunlich, höckerigzottig. F. rudimentär, gelb. St. und Fl. schwefelfarben. Sp. 3.5-4.5 $\times 3-4$, schwachkantig, eintropfig. Myzel schwefelfarben. Auf der Insel Vånà. Grabenrand.

C. ferrugineum Fr. -16.8 .

C. scrobiculatum Fr. -26.8 .

C. zonatum Batsch, ss. Bourd, et Galz. - 12.8, - H. bis 3 breit, undeutlich gezont, trichterförmig mit hellem, sterilem Rand, strahlig-runzelig. F. kurz und schmal, Farbe des $\mathrm{H}$., unten verdickt und dann verschmälernd und wurzelnd. Fl. bräunlich. St. werden rötlichbraun. Sp. sehr hellbraun, $4 \times 4,4.5 \times 4$ bis $5.5-4.5$. Zusammenfliessend. Nadelwald.

C. nigrum Fr. - 21.8. - Blauschwarz mit weissweisslichem Rand, filzig, ungezont. St. um $3 \mathrm{~mm}$ lang, weiss. Sp. $4 \times 4$, stachelig, farblos. Fl. schwarz.

C. cyathiforme Schff. -22.8 .

C. graveolens Fr. - 21.8. - H. bis 4 breit, weich, sehr dünn, sehr dunkelbraun mit weissem Rand, kaum sichtbar gezont, runzelig, trichterförmig. F. dunkel braun, $3 \times 3$, oben dicker. St. $1-1.5 \mathrm{~mm}$ lang, weiss. Sp. eckigrundlich, c. 4.5 im Diam., schwach punktiert, hyalin. Starker, würziger Geruch. Nadelwald. - Die Arten nigrum, melaleucum und qraveolens gehen nach $\mathrm{B}$ o $\mathrm{urd}$ o t et $\mathrm{Galzin}$ in einander über.

Phylacteria palmata Scop. - 24.8

P. terrestris Ehrh. -20.6 .

Polyporus ovinus Schff. -19.7 .

P. sulphureus Bull. - 16.6. - Dachziegelig wachsend, ohne F. oder ganz kurz gestielt. Hüte fächerförmig, bis 20 breit, ziegelrot-orange. F. grüngelb. Fl. gelblich, dann weiss. Sp. $6.5 \times 4.5$ etc. elliptisch oder kernförmig, weiss. Geruch von Gan. applanatum. Geschmack sauer. Auf lebenden oder toten Eichen.

Leucoporus brumalis Pers. - 16.6.

Spongipellis spumeus Sow. - 4.9. - H. bis 10 breit, halvkreisförmig, mehrere cm dick, gebuckelt, polsterförmig, striegelig-filzig, weiss, später gilbend, mit abwärts verlängerter Basis. R. weiss, bis 1 lang. Fl. schwammigweiss, wässerig. Sp. $7 \times 6$, mit einem Öltropfen und zugespitzter Basis. Der Pilz schwärzt überall. Auf lebender Linde.

S. borealis Vahl var. spathularia Karst. - 16.8. Bis 15 breit, weissgelb, beinahe polsterförmig. P. $5 \mathrm{~mm}$ lang. Sp. $4-5 \times 3$. Fl. $5 \mathrm{~mm}$ dick. Auf Nadelholz.

Leptoporus albidus Trog, Schff. (stipticus Quel.) 12.8. - H. bis 6 breit, 1 dick, weiss, runzeliggrubig, dreieckig -muschelförmig mit stumpfem Rand. Bisweilen fast gestielt. R. weiss, eng, abgerundet-eckig. Substanz weiss. Sp. elliptisch, gewöhnlich mit einer abgeflachten Seite, dazwischen wurstförmig, mit einem Tropfen, 3.5$4.5 \times 1.5-2.75$. Bitter. Auf Nadelholz.

L. adustus Willd. - 16.6 .

Phaeolus Schweinitzii Fr. - 23.7.

P. rutilans Pers. - 5.9 .

Coriolus pubescens Schum. - 11.7.

C. zonatus Nees. - 11.7.

C. versicolor L. -24.6 .

C. unicolor Bull. - 6.7.

C. abietinus Dicks. -22.7 .

Irpex sinuosus Fr. - 19.8 .

Lenzites quercina L. (Daedalea qu.) - 6.7.

L. betulina L. -21.7 .

L. sepiaria Wulf. -20.6 .

Trametes odorata Wulf. - 20.6.

T. squalens Karst. - (Pol. stipticus Pers.) - 21.7 H. 4-9 breit, mit stumpfem Rand, derb, zuerst weisslich, dann rotbraun, schliesslich schwarzbraun, uneben, bald kahl, polsterförmig. R. weiss. P. mittellang, gleichmässig eng, rundlich. Sp. $6 \times 4$. Fl. weiss, undeutlich gezont, zuletzt korkig, von auffallend zusammenziehendem Geschmack. Auf Nadelholzstümpfen, dachziegelig verwachsen.

Ungulina fomentaria L. - 17.6 .

U. marginata Fr. - 16.6

$U$. betulina Bull. - 26.6.

Ganoderma lucidum Leys. - 26.7.

G. applanatum Pers. - 17.6.

G. sp. - 31.7. - Mehrere lederzähe Hüte aus einer Wurzel, ohne F. Nierenförmig, kastanienbraun, sammetig-filzig, Mitte schwarz, Rand heller. Röhrenschicht hat nur Hautstärke (vielleicht weil die Ex. jung waren?). R. weisslich, bei Berührung braun. P. wie Nadelstiche, unter $0.1 \mathrm{~mm}$ breit (junge Pilze?). Sp. nur ganz wenige, oval, sehr dunkel (vermutlich braun), 5-6 $\times 3.5-4$. Auf altem Fichtenbalken. Von Prof. Kujala und mir als Ischnoderma benzoinum bestimmt. Irrtum, es sei denn, dass die Sp. nicht z. Pilze gehören und angeflogen waren.

Phellinus igniarius L. - 24.6.

Xanthochrous perennis L. -1.8 .

$X$. radiatus Sow. -19.8 . - H. 5-8 breit, sammetig, rostrot, mit feuerrotgelbem, abstehendem Rande, strahligrunzelig. R. olivbraun, $5 \mathrm{~mm}$ lang, eng. Keine Sp. Substanz wird rostbraun, lederholzig. Dachziegelig verwachsen. Auf toter Birke. 
Gyromitra esculenta Pers. - 16.6.

Cudonia confusa Bres. - 6.8.

Spathularia clavata Pers. - 3.9.

Rhizina inflata Schff. (undulata) - 5.7.

Otidea leporina Batsch - 25.8.

$O$. sp. (vielleicht $O$. onotica Pers.) - 5.9. - Bis zur weissen, filzigen Basis gespalten, mit schnekkenförmig eingerollten Rändern. Rehbraun. Beinahe gestielt. 4 hoch, ebenso breit. Sp. $10 \times 6$, eintropfig, farblos. Auf verfaultem Holz.

Sarcoscupha sp. (vielleicht identisch mit dem in Kap. 4 beshriebenen Pilz) - 2.9. - H. bis 2 breit, überall schwarzgrau. F. $5 \times 2-4$. H. (ausser Frucht schicht) und F. überall grauflockig bestreut. Sp. $22 \times 10$, farblos, glatt, ohne Öltropfen. Am 3.9. weitere Ex. mit beinahe schwarzem $6 \mathrm{~cm}$ langem F. Sp. bisweilen mit Öltropfen. Im Moose.

Lachnea hemisphaerica Wigg. - 30.8. - H. 1-1.5 im Diam., schüsselartig, aussen braunfilzig behaart. Innen hellgrau. Sp. $23 \times 13$, farblos, feinwarzig, mit einem grossen Öltropfen. Auf faulendem Stamm.

L. scutellata L. -7.7 .

\section{Gelegentliche Funde an anderen Stellen Finnlands}

Berücksichtigung fanden nur Pilze, die entweder in den Kap. 1-5 nicht erwähnt wurden, oder von mir für relativ selten angesehen werden.

\section{BASIDIOMYCETES.}

Hygrophoropsis aurantiaca Wulf. f. pallida Cke - Lohja. 13.9.50. - H. gelbocker. F. unten schwarzbraun, oben blass. L. crème. Wohl identisch mit flavens Karst.

Hygrocybe punicea Fr. - Paimio 45.

Lyophyllum connatum Schum. - Hiivola, bei Riihimäki. Herbst 51.

Clitocybe inornata Sow. - Lohja. 19.9.50.

C. splendens Pers. - Lohja, undatiert. - Falbgelb. Wird heute gewöhnlich mit C. gilva identifiziert.

C. catinus Fr. - Lohja. Undatiert. - Sehr unsichere Art. Wird heute bald mit $C$. cerussata, bald mit C. infundibuliformis in Verbindung gebracht.

C. angustissima Lasch - Hiivola. Herbst 51.

Omphalina chrysophylla Fr. - Lohja. 19.9.50 - Siehe Nachtrag c).

Tricholoma pessundatum Fr. - Paimio 46 und Hiivola, Herbst 51. - Siehe Nachtrg e).

T. terreum Schff. var. triste - Lohja. 17.9.53. Cortiniert.

Cantharellula obbata Fr. - Lohja, undatiert, und Hiivola, Herbst 51. - Kleiner als C. cyathiformis. Im trockenen Zustande mit bläulichem Schimmer. Fl. grau. Unsichere Art. Form von C. cyathiformis?

Lentinellus cochleatus Pers. - Lohja. 50. - Trichteroder löffelförmig. L. fleischrötlich, weit herablaufend. Anisgeruch.

Marasmius Wettsteinii Sacc. et Sydow - Hiivola, Herbst 51 .

Xeromphalina campanella Batsch var. badipes - Lohja. Undatiert. - Wurzel zwiebelartig. Bedeutungslose Form.
Lepista personata Fr. - Lohja. 49. - Ähnlich L. nuda, aber nur F. blauviolett.

Rhodophyllus limosus Fr. - Hiivola, Herbst 51.

R. minutus Karst. - Hiivola, Herbst 51.

Amanita vaginata Bull. var. crocea Quel. - Lohja. Undatiert. - Orange.

Macrolepiota gracilenta Krombh. (umbonata Schum.) Lohja 49. - Mit charakteristischem rotbraunem zitzenartigem Buckel. Sp. $12-16 \times 7.5-9.5$. Leider kein Exsikkat.

Leucoagaricus excoriatus Schff. - Hiivola, Herbst 51. Lepiota acutesquamosa Weinm. - Lohja, in einem Garten 50. - Spitzkegelige Schuppen. Stinkt.

Agaricus villaticus Brondeau ss. Bres., Lange, Pears. (urinascens Möll. et Schaeff.) - Hiivola 26.9.53. - H. bis 15 breit, weiss, gilbend, ziemlich glatt, aber schuppig aufbrechend. Rand oft gezähnt behangen. F. keulig, robust, ziemlich kurz. Ring hängend, einfach, dick. Sp. gewöhnlich $9 \times 6$. Fl. derb und bei Verletzung wenig veränderlich. Geruch unangenehm. Trotzdem essbar. Kultivierte Stellen.

Crepidotus calolepis Fr. - Naantali. 10.12.48. - H. lederfarben, mit dunkleren Schüppchen. Sp. $7 \times 5$. An Laubholzästen. Bestimmt von Dir. $\mathrm{N}$ y b e r g.

Cortinarius hircinus Bolt. ss. Rick. - Bromarv. Spätsommer 47. - H. 5-8 breit, violett. F. mit viol. Spitze, zwiebelknollig. L. lila. Sp. $8-9 \times 4.5-5$, warzig, punktiert. Fl, violett. Geophyllageruch. Phlegmacium purpurascens Fr. - Lohja 53. Nadelwald. 
Pholiota lubrica Pers. - Naantali, Dez, 48 und Hiivola, Oktober 51.

P. flavida Schff. - Hiivola 51 .

Psilocybe semilanceata Fr. - Lempäälä 44.

Psathyrella appendiculata Fr. - Lohja 50. - Ähnlich P. hydrophila. Holzgelb. Sp. $7-8 \times 4.5$. Auf Laubholz.

Coprinus radiatus Bolt. - Lohja. 50. - H. bis $7 \mathrm{~mm}$ breit, gelblich. Nur wenige L. Auf Mist.

C. phaeosporus Karst. - Lohja 51. - Nahe verwandt mit C. Friesii und $C$. domesticus. Weiss. Sp. $9-15 \times$ 5-9. Ganz unsichere Bestimmung.

Geaster pectinatus Pers. - Lohja. Undatiert. - Bestimmt von Dir. N y b e r g.

Calocera palmata Schum. - Hiivola. 28.9.51.

Clavaria fragilis Holmsk. - Paimio. 46.

Merulius lacrimans Wulf. - Hiivola. 12.11.50.

Calodon melaleucum Fr. - Lohja. Undatiert. - Weiss mit schwarzer Scheibe, dünn, schmächtiger als C. nigrum, ungezont. Sp. 3-4 im Diam., eckigrundlich, schwach puntiert. Viellecht nur eine Form von $C$. nigrum.

Polyporus leucomelas Pers. - Loppi. 7.10.51. - H. bis 10 breit, grau, schliesslich schwarz. F. grau, $4-8$ lang. R. weiss, grauend. Fl. weiss, schwach rötend, im F. schwärzend. Sp. $4 \times 4$, rundlich, höckerig. Nadelwald.

P. sulphureus Bull. - Ruissalo, 15.6. 53.

Melanopus elegans Bull. - Lohja. 6.6.52. - H. bis 10 breit, löwengelb, schwärzend, derb, gleichdick, bald holzig. F. an der Spitze blass, in der Mitte schmäler, kurz, excentrisch, nicht seitlich. R. blassgelblich, rundlich, $0.2-0.3 \mathrm{~mm}$ breit. Sp. $5-6 \times 2--3$, bisweilen mit Öltropfen, cylindrisch bis Sonnenblumensamenform. In einem Garten, auf totem Pflaumenstamm. Die Pilze hatten eine schuppigrunzelige, schildpattartige Oberfläche, die vermutlich lokal bedingt war.

Phaeolus Schweintzii Fr. - Bromarv. 47.

Irpex lacteus Fr. - Loppi. - 24.5.51. - Milchweiss, ergossen, lederig. Auf totem Birkenast. U'berwinternd. Bestimmt von Mag. $\mathrm{M}$ a $\mathrm{lm} \mathrm{s}$ t $\mathrm{r}$ ö m.

Phellinus salicinus Pers. (Fomess.) - Sippola - Heinola, Mai 49. - H. zum grösseren Teil aufgewachsen, nur der obere Teil $(1-2 \mathrm{~cm}$. breit) hütig abgebogen. Wird schwärzlich. Stumpfer Rand. Kurze R. Sp. nichı erhalten. Substanz rotbraun. Sehr hart.

Xanthochrous pini Brot. (Trametes p.) - Bromarv 47. - H. bis 10 breit, sehr dick, braun. Sp. $5.5 \times 4.5-5.5$. An Kieferstümpfen, meist dachziegelig.

X. abietis Karst. (Trametes a.) - Bromarv 47. Braun. R. weiss-bestäubt. Sp. $6 \times 4$, gelblich. Auf toter Fichte.

\section{ASCOMYCETES.}

Gyromitra gigas Krombh. - Lohja. 50. - Sp. 30-40 $\times 12-14$.

Mitrula phalloides Bull. (M. paludosa) - Bromarv. 47. - S. Nachtrag c).

Rhizina inflata Schff. - Bromarv. 47.

Discina ancilis Pers. - Lohja 49. - Bis 4 breit. Braunrussig. Bestimmt von Dir. Nyberg.
D. venosa Pers. - Hiivola. Mai 51. - Milchkaffeefarben, faltig-runzelig. Unterseite weissgelb, durch aufsteigende Rippchen faserig. Ohne F. Auf faulenden Stümpfen.

Aleuria aurantia Müll. - Hiivola 51, Herbst.

Sclerotinia tuberosa Hedw. - Lohja. 49 und 50.

\section{Nachtrag: Das Jahr 1954.}

\section{Zusätzliche Literatur:}

- Kühner et Romagnesi: Flore analytique des champignons superieurs, 1953.

- Overholts: The Polyporaceae of the United States, Alaska and Canada, 1953.

Es konnte auch Einblick genommen werden in

- S a yer: The North American cupfungi (Operculates), Supplement 1942.

- Sa yer: The North American cupfungi (Inoperculates) 1951.

- Velenovsky: Monographia Discomycetum Bohemiae 1934.
Es zeigte sich jedoch, dass die Ascomyzetenflora Böhmens von der unsrigen stark abweicht. Von den wertvollen Sayerschen Arbeiten stand der erste Teil (Operculates), der für uns besonders wichtig ist, nicht zur Verfügung. Er ist längst vergriffen.

Das Pilzjahr 1954 war - trotz Wärme und Feuchtigkeit im Sommer und Frühherbst nicht günstig. Offenbar ist das trockene Frühjahr der Entwicklung des Myzels, besonders der Boleti im Sinne der älteren Forscher, nicht förderlich gewesen. Mikroskopische Untersuchungen der Cyst. und der Haut und chemische mit Hülfe eines Dutzends Reagentien wurden 1954 beinahe täglich gemacht. Entsprechend ist das Niveau der Bestimmungen ein »moderneres» als in früheren Jahren. 


\section{a) Mustila.}

Ein Besuch in Mustila brachte folgende Neufunde:

$(2.4 .-6.5$.

Pseudohiatula conigena Pers. ex. Fr. - 6.5. - Vermutlich schon früher gefunden, aber als esculenta Wulf. registriert.

Galerina unicolor Fl. Dan. - 3.5. - Kleiner als G. marginata. $\mathrm{H}$. bis 2 breit, schwach gebuckelt und nur wenig gerieft. F. $3 \times 5$, von unten bräunend. L. breit. Sp. 7.5 5. A. verarb. Nadelholz.

Coprinus cineratus Quel.-20.4. - Sp. $10.5 \times 6.5$, mandelförmig. Wohl schon früher gefunden, aber als C. stercorarius (Sammelart) bestimmt. Mistbeet. Coriolus abietinus Dicks. -4.5 .

\section{b) Lohja}

Zwei wichtige Funde (16.5.):

Clitocybe rhizophora (Vel.) Joss. - Siehe Kap. 2.

C. vermicularis Fr. - Von C. rhizophora nur schwer zu trennen. Grösser und rötlicher. Sp. 4.5 $5.5 \times 3$.

\section{c) Gebiet Tuorila bei Karkkila.}

21.6. bis 30.7 und 13.8. bis 2.9. Schöne, aber etwas eintönige Wälder überwiegend Fichte und Birke, letztere häurig von hoher (Furnier-) Qualität.

\section{BASIDIOMYCETES.}

Suillus luteus L. - 14.7.

S. granulatus L. -21.7 .

S. bovinus L. -20.8 .

S. variegatus Sow. -14.8

S. piperatus Bull. -8.7 .

Leccinum aurantiacum Bull. - 18.7.

L. rufescens Secr. - 14.7.

L. leucophaeum Gilb. - 19.7 .

L. leucophaeum Gilb. var. coloripes Singer (blau) 23.7 .

Boletus (besser vielleicht Xerocomus) versicolor Rostk. (sanguineus With. ss. Kallenb.) - 28.7. - H. bis 6 breit, blutrot, sammetig-filzig. F. bis $7 \times 8$, oben gelb, unten auf blassem Grunde rotfilzig mit gelblicher, wurzeinder Basis. R. gelb, durch Druck blaugrün, weit, eckig. Sp. $12 \times 4.5-5$ etc. Fl. blassgelb, blauend. Unter Birken.

B. edulis Bull. - 26.7.

Tylopilus felleus Bull. - 29.7.

Xerocomus badius Fr. -17.8 .

$X$. chrysenteron Bull. -22.8 .

X. subtomentosus L. -20.7 .

Paxillus involutus Batsch - 3.7

P. filamentosus Fr. (leptopus Fr.) - 2.7. - Wurde von mir erstmalig 46 in L,ohja unter Ellern gefunden und Dir. Nyberg zugesandt. Wir sahen in dem Pilz damals eine Form des $P$. involutus, dem er sehr nahe steht. Nordischer Pilz. H. glatt, braungelb mit dunkleren Schuppen, bis 8 breit. F. kurz und nach unten verschmälernd. L. gelblich, kaum fleckend. Sp.6.5 4.5. Fl. gelb. Unter Ellern.

$P$. alrotomentosus Batsch -24.8

Hygrophoropsis aurantiaca Wulf. - 20.7.

Gomphidius glutinosus Schff. - 14.8.

Hygrophorus piceae Kühn. - 19.8 .

H. olivaceoalbus Fr. - 17.8 .

Camarophyllus pratensis Pers. - 31.8 .

Hygrocybe nitrata Pers. -21.8 .

H. nigrescens Quel. -14.8 .

H. conica Scop. - 14.8.

H. Langei Kühn. (obrussea Rick., constans Lange, amoena R. Sch.) - 29.7. - Wie H. conica, aber nicht schwärzend. Sp. $10-12 \times 6-7$.
H. chlorophana Fr. -31.8 .

H. psittacina Schff. -31.8 .

H. coccinea Schff. -31.8

H. cantharella Schw. -29.7 .

H. miniata Fr. -21.7 .

Clitocybe Alexandri Gill. - 27.8.

C. odora Bull. - 17.8 .

C. clavipes Pers. -15.8 .

C. squamulosa Pers. - 7.7.

C. infundibuliformis Schff. -17.7 .

C. phyllophila Fr. - 18.8 .

C. pithyophila Secr. - 19.8 .

C. rivulosa Pers. -28.7 .

C. tuba Fr. -20.8 .

C. candicans Pers. f. phyllophila -.. 16.8 .

C. fragrans Sow. -25.8 .

C. hydrogramma Fr. - 18.8 .

Laccaria laccata Scop. -8.7 .

L. proxima Boud. -29.8 .

Tricholomopsis platyphylla Pers. -2.7 .

T. rutilans Schff. - 12.7.

T. decora Fr. -2.9 .

Collybia cirrhata Schum. var. typica R. Maire - 17.8.

G. hariolorum Fr. - 15.7. - Wie C. confluens, aber starker Zwiebelgeruch

C. confluens Pers. -3.7

C. macilenta $\mathrm{Fr} .-18.8$.

C. ocellata Fr. -2.9

C. acervata Fr. - 11.7. - H. bis 6 breit. Sehr hygrophan. Fleischrot, trocken beinahe weisslich. F. dunkelrot, $12 \times 2-4$. L. rötlich, dann bleich. Sp. 5-6 $\times 2.75-3$. Schwach bitterlich. Auf totem Nadelholz, büschelig, durch striegelige Basis verbunden.

C. dryophila Bull. - 1.7

C. funicularis Fr. -4.7 .

C. aquosa Bull. - 25.6 .

C. asema Fr. -24.8 .

C. butyracea Bull. - 16.8 .

Omphalina chrysophylla Fr. - 26.8. - H. bis 6 breit, braungelb, trocken, gelbgraulich, genabelt, flokkig, schuppig. F. orangegelb, oft breitgedrückt. L. orangefalb. Sp. $9-11 \times 5.5-7$, gelb. Auf Nadelholzstamm

O. umbellifera L. -4.7 .

O. umbellifera L. f. albida -22.8 . 
O. grisella Weinm. - 7.7. - H. bis 1 breit, gelbbraun, gerieft-gekerbt. F. $3 \times 1$, gleichfarbig mit Basis weisslich. L. weisslich. Sp. $7-8 \times 4-4.5$. Im Grase.

O. sphagnicola Berk. - 1.7. - H. 1 breit, braun. Mitte etwas schuppig, trichterförmig, durchscheinend gerieft. F. etwa Farbe des H., vielleicht etwas heller. I. graulich, weit herablaufend. Sp. beinahe boletenartig, $8-9.5 \times 3.5-4$. Auf Sphagnum.

O. scyphiformis Fr. ss. Langei - 22.8 .

Armillariella mellea Vahl. - 14.8.

Tricholoma flavobrunneum Fr. - 15.8.

$T$. viridilutescens Moser - 19.8. - H. bis 6 breit, grün bis gelbgrün, mit oliv- bis schwarzen radialen Fasern. F. weisslich, dann gelb, bis $8 \times 12$. L. weiss, am Hutrand gelb, gesägt. Sp. c. $6 \times 5$. Mild. Geruchlos. Nadelwald. T. fucatum und sejunctum sehr nahe stehend.

T. inamoenum Fr. -14.8 .

T. album Schff. -25.8 .

T. saponaceum Fr. var. ardosiacum Bres. - 23.8.

T. virgatum $\mathrm{Fr} .-20.8$.

Cantharellula umbonata Gmel. - 18.8 .

Melanoleuca strictipes Karst. - 6.7.

M. melaleuca Pers. - 21.7.

Lentinellus bisus Quel. - 28.7. - H. bis 4 breit, grauledergelb, ausblassend, mit tiefem, braunem Nabel, gerieftem und gelapptem Rand. F. rostfarben, längsfurchig. L. weisslichgrau, herablaufend. Sp. 5-5.5×4, raub. Zwischen Moosen an Holzstücken.

Panus conchatus Fr. - 5.7.

Mucidula radicata Relh. -4.9 .

Mycenella bryophila Voglino - 18.8. - H. 1 breit, braungrau und gerieft, glockig. Rand heller. F. $2 \times 0.5$, fein bereift, bräunlich. L. weisslich. Sp. $6 \times 5$ etc. warzig. Gespaltene Cyst. konnten nicht festgestellt werden. Auch liess es sich nicht feststellen, ob der Pilz wurzelte oder nicht. Dic nicht wurzelnde Form $=M$. trachyspora Rea.

M. lasiosperma Bres. ss. Kühner. - 1.9. - H. bis 1 breit, glockig, braun. F. mit Wurzel, $6 \times 0.5$, oben weiss, unten bräunend. L. weisslich. Sp. warzig, sehr unregelmässig, c. $5 \times 4$ etc. Cyst. schopfig, in einigen Fällen konnte eine schwer wahrzunehmende Spaltung der Spitzen festgescellt werden.

Michromphale perforans Hoffm. - 22.6.

Pseudohiatula conigena Pers. ex. Fr. (stephanocystis Kühn. \& Romagn.) - 26.6. - Sp. $7 \times 4$, nicht verbogen. Cyst. stumpf. Mild. Auf Kieferzapfen.

P. tenacella (Pers.) Fr.ss. Schroet., Favre. - 6.7. -Sp. $6 \times 3$, einseitig abgeplattet. Cyst. spitz. Auf Kieferzapfen. Bitterlich.

Marasmius scorodonius Fr. - 15.7 .

M. oreades Bolt. -30.7 .

M. Wettsteinii Sacc. et Sydow -- 4.7.

M. androsaceus L. -1.7 .

Xeromphalina campanella Batsch - 19.7.

Mycena haematopoda Pers. - 21.8.

M. galericulata Scop. -23.6 .

M. rugosa Fr. - 1.7.

M. epipterygia Scop. - 19.8 .

M. citrinomarginata Gill. (lineata Fr. ss. Rick.) - 16.7 .

M. rubromarginata Fr. - 15.7 .

$M$. rosella Fr. - 14.7

M. purpureofusca Peck (janthina Fr. ss. Rick.) - 17.7. H, 1-2 breit, braunviolett. Mitte rotbraun.
Glockig-kegelig, runzelig-gefurcht, bisweilen gebuckelc. F. gleichfarbig, bis über $10 \times 2$. L. mit lilaviol. Schneide. Sp. ecwa wie bei $M$. rubromarginata. Cyst. schmal. Auf Strünken und im Moose. Steht $M$. rubromarginata verdächtig nahe.

M. janthina Fr. non Rick. - 29.8. - H. bis 1.5 breit, glockig-kegelig, bräunlichviolett. Mitte eher braun. Gerieft. F. einige $\mathrm{cm}$ lang und um $1 \mathrm{~mm}$ breit, dunkelviolett. L. blass mit violettlichem Ton. Sp. c. $11 \times 7$. Im Moose.

M. pura Pers. - 1.7.

M. laevigata Lasch -27.7 .

M. alcalina $\mathrm{Fr}$. var. chlorinella Lange (leptocephala ss. Rick.) - 23.6 .

M. olivascens Quel. ss. Oort (lineata Bull. ss. Lange et Smith) - 23.7. $-\mathrm{H}$. bis 1 breit, oliv. Rand heller. Hygrophan. Wird gelblich. Gerieft. F. $2-3 \times$ unter 1, bräunlich, faserig gestreift, aber kahl. L. strohfarben, bauchig angewachsen, ziemlich frei. Sp. $8-8.5 \times 4.5-5$. Gyst. eiförmig. Auf Holzstücken.

M. aetites Fr. ss. Rick. - 15.7 .

M. alcalina Fr. - 4.7.

M. flavoalba Fr. Weisse Form. - 20.7.

M. fibula Bull. - 11.7 .

M. gypsea Fr. -20.7 .

Clitopilopsis fallax Quel. - 23.8. - H. 4 breit, konvex, dann niedergedrückt. Rand eingebogen. Schmutzigweiss, filzig. F. bis $4 \times 10$. Basis weissfilzig. L. weisslich. Sp. $5-5.5 \times 3-3.5$. Bitter. Geruch des Clilopilus prunulus. Sieht wie dieser aus, nur kleiner und fester.

Cilitopilus prunulus Scop. - 25.7.

Rhodophyllus sheculus Fr. ss. Kühn. et Romagn. 17.8. - H. bis 3 breit, blass graubraun, trocken weiss. Rand gerieft. F. bis $6 \times 5$, weiss, gerieft, gebrechlich. L. zuerst weisslich, dann rötlich. Sp. beinahe rund, $8 \times 8$ etc.

R. repandus Bull. - 29.7. - Nicht hygrophan. H. 5 breit, hellederfarben, schwach gebuckelt. F. weiss, $6 \times 8$. L. rosa. Sp. isodiametrisch, bis $8 \mathrm{im}$ Diam. Mehlgeruch. Im Freien. Feldrand. Nach Kühn. et Romagn. = prunuloides $\mathrm{Fr}$.

R. clypeatus L. -19.8 .

$R$. aprilis (Britz.) Romagn. (majalis Lange) 28.7. - H. bis 4 breit, stark gebuckelt. Rand gerieft. Schmutzigdattelbraun, trocken schmutziggrau. F. $3-7 \times 3-10$, gerieft, weisslichblass, bisweilen verdreht. L. fast frei. Sp. beinahe rund, $8 \times 7$ etc, Gesellig im Nadelwalde.

R. nidorosus Fr. -15.8 .

R. rhodopolius Fr. - 15.8. - Mit langem, weissem, silbrig glänzendem $\mathrm{F}$.

$R$. staurosporus Lange -17.8 .

R. mammosus Fr. Quel. ss. Konr. et Maubl, - 17.8.

$R$. mammosus Fr. var. sericoides Kühn. - 22.8. Sehr fest. H. 3 breit, dunkelbraun, gerieft, konkav mit Papille. F. $5 \times 6$, sehr dunkel, unten verdickt. L. breit, bauchig, ausgebuchtet angewachsen. Sp. bisweilen beinahe sternförmig, bis $10 \times 8$. Cyst. an der Schneide.

R. papillatus Bres. - 21.8. - H. etwa 2 breit, braun, riefelig, glockig, mit warzigem Scheitel. F. bis $6 \times 1$, beinahe schwarz. Basis weisswollig. L. schmutzigrosa, ziemlich breit, nicht gedrängt. Sp. oval z.B. $8 \times 5.5,10 \times 6.5$ etc., sehr unregelmässig. Steht zwischen $R$. papillatus ss. Langei und clandestinus ss. Langei. 
R. junceus Fr. -26.7 .

R. icterinus Fr. -5.7 . - H. 2 breit, hellhoniggelb resp. hellolivgelb, radial seidenfaserig, kaum oder nur schwach hygrophan. Wird weisslicher. Kleine Papille. F. $8 \times 3$, faseriggestreift, kahl, Farbe des H., zerbrechlich. L. werden rötlich, aufsteigend, bauchig, ausgebuchtet. Sp. $8-10 \times$ $6-7$, meist sechseckig. Im Walde.

$R$, infulus Fr. - 29.7.

$R$. cetratus Fr. ss. Fr. non Rick, $-30.7 .-$ H. 4 breit, ockerfuchsig resp. zimtgelb, gerieft, mit flachem Buckel. Mitte dunkler. F. $9 \times 7$, strohfarben (bräunlich), zäh, unten weisslich, seidig gestreift. L. blass, mit rötlichem Ton, ausgebuchtet angewachsen. Sp. $8-11 \times 6-8$.

R. placidus Fr. var. gracilis Lange -24.7 . - H. bis 4 breit, glockig, genabelt, dunkel schwarzbraun, nicht gerieft, aber sparrig-geschuppt, besonders in der Mitte, die beinahe schwarz sein kann. Nicht hygrophan. F. $5 \times$ einige mm, nach oben verdickt, schwarzblau, faserig gestreift, kahl, unten weissfilzig. L. breit ausgebuchtet, rötlich. Sp. $10-11 \times 5.75-6.5$. Keine Cyst. auf der Scheide. Geruch süsslich (schlechtes Mehl?). Auf oder neben Holzstücken.

R. asperellus Fr. -23.7 .

R. serrulatus Fr. - 14.8. - H. bis 2.5 breit, schwarzblau, genabelt. F. blau bis schwarz. L. mit schwarzer, gesägter Schneide. Sp. $9 \times 7$ etc.

R. lampropus Fr. -22.8 .

R. chloropolius Fr. ss. Romagn. - 1.9. - H. 1.5 breit, hellbraun mit Olivton, mit dunkelbrauner, niedergedrückter, beinahe genabelter Mitte, gerieft. F. $3 \times 1$, kahl, hellbraun mit Olivton. L. schwach bräunlich gerandet. Sp. $11 \times 7$ etc., sehr unregelmässig. Viehweide.

R. minutus Karst. - 16.8 .

$R$. carneoalbus With. - 19.8. - H. bis 2.5 breit, gewölbt, genabelt, weisslich mit gelblichem Ton. F. bis $5 \times 2$, ganz weiss. L. zuerst weiss, dann rötlich, schwach herablaufend. Sp. $9-11 \times 7-8$ etc.

$R$. sericellus Fr. -17.7 . - H. 1 bis 2 breit, elfenbeinweiss, seidig. F. blass, im Alter dunkelrötend, $4 \times 2$. L. weissrosa, bald angewachsen, bald herablaufend. Sp. $8 \times 8,9 \times 7.5$ etc. Grasige Stellen.

Pluteus cervinus Schff. - 12.7.

$R$. atromarginatus Konr. - 24.7.

R. minutissimus Maire, f. major Kühn. - 3.8. - Der Gruppe Hispidnderni angehörig. H. 4 breit, gelbbraun mit rötlichem Ton, glockenförmig, faserig. F. überall gepudert, $10 \times 3$, sehr hart und zäh, oben rötlich, in der Mitte durch lose Fasern dunkelbraun, unten beinahe ocker und bis $6 \mathrm{~mm}$ verdickt. $6 \mathrm{~cm}$ lange und $1 \mathrm{~mm}$ breite ockergelbe Wurzel. L. schmutzigoliv mir rötlichem Ton. Sp. 5-5.5 $\times 4-4.5$, unter dem Mikr. hyalin, en masse rötlich. Cyst, auf der Schneide formlos, gross und aufgeblasen, auf der Fläche der L. flaschenförmig (oben 6 breit). Jodoform- oder Rettichgeruch. Mischwald.

Volvariella speciosa Fr. - 13.7.

Amanita vaginata Bull. var. badia Schaeff. - 18.7.

A. vaginata Bull. var. fulva Schaeff. ex. Pers. - 18.7.

A. vaginata Bull. var alba $\mathrm{Fr},-30.7$.

A. crocea Quel. - 19.7.

A. muscaria L. -14.8 .

A. regalis $\mathrm{Fr} .-26.7$.

A. virosa $\mathrm{Fr} .-20.8$.
A. porphyrea A. et S. -25.7 .

A. rubescens Pers. -21.7 .

Macrolepiota procera Scop. - 18.8 .

M. rhacodes Vitt. - 17.8.

Lepiota alba Bres. - 18.8.

L. clvpeolaria Bull. - 13.8 .

L. clypeolaria Bull. var ochraceosulphurescens Locq. 18.8. - Ockergelbe Töne. Sp. bis $16 \times 6$.

Agaricus campestris L. var. squamulosus Rea. - 24.7. Braune Schüppchen.

A. arvensis Schff. -30.8 .

A. silvicolus Vitt. -27.8 .

A. rubellus Gill. - 19.8.

A. comtulus Fr. - 1.9. - H. 3 breit, gelblich weiss F. $2 \times 6$, weiss. L. rosa. Fl. kaum gilbend. Sp. $4-5.5 \times 3-3.5$. Anisgeruch. Viehweide.

Cystoderma carcharias Pers. - 25.8.

C. amianthinum Scop. var. typicum - 16.8. - Kleinsporig.

C. cinnabarinum A. et S. -18.8 .

Ripartites tricholoma A. et S. - 18.8 .

R. helomorphus Fr. - 16.8 .

Tubaria pallidispora Lange ss. Kühn. et Romagn. 6.7. - H. 1 breit, gerieft, wenig mehlig, fleichfarben. F. gleichfarbig, $3 \times 1$. Sp. sehr blass, breit mandelförmig, $7 \times 5$. Auf Holz. NB. Seit Romagn. für $T$. pallidispora Lange und conspersa Fr. mandelförmige und für $T$. pellucida und furfuracea ss. Romagn. elliptische $\mathrm{Sp}$. festgestellt hat, ist eine sichere Scheidung dieser Gruppen möglich geworden. Diese Möglichkeit lag bei meinen früheren Bestimmungen nicht vor. $T$. pallidispora und conspersa sind jedoch nach wie vor häufig nicht zu trennen. So auch im vorliegenden Falle.

T. minuıalis Romagn. (minima Lange) - 18.8. Úberall zimtocker. $H$. einige $\mathrm{mm}$ breit. $\mathrm{F}$. $1-2 \times 0.5-1$. Basis weisslich. Sp. $5.5-6 \times 3-4.5$. Kurze Gyst., meist keulenförmig, $30 \times 8$ etc. Auf Holz.

T. pellucida Bull. ss. Romagn. - 4.7. - H. um 1 breit, fleischrötlich, kahl, hygrophan. Rand schwach gerieft. F. gleichfarbig, $3 \times 1-2$. Basis weiss. L. ziemlich gedrängt und herablaufend. Sp. 5.5-6.5 $\times 4-4.75$, elliptisch. Auf Holz.

T. conspersa Pers. - 4.7. - H. bis 2 breit, zimtrötlich, mit weissen Velumresten bedeckt, kaum hygrophan. Rand bisweilen undeutlich gefurcht. F. zuerst mit weissen Flöckchen bedeckt, Basis weissflockig. L. mit weisslicher Schneide (keulenförmige Cyst.). Sp. mandelförmig, c. $7 \times 5.5$.

Rozites caperata Pers. - 29.7.

Hebeioma fastibile Fr. - 20.8 .

H. testaceum Batsch -2.9 .

H. crustuliniforme Bull. -25.8 .

H. pusillum Lange - 29.8. - H. 2.5 breit, spitz oder stumpf gebuckelt, rötlichbraun. Buckel dunkler. F. $3-6 \times 2-4$, obere hälfte weiss gepudert, unten bräunlich, ziemlich hart, schwach faserig gestreift. L. tonbraun, tränend, gekerbt. Sp. $12 \times 7$, elliptisch bis citronförmig, rauh. Rettichgeruch. Bitterlich. Nadelwald.

Inocybe obscura Pers. - 2.7.

I. cincinnata $\mathrm{Fr} . \quad-11.7$

I. pusio Karst. -27.6 .

I. geophylla Sow. -18.8 . 
I. fastigiata Schff. -25.8 .

I. Cookei Bres. - 18.7 .

I. descissa Fr. var. brunneoatra Lange - 16.8.

I. hirtella Bres. - 26.8. - H. 1 breit, gelblich, zuers konisch, dann ausgebreitet, mit oder ohne Buckel, faserstrelfig. F, gleichfarbig, $3 \times 1$. Äusserste Basis verdickt, sub lente faserstreifig. L. werden bräunlich. Sp. $8.5 \times 5$ etc. Lange flaschenförmige Cyst. $(70 \times 15)$.

I. posterula Britz. ss. Sacc. -22.8 .

I. lacera Fr. -4.7 .

I. asterospora Quel. - 6.7.

I. xanthomelas Kühn. -- 18.7 .

I. praetervisa Quel. - 21.7.

I. napipes Lange - 13.7.

I. umboninota Peck. -21.7 .

I. umbrina Bres. - 11.7.

I. descipientoides Peck. ss. Kühn. et Romagn. (globocystis Vel.) - 18.7. - H. 4 breit, gebuckelt, hraun, radialfaserig. Mitte glatt. F. $4 \times 7$, faserig gestreift. L. bräunlich. Sp. meist sehr schwach höckerig, $9 \times 8,8 \times 7$ etc. Cyst. teilweise sehr dick. Steht I. Boltoni sehr nahe.

I. pusilla Bres. - 18.7.

I. lanuginosa Bull. f. typica Hein - 20.7.

I. rufoalba Pat. et Doass. (Jacobi Kühn.) - 18.7. H. 2 breit, gebuckelt, braun, faserig gestreift. F. weisslich, $3 \times 5$. Sp. schwach höckerig, bis $7-8 \times$ 5. Cyst. spulen- resp, flaschenförmig, sehr schmal.

I. proximella Karst. - 15.7. - H. bis 4 breit, blassbraun mit hohem dunklem Buckel. F. voll, $6-8 \times 3-4$, häufig mehrfach verbogen, faserig, ohne Knolle. Sp. $6-8 \times 5-7$, eckig-zapfig, sehr unregelmässig. Cyst. $57-65 \times 18-21$, bauchigspulenförmig. Geophyllageruch. Strittige Art, neuerdings von Konr. et Maubl. wieder als selbständige Art anerkannt.

Alnicola submelinoides Kühn. - 27.8. - H. 1 breit, braun, gerieft, hygrophan. F. rötlichbraun. Spitze heller, $3 \times 1$. L. zimtbraun. Sp. c. $10 \times 5.5$, mandelförmig, deutlich punktiert. Cyst. stumpfkeulenförmig. Unter Ellern.

Galerina tibiicystis (Atk.) Kühn. - 26.7. - H. 1-2 breit. F. $10 \times 1$ etc. Sp. warzig, $8.5 \times 5.5$ etc. Cyst. mit kleinen runden Köpfchen $(3-7-11$ breit). Kein Mehlgeruch. Ich wende hier zum ersten Mal die Kühnersche Systematik der im Sumpfe wachsenden Galerinas an. Welcher Art (nach Kühner) die unten erwähnte G. hypnorum angehört, lässt sich nachträglich nicht mehr feststellen.

G. paludosa Fr. - 29.6. - H. bis 1.5 breit, bis zur Hälfte durchscheinend gerieft, olivhonigfarben. F. etwa gleichfarbig (braun), sehr lang, mit vergänglichen weissen Velumresten. L. rostfarben, angewachsen. Sp. etwa $8-10 \times 6-7$, ei- bis subcitronförmig. Auf Sphagnum, im Sumpf. Früh.

G. hypnorum Schrank - 20.7.

G. mevcenopsis Fr. - 15.8 .

G. triscopa Fr. ss. Karst. - 13.7. - H. bis 1.5 breit, braun, mit Buckel. Hygrophan. Trocken ocker. Rand gerieft. F. verbogen, $3 \times 1$, rostfarben, unten umbra. Bisweilen weissstriegelig mit Substrat verbunden. L. dünn, dicht, rostfarben. Sp. hellrostfarben, $6 \times 4$ etc. Auf Holzstücken.

G. marginata Batsch -16.8 .

Phaeocollybia Christinae Fr. - 17.8. - H. bis 3 breit, feurig zimtorange. Rand dunkler. F. safran- bis braunblutrot, oben gewöhnlich heller, $8 \times 3$. L. dicht, bauchig, schliesslich safrangelb. Sp. $4-5 \times$. $2.5-4$.

P. Jennyae Karst. - 14.7. - H. bis 3 breit, braun, glatt, gerieft. Höchst hygrophan. Trocken glänzend ledergelb bis ocker, ungerieft. F. bisweilen wellig, schwarzrot, $6 \times 2-3$, gerieft, unten weissfilzig, wurzelnd. L. braun, beinahe gerade (horizontal) angewachsen, sehr dicht, c. $1 \mathrm{~mm}$ breit. Sp. $5.25-6 \times 3.75-4.25$, rostgelb, eiförmig. Im Moose des Nadelwaldes.

Gymnopilus sp. - 20.7. - H. bis 5 breit, rostbraun, ziemlich glatt mit vergänglichen Velumresten. F. weisslichblass, faserig gestreift, unten weissfilzig, bis $3 \times 8$. L. goldgelb, angewachsen, dann ausgebuchtet. Sp. vvarzig, $8 \times 5$. Gyst. haarförmig $27 \times 3$, Apex köpfig, 6-7 breit, sehr ähnlich denjenigen des $G$. hybridus. Fl. gelb. Geruch nicht auffallend. Bitter. Büschelig auf einem alten Birkenstamm. Vermutlich $=G$. spectabilis Fr. var. junonia Fr.

G. liquiritice Pers. -- 21.7.

G. penetrans Fr. - 18.7.

Dermocybe concinna Karst. - 30.8. - Überall gelbliche Töne. H. bis 5 breit, rostrot, gebuckelt, wogig-runzelig, am Rande niedergebeugt, filzig. F. bis $9 \times 9$, gleichdick, kahl, rostrotfaserig. L. werden rostrot, breit, ausgebuchtet ange wachsen. Sp. $9-11 \times 5.5-7$, warzig, mandelförmig. dickwandig. Fl. gelb. Rettichgeruch.

D. cinnamomea L. var. conformis Fr. f. croceifolia Peck. -14.8 .

D. cinnamomea L. var. lutescens Hry - 19.8.

D. cinnamomea L. var. paludosa Fr. - 30.7.

D. semisanguinen Fr. -29.7 .

D. miltina Fr. ss. Quel., Rick. - 24.8.

D. sanguinea Wulf. - 14.8 .

Cortinarius violaceus L. -18.8 .

C. traganus Fr. -8.8 .

C. alboviolaceus Pers. -28.7 .

C. hircinus Bolt. ss. OvS. -29.8 . - H. 5 breit, gelbbraun, mit blassem eine Spur bläulichem, seidigem Rand. F. $6 \times 10$. Knolle 15 breit. Faserig gestreift, oben schwach violettlich. L. zuerst bläulich, dann rostfarben. Sp. $9.5-11 \times 5-5.5$, mandelförmig. Zahlreiche Cyst. auf der Schneide. Reaktion auf $\mathrm{KOH}$ und Ammoniak null, auf Phenol rot. Fl. nicht violett, sondern blass. Geruch etwa von G. camphoratus.

C. camphoratus Fr. - 14.8.

C. pholideus Fr. -20.8 .

C. spilomeus Fr. -24.8 .

C. spilomeus Fr. var. depauperatus Lange -- 29.8 .

C. anomalus Fr. -25.8 .

C. raphanoides Pers. -13.8 .

C. gentilis Fr. -17.8 .

C. callisteus Fr. - 18.8

Hydrocybe armillata Fr. - 17.8.

H. paragaudis Fr. -23.8 . $-\mathrm{H}$. bis 7 breit, fuchsig bis rotbraun, feinschuppig. F. bis $8 \times 10$, mit roten, unvollständigen, undeutlichen Gürteln auf bräunlichem Grund. L. rot-schokoladenbraun. Sp. $7-8 \times 5-6$, aber auch beinahe rund, z.B. $6 \times 5.5$, warzig.

$H$. saniosa Fr. ss. Langei et Moseri - 30.7.

H. saniosa Fr. var. paludosa R. Sch. (saniosa Rick.?) 14.8. - H. bis 5 breit, zuerst spitz-, dann flachgebuckelt, fachsig, kaum hygrophan, seidenfädig. F. bis $12 \times 8$, faserig, rostfarben-gelblich. 
Auch innen gelblich. Sp. sehr wechselnd, gewöhnlich mandel- bis eiförmig, $9-10 \times 6-7$.

H. limonia Fr. - 19.8. - Dieser hygrophane, nördliche Pilz wird von vielen Autoren, die ihn nie gesehen haben, mit $C$. callisteus zusammengeworfen. $\mathrm{Zu}$ Unrecht. $\mathrm{H}$. bis 4 breit, braunrot, zu feuerrotgelb ausblassend, mit gelben Velumresten. Huthaut mit Lauge schwarz. F. bis $8 \times$ $6-12$ (unten), auf gelbem Grund rot überfasert. Gelbe Ringzone. L. zeigen anfangs ähnliche gelbrötliche Töne. Sp. etwa $9 \times 7$, schwach warzig.

H. evernia $\mathrm{Fr},-13.7$. - H. bis 6 breit, kastanienbraun/violettlich, nicht sehr stark ausblassend. Rand bei jungen Ex. weiss-seidig, durch Cortina. F. $10-12 \times 10$, meist gleichdick, unten bisweilen verschmälernd, walzenförmig, ganz violett, aber ausbleichend, mit vergänglichen weisslichen Schuppen, aber häufig auch einfach oder mehrfach gegürtelt. L. sehr breit (bis $15 \mathrm{~mm}$ ). Sp. mandelförmig, $9-10 \times 5.5-6$, punktiert. Schwacher Rettichgeruch.

H. scutulata Fr. -26.7 . - H. 3 breit. F. $10 \times 8-10$ (unten). L. breit und entfernt. U'berall, auch Fl. violett. Sp. 8.5-9.5 ×5.25-5.5, mandelförmig, punktiert. Geruch süsslich/rettich. Vielleicht nur eine kleine Form von $H$. evernia.

H. bicolor Cke -27.8 .

H. parevernia Hry. - 24.8. - H. bis 6 breit, ziemlich hellbraun. Trocken lederfarben-ocker. F. unten beinahe schmäler als oben, bis $8 \times 8$, weisslich, nur angedeutet violett. Undeutliche Ringzone. I. Farbe der H. Sp. 9-12×5-6, mandelförmig. Rettichgeruch. Reaktion auf Phenol purpurn.

H. torva Fr. -29.8 .

H. saturnina Fr. ss. Kühn. et Romagn. -- 29.7. $\mathrm{H}$. bis 7 breit, kastanienbraun, nur wenig entfärbend. F. mehr oder weniger cylindrisch, bis $10 \times 10$. Ganz unten leicht spindelig, oben violett, unterhalb weisslich und ganz unten weissfilzig. L. sehr breit, anfangs violettlich, schliesslich kaffeebraun mit schwach-violettlichem Ton, gesägt (sterile Zellen). Sp. 10-11.5 $\times 5-5.5$, mandelförmig, warzig.

H. castanea Bull. - 23.8 .

H. cypriaca Fr. ss. Rick. - 24.8. - H. bis 6 breit, blass rötlichbraun, zuerst gebuckelt, dann flach, bräunlich behangen. F. bis $10 \times 8$, weissviolett. L. anfangs violettlich. Sp. mandelförmig oder elliptisch, c. $10.5 \times 6$. Wird von einigen Autoren mit $H$. bicolor identificiert.

H. sciophylla Fr. ss. Hry - 30.8. - Nicht sehr hygrophan. H. 3 breit, dunkelbraun mit viol. Ton. F. zuerst violett, später bräunlich-isabell, mit länglichem Knöllchen. Einige $\mathrm{cm}$ lang und $5 \mathrm{~mm}$ breit (nicht beim Knöllchen gemessen), bisweilen verbogen. I. zuerst dunkelbraun-violett, daan dunkelbraun. Sp. $10 \times 5.5$, mandelförmig, warzig. Viel kleiner als Langes und Mosers Pilze.

H. tortuosa Fr. - 21.8. - H. bis 9 breit, schokoladenbraun, trocken ockerbraun. F. blass violettlich, silberig faserig-gestreift, $10 \times 8$, auffallend verdreht. L. purpurzimt, rötend. Sp. $8-10 \times 4.5-$ 5.5, mandelförmig.

H. erythrina Fr. ss. Hry - 24.8. - Sp. subglobar $7.5-9 \times 5.5$.

H. erythrina Fr. ss. Rick. - 10.7. - Frühlingsform. Sp. $7-8 \times 6$, subglobar.
H. lanigera Fr. -20.8 .

H. armeniaca Schff. -21.8 .

H. damascena Fr. -23.8 .

H. rigens Pers. -21.8 .

$H$. bovina Fr. ss. Langei -31.8 .

H. glandicolor Fr. -15.8 .

H. punctata Pers. - 28.8. - Dieser Pilz ist erheblich grösser, als der, den ich 52 in Matoniemi fand. Siehe Kap. 4 . H. 4 breit. F. $10 \times 8$, keulig. Gürtel sehr flüchtig. Bei älteren Ex. ganz verschwunden. Sp. $10 \times 5.5$, mandelförmig, punktiert. Lit. über diesen Pilz dürftig. Kollektivart?

H. brunnea Fr. - 19.8 .

H. brunneofulva Fr. ss. OvS. - 2.9. - Lit. widersprechend. Ich verstehe unter ihm folgenden Pilz: H. bis 5 breit, glockig, dann mit schwachem Buckel, ausgebreitet, nass dunkelumbra, trocken dunkelrostbraun, glänzend. Schwach hygrophan. Haut abziehbar. F. bis $6 \times 10$, unten etwas dicker, Farbe des H., aber unten ganz blass. Ringzone bräunlich. L. dunkelrostbraun, breit. Sp. 8.5$9 \times 6$, mandelförmig, warzig. Mild.

H. hemitricha Fr. - 19.8.

H. flexipes Pers. -26.8.

H. paleacea Weinm. -17.8 .

$H$. rigida Scop. ss. Langei -22.8 .

$H$. balaustina Fr. ss. Rick. - 14.8. - H. 4 breit, zuerst bucklig, nass rotbraun, trocken rostfarben, durch eingewachsene Fasern gestreift. F. $5 \times 3-10$ (Knolle), braun, faserig gestreift. L. zimtrot. Sp. $6.5-7 \times 5.5$.

H. jubarina Fr. -25.7 . - H. 4 breit, glockig gebuckelt, dunkel zimt-rostbraun, schwach hygrophan. F. $6 \times 6$, Farbe des H., unten blass. L. breit, ziemlich entfernt. Sp. punktiert rauh, mandelförmig c. $8 \times 4.5$. Im Mischwalde. Nur ein Exemplar.

H. rubricosa Fr. -19.8 .

H. uracea Fr. ss, Langei -29.8 .

$H$. scanders Fr. -27.8 .

H. obtusa Fr. - 14.8 .

H. fulvescens Fr. - 14.8. - H. 1.5 breit, rotbraun, mit spitzem, sehr dunkelm Buckel. Schwach hygrophan. F. $4 \times 3$ blass, wird rostbraun. L. zimt-orangebraun. Sp. beinahe glatt, $11 \times 6$ etc.

$H$. decipiens Fr. ss. Langei -30.8 .

H. fasciata Fr. -15.8

H. Junghuhnii Fr. -16.8 .

$H$. acuta Fr. -15.8 .

Myxacium delibutum Fr. - 21.8.

M. collinitum Pers. - 29.7 .

$M$. stillatitiun Fr. -27.8 . - H. bis 5 breit, graulederfarben. F. violett. L. zuerst violettlich. Sp. $13 \times 8$ etc. Grosse, fast kugelige Cyst. auf der Schneide.

M. mucifluum Fr. -1 t.8.

M. elatior Fr. -20.8 .

M. mucosum Bull. - 15.8 .

$M$. vibratile $\mathrm{Fr} .-14.8$.

M. pluvium Fr. -26.8 .

Phlegmacium largum Fr. ss. Kühn. et Romagn. 14.8. - H. bis 9 breit, blassviolett. F. sehr dick, kahl, violett. Knolle bis 30 breit. L. zuerst violettlich. Sp. $11 \times 5.5$, mandelförmig, ziemlich glatt. Fl. violettlich, bei Behandlung mit Ammoniak nicht gilbend.

P. pseudocrassum Joss. (Hebeloma crassum Rick.) 14.8. - H. 7 breit, filzig, ockerrot. F. bis $10 \times$ 20-40 (Keule), weiss. L. holzfarben, abgerundet 
angewachsen, dicht. Sp. 7-8 $\times 4$, ziemlich glatt. Zahllose lange und schmale Gyst. Ohne Geruch und Geschmack.

P. crassum Fr. ss. Bres. -15.8 . - H. $6-7$ breit, derb, zimtfarben, nur am Rande etwas klebrig. F. $6-10 \times 20$, oben ausgesprochen weiss-schuppig. L. holzfarben (ziemlich blass). Sp. $10-11 \times$ 5.5, eiförmig bis elliptisch, körnigrauh. Keine Gyst. Fl. bleich, wässerig, durch Ammoniak gelb. Ziemlich geruchlos. Mischwald.

P. multiforme Fr. Unterart melliolens J. Schaeff. 30.8. - Sulfoformolreaction blaugrün.

P. aurantiacum Moser (napus Vel. non Fr.) - 29.7. Gehört zur multiforme-Gruppe. H. 5 breit, matt, ziegel- bis orangerot. Rand lange eingerollt. F. weisslich-gelblich, $5 \times 40$, L. blasstonfarben. Sp. etwa $7 \times 4$. Mischwald.

$P$. serarium Fr. ss. Rick. - 26.8. - H. bis 7 breit, orangefuchsig. Rand blassfaserig. F. bis $8 \times 15$, weiss, unten verdickt. L. zimtfalb. Sp. $7-8 \times 3$. Fast mild. Geruchlos. Nadelwald.

P. turmale Fr. - 20.8 .

$P$. triumphans Fr. - Hauptform. - 25.8.

$P$. triumphans Fr. f. minor Hry -17.8 .

P. subtriumphans Hry - 17.6. - Im Gegensatz zu $P$. triumphans $\mathrm{Fl}$. nicht gilbend bei Behandulng mit $\mathrm{KOH}$. Sp. etwas kleiner, etwa $11 \times 5.5$.

P. cliduchum Fr. ss. Konr. et Maubl. non Rick. 25.8.

P. cephalixum Hry non Secr. -21.8

$P$. decolorans Pers. ss. Cke -16.8 .

P. decoloratus Fr. ss. Langei -15.8 .

P. fulmineum $\mathrm{Fr} .-20.8$.

$P$. subtortum Fr. -22.8 . - H. bis 10 breit, gelbbraun mit Olivton. F. bis $10 \times 15-25$ (Keule), blass. L. sehr breit, olivgrau. Sp. $7-8 \times 5.5-6$, warzig. Enorme Cyst. Fl. schmutzig. Schwach bitter. Nadelwald.

$P$. olivascens Batsch ss. Langei - 30.8. - Etwas hygrophan. H. bis 4 breit, oliv, trocken gelblich schmutzig. F. bis $9 \times 10$, oben schmäler, blass, dunkler gestreift, oben angedeutet bläulich. L. oliv, schliesslich rostfarben. Sp. 7.5-8 $\times 5.5$, dickwandig, schwach körnig. Fl. blass, scharf. - Mosers olivascens $=$ olivascens ss. Bataille.

Conocybe tenera Schff. var. subovalis Kühn. - 1.7. Stark entwickelte Knolle.

C. tenera Schff. ss. Kühn. - 7.7. - Dauernd durchscheinend gerieft.

C. tenera Schff. var. semiglobata Kühn. - 15.7. Kaum gerieft.

Bolbitius fragilis L. - 30.6. - Kurzsporige Var. von $B$. vitellinus. Sp. $11 \times 7.5$. Nur Hutrand gerieft. Auf verfaultem Stroh.

Agrocybe dura Bolt. - 16.7. - Wie A. praecox, aber ohne Mehlgeruch und Mehlgeschmack. L. blass, dann viol.-grau, schliesslich kaffeebraun. Sp. gross und breit, $12 \times 8$ etc. Bitter. Abgemähtes Kleefeld.

A. praecox Pers. - 16.7 .

A. paludosa Lange (sphaleromorpha Bull.) - 20.7. Wie $A$. praecox. Unterschiede: kastanienbräunlich, hygrophan (trocken lederfarben), kleiner, mit relativ langem $\mathrm{F}$. Sp. dickwandiger, $8 \times 5$ etc. Sumpfgebiet.

A. semiorbicularis Fr. - 16.7. - H. 1-2 breit, glockenförmig, nicht hygrophan, gelblich. Mitte bräunlich. Ungerieft. F. $3-4 \times 1-2$, strohfar- ben, kahl, zäh. L. milchkaffeebraun, breit, angewachsen. Sp. dickwandig, $13-15 \times 8-10$, eiförmig. Cyst. flaschenförmig. Mehlgeruch. Abgemähtes Kleefeld. Vermutlich $=A$. pediades Fr.

Pholiota heteroclita Fr. - 26.8. - H. bis 7 breit, gelbbraun, durch kleine, dunkle, eingewachsene Schüppchen bunt. F. von unten innen bräunend. Ring rudimentär. Sp. $6-8 \times 4-5$, braun. Stark riechend. Auf und neben Birken.

P. flammans Fr. -17.8 .

P. lubrica Pers. - 25.8. - H. bis 6 breit, fuchsigfalb, sehr klebrig, zuerst glockig, dann niedergedrückt. F. $5 \times 10$, etwa gleichfarbig, faserig gestreift. L. tonfarben-oliv, breit. Sp. $5.5-6.5 \times$ 3.5-4.25, schmutzig. Kein Rettichgeruch (eher Jodoform?). Holzplatz, am Waldrande. Gleicht sehr einer Hebeloma.

P. spumosa Fr. - 14.8 .

$P$. astragalina Fr. -22.8

$P$. alnicola $\mathrm{Fr} .-28.8$

P. scamba Fr. - 25.8. - H. 1.5 breit, klebrig. Farbe von $P$. spumosa $=$ gelblich, mit fuchsiger Mitte. F. 2-3 $\times 3$, oben gelblich, unten fuchsig, faserschuppig, verbogen. L. grüngelb, ins Olivfuchsige übergehend. Sp. gewöhnlich $8-9 \times 5$, eiförmig bis oval. Fl. gelb. Auf verfaulter Fichtenborke. Vielleicht nicht identisch mit dem Funde von 53. Siehe Kap. 5. In der Lit. ist darauf hingewiesen worden, dass der Name für zwei versch. Pilze angewendet wird - eine Pholiota und eine Ripartites.

Kuehneromyces mutabilis Schff. - 25.6.

Naematoloma capnoides Fr. - 20.8 .

$\mathcal{N}$. sublateritium Fr. -17.8 .

N. fasciculare Huds. - 25.6.

Stropharia squamosa Pers. - 1.7. - Nur zwei kleine Ex. H. 2 breit, ockergelb bis fuchsig mit concentrischen Schüppchen. F. $3 \times 3$, oben weiss und unter dem Ringe leicht schuppig-filzig. L, olivpurpurn. Sp. dickwandig, $12-16 \times 7-8$, violett. Auf Holzstückchen im Walde.

S. stercoraria Fr. - 19.7 .

S. semiglobata Batsch -4.7 .

Psilocybe uda Pers. - 16.7.

P. merdaria Fr. -23.6 .

P. coprophila Bull. - 11.7.

$P$. semilanceata Fr. - 16.7. - H. bis 2 breit, dauernd spitzgebuckelt, braungelb. Am Rande häufig mit einer dunkleren, durchwässerten Zone. F. blass, $5 \times 1$ etc. L. mit weissen Flocken. Sp. ziemlich hell, durchsichtig, ei- bis subcitronförmig, bis $16 \times 10$. Abgemähtes Kleefeld.

Deconica atrorufa Schff. - 17.7 .

D. rhombispora Britz. - 21.8. - H. 1-1.5 breit, braun, stumpf gebuckelt. F. bräunlich, $3 \times 1$. L. schokoladenbraun mit hellerer Schneide. Sp. citronförmig bis geradezu rhombisch., $6 \times 5$ etc. Auf Blättern.

Panaeolus papilonaceus Bull. - 29.6. - H. bis 3 breit, weissblass, im Alter rissig-schuppig. F. gleichfarbig, oben weissstaubig. L. breit, mit weisslicher Schneide. Sp. $12-14 \times 8.5-10$, undurchsichtig, braunschwarz. Weideland.

$P$. campanulatus L. -1.7 .

P. sphinctrinus Fr. -22.7 .

$P$. acuminatus Fr. -19.7 .

P. subbalteatus $\mathrm{Bk}$, et $\mathrm{Br}$. -23.8 . 
P. fimicola Fr. var. ater Lange. -6.7 .

Panaeolina foenisecii Pers. - 15.7.

Psathyrella Candolleana Fr. - 2.7.

P. spadicea Schff. -24.8 . - H. bis 7 breit, hygrophan, schokoladenbraun, trocken falb. F. weiss, häufig gekrümmt. L. fast frei, zuerst blass, dann rötlich und schliesslich sckokoladenbraun. Sp. braun, 7-9 $\times 4-5.5$, elliptisch. Büschelig an und bei Stämmen.

P. gyroflexa Fr. -26.7 . - H. 1.5-2.5 breit, beinahe kastanienbraun, bis zur Hälfte gerieft. Trocken beinahe lederfarben und runzelig. F. weiss, $4 \times 2$, kahl. Basis dicker, weisszottig. L. ausgebuchtet angewachsen, schmutzigviolett, mitteldicht. Sp. $7.5 \times 4.5$. Staub braun-sepia. Im Walde, auf Blättern. Vielleicht nur eine Var. von $P$. oblusata?

P. subnuda Karst. - 22.8.

P. obtusata Fr. -6.7 . - H. $2-5$ breit, honigbraun, ausbleichend, durchscheinend gerieft, etwas gerunzelt. F. $6-9 \times 2-4$, unten weisstriegelig. L. schokoladenbraun. Sp. $7.5 \times 5$, eiförmig, hellviolettbraun. Cyst. ballonförmig.

Coprinus domesticus Pers. - 30.6.

C. truncorum Schff. - 18.8. - Wie C. micaceus, aber Sp. nicht mitraförmig (d.h. in der Nähe der Basis am breitesten).

C. niveus Pers. - 14.8 .

C. plicatilis Curt. -16.7 .

Russula delica Fr. - 25.7.

$R$. densifolia Secr. -14.8 .

R. adusta Pers. - 13.7

$R$. ochroleuca Pers. -11.7 .

R. foetens Fr, -18.7 .

$R$. consobrina Fr. -17.8

R. lilacea Quel. - 25.7.

$R$. brunneo-violacea Crawsh. -25.7 .

R. heterophylla Fr. -25.7 .

$R$. vesca Fr. - 8.7.

R. claroflava Grove - 18.7 .

$R$. decolorans Fr. - 14.8 .

$R$. inosa Lindbl. -21.8 .

$R$. xerampelina Schff. var. erythropus (Pelt.) M. et. Z. 5.7.

R. xerampelina Schff. var, fusca M. et Z. -24.7 .

$R$. xerampelina Schff. var. graveolens Rom. ss. Langei -14.7 .

$R$. xerampelina Schff. var. olivascens M. et Z. - 22.8.

$R$. xerampelina Schff. var. pascua Moeller et J. Schaeff. - 22.7. - Gelb. Klein.

$R$. xerampelina Schff. var. pseudomelliolens Singer? 11.7 - Ziegelrot.

R. xerampelina Schff. var. typica -27.8 .

$R$. venosa Vel. ss. Melz. - 19.7

R. puellaris Fr. -18.7 .

R. lutea Huds. - 18.7.

R. chamaeleontina Fr. ss. Lange -5.7 .

$R$. aeruginea Lindbl. -6.7

R. mollis Quel. ss. Langei - 30.7. H. bis 6 breit, olivbräunlich. Haut weit abziehbar. Rand im Alter gefurcht. F, ganz weiss. Sp. $8.5-10 \times 7-8$, ockergelb, mit groben isolierten Warzen. Ohne Geruch und Geschmack.

R. paludosa Britz. - 26.7.

R. Velenouskyi M. et Z. -19.7 .

R. pseudointegra Arn. et Goris. - 24.7. - H. 6 breit, zinnober bis rosenrot. Mitte ausbleichend (gil- bend). Klebrig, aber bald trocken. Rand gerieft. Haut bis zum Centrum abziehbar. F. weiss, schwammig, runzelig. L. werden sattocker, breit, bisweilen bewimpert. Sp. F-G. Orn. 5. Grösse etwa $8 \times 7$. Mild, langsam etwas bitter. Sieht wie $R$. emetica aus.

R. olivacea (Schff.) Fr. -20.7 .

$R$. curtipes Moell. et Schff. - 27.8. - H. bis 12 breit, purpurrot, in der Mitte ocker. Haut schwer abziehbar. F. weiss, kurz und meist dick, bei Berührung bräunend. L. vorne sehr breit, hinten etwas abgerundet, hellocker. Sp. F. Orn. 3. Grösse $7.5 \times 6.5$. Steht $R$. Romellii sehr nahe.

$R$. alutacea Fr. ss. Melz. et Zvara - 7.7.

$R$. emetica Schff. var. typica -18.7 .

$R$. emetica Schff. var silvestris (Sing.) f. pithyophila Romagn. - 23.7. - Wie Hauptform, aber klein und weiss unter der Huthaut. Sp. $9-12 \times 6.5-8$.

R. violacea Quel. -2.7 . $-\mathrm{H}$. bis 5 breit, dunkelpurpurn, zum Rande zu heller, klebrig. Rand schwach gerieft. Haut zur Hälfte abziehbar. F. adrig, weiss, bisweilen gilbend. L. beinahe weiss, gleichlang. Sp. C. Orn. 5. Grösse $8 \times 7$ etc. Scharf. Keine Reaktion auf Ammoniak. Sehr gebrechlich.

$R$. zonatula Ebbes, et J. Schaeff. - 26.7. - Sehr gebrechlich. H. bis 6 breit, violettlich-fleischrot. Mitte bisweilen bräunlich. Sehr klebrig. Rand stark gerieft. Haut abziehbar. F. weiss, adrig, bis $6 \times 12$. L. weiss bis crème, vorne am breitesten. Sp. crème, mit groben isolierten Warzen, $8-10 \times$ 7-9. Nur L. schärflich.

R. sanguinea Bull. var. rosacea Langei (rosacea (Pers.) Fr. ss. Bres.) - 14.8. - L. im Gegensatz zur Hauptform nicht herablaufend. Verdient kaum als Art oder Var. angesprochen zu werden.

R. Queletii Fr. -20.8 .

Lactarius torminosus Schff. - 16.8 .

L. pubescens Fr. ss. Rick., Pears. - 28.8. - H. bis 5 breit, trocken, ungezont, weiss bis weissgelblich. Rand bärtig. F. $2-3 \times 15$. L. schmal, dicht, blass, mit kaum sichtbarem fleischrötlichem Ton. Sp. bis $7 \times 5$, gewöhnlich kleiner. Milch weiss, scharf. Auf einer mit Birken bewachsenen Wiese.

L. scrobiculatus Scop. -21.8 .

L. repraesentaneus Britz. - 19.8 .

L. deliciosus L. -24.7 .

L. necator Pers. -30.7

L. vietus $\mathrm{Fr} .-19.8$.

L. trivialis Fr, -14.8 .

L. picinus Fr, -17.8 .

L. glyciosmus Fr. -20.8 .

L. griseus Peck - 20.8. - H. bis 2 breit, mit kleiner Papille, braun resp. rauchgrau, ungerieft, schwach filzig. F. $2 \times 3$, heller als $\mathrm{H}$. L. schmutzigcrème. Sp. $7-8 \times 5.5-6.5$. Warzen in einem unvollständigen Netz verbunden. Zuerst mild, dann etwas scharf. Im Mischwalde, auf toten Ästen (Eller?). KOH-Reaktion auf dem $\mathrm{H}$. rostigorange.

L. flexuosus Fr. - 10.7.

L. spinosulus Quel. - 27.8. - H. bis 5 breit, blasskarminrosa, mit aufgerichteten Schüppchen (nur im Centrum liegend). F. gleichfarbig, nur etwas heller, bis $6 \times 8$. L. werden rötlich. Sp. etwa $7.5-8 \times 6.5-7$, crème. Milch weiss, mild, später scharf.

L. mitissimus Fr. -18.7 . 
L. cremor Fr. ss. Karst., Rick. - 18.8. - H. bis 5 breit, braungelb mit Orangeton, flachgenabelt, mit breit herabgebogenem, wellig-rippig-gerieftem Rand. F. etwa gleichfarbig, vielleicht etwas blasser, bis $4 \times 8$. L. zuerst blass, schliesslich fleischrot, undicht, breit. Sp. 7.5-9 9 7-8 mit groben St, die zum grossen Teil mit einander verbunden sind. Mild. Strittige Art.

L. obscuratus Lasch ss. Pears. - 8.7.

L. helvus Fr. - 20.8 .

L. camphoratus Bull. - 29.7 .

L. rufus Scop. - 14.8 .

L. subdulcis Bull. ss. restr. -8.7 .

L. theiogalus Fr. ss. Rick (decipiens Quel.) - 20. 8. $H$. bis 5 breit, rosaledergelb, ungezont, zart runzelig. F. gleichfarbig, unten purpurbräunlich, wellig-uneben. L. sehr blass fleischfarben, dünn, dicht. Sp. $8 \times 6$ etc. netzig. Milch langsam gilbend.

L. tabidus Fr. ss. Konr. (theiogalus (Bull.) Fr.) 29.8. - Hygrophan. H. bis 4 breit, rötlichblass, mit Papille, durchscheinend gerieft. F. bis $5 \times 5$, rostrot, unten weissfilzig. L. Farbe des H. Sp. 7-9 $\times 5.5-7$ mit meist isolierten Warzen. Milch weiss, gilbend. Mild.

Cantharellus cibarius Fr. - 3.7.

C. infundibuliformis Scop. -14.8 .

C. sp. -31.8 . - H. 2 breit, kreiselförmig mit derbem Buckel (in der Art von Camarophyllus pratensis), glatt, weiss bis sahnefarben, seidig. F. $2 \times 3$, verbogen, bisweilen kanalisiert, weiss. L. weiss, am Rande des H. gegabelt. Sp. $9-10 \times$ 3-4, cylindrisch. Im Freien, auf einer Viehweide.

Craterellus cornucopoides L. - 17.8.

Lycoperdon echinatum Pers. - 19.8.

L. umbrinum Pers. - 16.8 .

L. gemmatum Batsch - 21.7.

L. piriforme Schff. -29.7 .

L. papillatum Schff. - 17.7. - Beinahe rund, $2 \mathrm{~cm}$ im Diam., graubräunlich, mit etwa $1 \mathrm{~mm}$ langen, pyramidenförmigen, braunen, bisweilen zusammenneigenden St. Flachere St. untermischt. Nadelwald.

Bovista plumbea Pers. - 14.8 .

B. nigrescens Pers. - 23.6.

Geaster Bryantii Berk. - 22.6. - Sehr schöne vertrocknete Ex. von 1953. Innenkörper schwarzbläulich, bis $20 \mathrm{~mm}$ breit. Mündung lang, kegelförmig, gefurchtgefaltet. Kragen um den Stiel, der nicht kammförmig gefurcht ist. Exoperidie mit 7-9 Lappen, weisslich-ockerlich, bis 6 breit. Auf Ameisenhaufen, im Nadelwalde.

Tremella fimbriata Pers. - 16.8. - Olivschwarz, aus flatterigen, runzeligen, am Grunde verbundenen Lappen bestehend. Rand der Lappen wellig. Fk. $2 \mathrm{~cm}$ hoch und $3 \mathrm{~cm}$ breit. Sp. $7 \times 5.5$. Auf totem Birkenstamm. Exs. leider verkohlt.

Dacryomyces stillatus Nees - 24.6.

D. sp. - 27.7. - Rot. Einige $\mathrm{mm}$ im Diam. Ohne Fuss. Sp. grobwarzig, $20 \times 20$.

Calocera viscosa Pers. - 22.7.

C. furcata Fr. - 14.8 .

Clavaria virescens Gramb. (cyanescens Romell) - 16.8 . - Der ganze Pilz mit Ausnahme des unbedeuten- den, weissen Strunkes grün. Sp. $7 \times 4$, ocker, rauhlich. Vielleicht eine Var. von C. abietina Pers.

C. Invalii Cott. et Wakef. - 16.8. - Vermutlich = C. abietina Fr. non Pers. Spitzen nicht grün. Sp. $7-9 \times 4-5$, kernförmig, hellocker, rauh. Typisch für den Norden Europas.

C. ligula Schff. - 17.8.

Stereum hirsutum Willd. - 15.7.

S. sanguinolentum Schw. -17.8 .

S. purpureum Pers. -22.7 .

Hydnum repandum L. -29.7.

H. rufescens Pers. - 17.8 .

Calodon sulphureum Kalchbr. - 26.8.

C. cyathiforme Schff. -14.8 .

Phylacteria terrestris Ehrh. - 30.6 .

Polyporus ovinus Schff. -23.8 .

Melanopus varius Pers. (picipes Fr.) - 30.7. - H. gelbbraun, fast faserstreifig, fächerförmig, kahl, bis 12 breit. F. schwarz. R. unregelmässig am F. abgesetzt. Sp. $6-8 \times 3-3.5$. Süsslicher Geruch. An Laubholz.

M. elegans Bull. - 16.8 .

M. melanopus Pers. -28.7

Leucoporus brumalis Pers. -25.6 .

Leptoporus albidus Trog. Schaeff. - 30.7.

L. amorphus Fr. - 25.8. - H. bis 4 breit, reinweiss, fast häutig-dünn. R, blass, werden orangegelb. Sp. $3-3.5 \times 1-1.5$. Fl. zäh, weiss, bitter. Auf faulen Kiefern.

L. dichrous Fr. - 23.7. - Ohne F. Dachziegelig und zusammenfliessend. H. bis 4 breit, sehr dünn, flatterig, grubig, fein behaart. Farbe nicht feststellbar, da mit grünen Algen bewachsen. Rand weisszottig, scharf, steril. Trama (weiss), gelatinöses Subhymenium und Hymenium unter Lupe scharf getrennt. R. unregelmässig, weiss, mit rötlichem Schimmer. Sp. $3-4 \times 1.5-2$, verbogen., weisslich. Auf Holz, vermutlich Birke. L. adustus Willd. - 28.7.

Phaeolus rutilans Pers. - 23.7. - H. 3-6 breit, Bisweilen kissenförmig ( $2 \mathrm{~cm}$ dick), aber auch dünn, zimtgelb oder zimtfuchsig. Oberfäche anfangs filzig, dann uneben-faltig. Fl. dauernd weich. Sp. $3-5 \times 2-2.75$. Auf Birkenast.

Coriolus hirsutus Schrad. - 21.7.

C. versicolor $\mathrm{L}$. -21.6 .

C. unicolor Bull. -7.7 .

C. abietinus Dicks. -22.6 .

Lenzites betulina L. - 25.6.

L. sepiaria Wulf. -25.6 .

L. heteromorpha Fr. (Trametes h.) - H. 2-3 breit, weissblass. L. weiss, sehr breit. Sp. $11-13 \times 4-5$, bisweilen verbogen. Auf Fichtenstümpfen, dachziegelig.

Trametes cinnabarina Jacq. - 16.8 .

T. odorata Wulf. - 2.9 .

Ungulina fomentaria L. - 23.6.

$U$. marginata $\mathrm{Fr} .-23.6$.

$U$. resinosa $\mathrm{Fr} .-25.6$.

$U$. betulina Bull. -25.6 .

Ganoderma applanatum Pers. - 28.6.

Phellinus igniarius L. - 22.6.

Xanthochrous perennis L. -2.7 . 


\section{ASCOMYCETES.}

Cudonia confusa Bres. - 14.8.

Vibrissea truncorum Schw. - 24.6.

Apostemidium fiscelle Karst. - 22.6. - Exs. im Forstwissenschaftlichen Institut in Helsinki. 1-2 mm im Diam. Bläulichgraue Scheibchen mit Haaren (Lupe) besetzt. Ohne deutlichen F. Sp. $200 \times 1-2$ auch länger, farblos. Auf verfaultem Holz, im Wasser.

Mitrula phalloides Bull. (paludosa) - 25.6. $-2 \mathrm{~cm}$ hoch, keulenförmig, orange. Sp. farblos, 14$16 \times 3-4$, stumpf- spindelig.

Macropodia macropus Pers. - 25.7. - Schüsselförmig, 1-2 breit, mit scharfem, oft zerschliztem Rand, dünn, mit graubrauner Scheibe, aussen weissgrau, haarig und rauh. Lederig-gebrechlich. F. weissgrau, haarig, $3 \times 5$. Sp. spindelig, $22 \times 10$, beinahe glatt, mit einem grossen Öltropfen. Im Walde.

Otidea sp. 3. -26.8 . - Vielleicht $=0$. felina Pers., deren $\mathrm{Sp}$. aber 2-tropfig sein sollen. Bis $2 \mathrm{~cm}$ hoch, innen gelblich, aussen rehfarben, bis zum $2-3 \mathrm{~mm}$ langen, dunkleren $\mathrm{F}$. gespalten. Rand schwach eingerollt, etwas filzig (Lupe). Sp. eintropfig, $10-11 \times 5.5-6$. Im Moose des Nadelwaldes, rasig.

Aleuria aurantia Müll. - 29.8.

Plicaria badia Pers. - 23.6.

P. brunneoatra Desm. - 24.7. - Etwa 1 breit, schüsselförmig. Scheibe granzrandig und beinahe schwarz. Aussen dunkelumbrabraun. Sp. warzig, mit undeutlichen Öltropfen, $16.5-19 \times 8.5-9.5$. Lehmiger Grabenrand.

$P$. fimeti Fuck. Blieb zuerst unbestimmt und wurde von mir als $P$. sp. registriert. - 24.7. - Sieht einer kleinen $P$. badia ähnlich. Bis 2 breit. Sp. $19 \times 10$, glatt, ohne Öltropfen. Auf Mist.
Lachnea scutellata. L. $-4.7 .-2-8 \mathrm{~mm}$ breit, schüsselförmig-flach, rot, auf der Aussenseite behaart. Trocken verblassend und eingerollt. Sp. $20 \times 12$, rauhlich. Auf faulem Holz.

\section{d) Lappland.}

Ich verbrachte Anf. Aug. etwa 10 Tage in Nordnorwegen und Lappland, ohne mich daselbst besonders den Pilzen widmen zu können. Nur zweierlei fiel mir auf: der grosse Pilzreichtum und die Tatsache, dass Leccinum testaceoscabrum Secr. in Lappland weit oberhalb der Grenze für die gewöhnlichen Birkenarten in Symbiose mit der kriechenden Betula nana ganz vorzüglich gedeiht.

\section{e) Åland.}

Ich war daselbst in der Zeit zwischen dem 10 Sept. und 11 Okt. mit dem Zwecke meine Kenntnisse der Pilze Alands durch Beobachtungen der Herbstflora zu ergänzen. Entsprechend finden sich in der nun folgenden Liste nur solche Pilze, die ich 1948-50 auf Åland nicht fand. Alle Funde wurden auf der Insel Eckerö gemacht, mit Ausnahme derjenigen vom 10 September, die aus dem Park (Bot. Schutzgebiet von Mariehamn) und dem angrenzenden Nadelwald stammen.

\section{BASIDIOMYCETES.}

Leccinum aurantiacum Bull. - 10.9 .

L. rufescens. Secr. -10.9 .

L. leucophaeum Gilb. - 10.9 .

L. leucophaeum Gilb. var. coloratipes Sing. -3.10 .

Paxillus filamentosus Fr. - 11.9.

Hygrophorus piceae Kühn. - 11.9.

H. cossus Fr. - 11.9.

H. hypothejus Fr. -25.9 .

H. discoideus Pers. - 21.9. - H. bis 4 breit, fuchsigbraun. Mitte dunkler. F. bis $4 \times 8$, schleimig, blass, faserstreifig. Spitze weisslich. L. weiss, dann gelblich. Sp. $6-7 \times 3-4$. Nadelwald.

H. fuscoalbus Lasch - 19.9. - Nur ein kleines, ziemlich schlechtes Ex. H. 3 breit, oliv, niedergedrückt, Mitte dunkel gebuckelt. F. $10 \times 4$, weiss. L. weiss herablaufend. Sp. etwa $10 \times 7$. Vielleicht identisch mit $H$. mesotephrus Br. ss. Kühn. et Romagn. H. olivaceoalbus sehr nahestehend.

H. pustulatus Pers. - 1.10.

Camarophyllus russocoriaceus $\mathrm{Bk}$. et $\mathrm{Br} .-20.9$, $-\mathrm{H}$. bis 2.5 breit, elfenbeinweiss, hygrophan, wird weiss. Durchscheinend gerieft. F. verbogen, $3 \times 2$, weisslich. L. herablaufend. Sp. $7.5 \times 5$. Geruch nach Juchtenleder. Viehweide.
C. virgineus Wulf. -26.9 .

C. niveus Scop. - 14.9.

Hygrocybe nitrata Pers. - 16.9.

$H$. nigrescens Quel. - 10.9 .

H. punicea Fr. - 11.9. - H. 8 breit, granatrot. F. $5 \mathrm{~cm}$ lang und dick, nach unten verdünnt, faserig gestreift. Basis weiss. L. bauchig angewachsen. Sp. $8.5-11 \times 5-5.5$

H. psittacina Schff. -15.9 .

H. ceracea Wulf. - 12.9 .

H. citrina (Rea) var. glutinipes Lange - 11.9.

H. Reai Maire var. insipida Lange - 26.9.

H. coccinea Schff. -20.9 .

H. cantharellus Schw. - 14.9.

H. laeta Pers. - 13.9. - H. bis 3 breit, orangegelb, durchscheinend gerieft, sehr schleimig. F. bis $5 \times 6$, gleichfarbig. Spitze bisweilen grünlich. Sehr schlüpfrig. L. graulich, werden fleischfarben. Sp. $5.5-6 \times 4-4.5$.

Lyophyllum ozes Fr. ss. OvS. - 23.9. - H. bis 3 breit, schwarzbraun. F. bis $3 \times 3$, verbogen, faserig-längsrissig, blass. Spitze weissmehlig. L. grau, breit, beinahe frei. Sp. $5 \times 3$. Mehlgeruch. Entspricht genau Mosers Beschreibung mit Ausnahme der Sp. Grösse. Nicht identisch mit Kühn. et Romagn. Pilz. Eher $=$ L. baeospermum Romagn 
L. putidum Fr. - 3.10.

L. miserum Fr. ss. OvS. - 2.10. - H. bis 3 breit, deutlich gerieft, grau, flach, mit kleinem Buckel. F. bis $7 \times 2$, schwach gerieft. starr, grau, oben weissmehlig, unten weisszottig, nicht wurzelnd. L. grau, angewachsen. Sp. gewöhnlich $6.5 \times 3$. Mehlgeruch. Nadelwald. Literatur unklar und widersprechend.

L. infumatum Bres. - 6.10.

L. capniocephalum Bull. - 12.9. - H. bis 7 breit, russfarben. F. bis $5 \times 15$ (Knolle 20), graulich, dunkel gefasert. L. grau. Sp. $5-5.5 \times 3.5-4$. Riecht unangenehm. Überall schwärzend.

Clitocybe geotropa Fr. - 17.9. - H. bis 15 breit, lederfarben, trichterförmig, undeutlich gebuckelt. $\mathrm{F}$. bis $12 \times 30$, oben dünner. L. und $\mathrm{F}$. weisslich. Sp. $6-7 \times 5-5.5$. Waldwiese.

C. nebularis Batsch -10.9 .

C. inversa Scop. - 13.9.

C. inversa Scop. f. pallidispora OvS. - 22.9. - Robust, in der Mitte dunkelrot. Sp. $5-5.5 \times 3-3.5$, rauh, hyalin, schmutzig.

C. ericetorum Bull. - 13.9. - H. bis 4 breit, weiss/ lederfarben, zuerst genabelt, dann kreiselförmig, kahl. F. abwärts verjüngt, $3 \times 5$, gleichfarbıg. L. ungleich lang. Sp. zum Teil mit Füsschen, $4 \times 3$, auch ganz rund. Erinnert an eine Hygrocybe.

C. leucophylla Fr. (Omphalia l.) -20.9 . $-\mathrm{H}$. bis 3.5 breit, blassbräunlich, hygrophan, trocken weisslich, tief genabelt, Rand lange eingerollt. F. bis $4 \times 4-8$, breitgedrückt. L. weisslich, schmal. Sp. $6 \times 3$. Auf Nadeln.

C. ditopa Fr. -3.10 .

C. Langei Sing. - 9.10. - H. 2 breit, schmutzig grau, wird blass-graulich, flachgewölbt-niedergedrückt. F. $3 \times 3$, gleichfarbig. L. gleichfarbig. Sp. 5-5.5 $\times 3$, apiculus zugespitzt. Mehlgeruch.

C. diatreta $\mathrm{Fr} .-3.10$.

C. metachroa Fr. -2.10

C. brumalis Fr. -28.9 .

Laccaria laccata Scop. f. amethystina - 10.9.

Tricholomopsis decora Fr. - 13.9.

Collybia cirrhata Schum. var. typica R. Maire 24.9 .

C. tuberosa Bull. - 25.9.

C. peronata Bolt. -23.9 .

C. butyracea Bull. -20.9 .

C. prolixa Fl. Dan. ss. Moseri - 4.10. - H. bis 7 breit, braunrot, hochgewölbr. F. längsfaserig gestreift, bis $12 \times 10$, weiss, rotfleckig, wurzelnd. L. weisslich mit schwachem rötlichem Schimmer, gesägt, dicht. Sp. $5.5-6.5 \times 4-4.5$ mit rötlichem Schimmer, in der Form sehr unregelmässig. Geruch süsslich. Nadelwald.

C. erosa Fr. ss. OvS. - 23.9. - H. 2-4 breit, schmutzig graubraun mit dunklerem Buckel, ungerieft. F. 3-4 $32-4$, unten verdickt, sehr dunkel, faserig gestreift. L. weisslich, bauchig, ausgebuchtet angewachsen. Sp. warzig, $7 \times 5.5$. Mischwald. Steht C. tesquorum (siehe Kap. 5) sehr nahe.

C. tylicolor Fr. ss. Lange - 23.9. - H. bis 1.5 breit, gebuckelt, bräunlichgrau (etwas heller als Langes Pilz). F. $2 \times 1$, grau, glatt. L. weisslich, ausgerandet. Sp. stacheligwarzig, $7 \times 5.5$.

Omphaliella rustica Fr. - 12.9. - Wenig geklärte Art. Vielleicht identisch mit O. muralis Sow., Cantharellus cupulatus Fr. und Agaricus helvelloides Bull. H. bis 1.5 breit, graubraun, gerieft, genabelt.
Rand konvex, nach unten gebogen. F. bis $8 \times 1$, faserig gestreift, Farbe des H. L. hell graubraun, bisweilen halbiert, auch ästig-gabelig. Sp. 7.5$8 \times 5-5.5$.

Tricholoma pessundatum Fr. - 13.9. - H. sehr gross, rotbraun, nicht faserstreifig. F. blass, oben nicht abgegrenzt weissmehlig (im Gegensatz zu $T$. albobrunneum). Sp. $3-4 \times 2-3$, mit einem Öltropfen. Mehlgeruch und Mehlgeschmack.

T. tridentinum Sing. ( $T$. pessundatum Bres. non Fr.) 26.9. - Ähnlich der Friesschen Art. H. 4-7 breit, kastanienbraun. F. $4-5 \times 10-15$, weiss, abwärts bräunlich. L. weisslich, bisweilen rostfleckig. Sp. $5.5-6 \times 3-3.5$.

T. vaccinum Pers. -19.9 .

T. viridilutescens Moser - 16.9.

T. fucatum Fr. - 9.10. - Nur ein Ex. H. 4 breit, schmutzigbraun in der Mitte, gelblicher am Rande, überall mit Olivton, durch dunkle radiale Fasern getigert. F. $4 \times 8$, gleichfarbig mit dunklen Fasern. L. weisslich. Sp. 5.5-7 $\times 5-6$. Geruch und Geschmack von Mehl. Von T. sejunctum und viridilutescens schwer zu trennen.

T. sulphureum Bull. - 16.9. - In allen Teilen schwefelgelb. H. bis 5 breit, wird fuchsig. F. bis $10 \times 10$, fuchsig-faserig. Sp. $9 \times 5.5$ etc. Stinkt.

T. portentosum Fr. -28.9 .

Cantharellula umbonata Gmel. - 15.9.

Leucopaxillus sp. - 17.9. - H. bis über $10 \mathrm{~cm}$ breit, crèmefarben, glatt, Mitte gewölbt (hier bis $3 \mathrm{~cm}$ dick, Rand aufspaltend. F. bis $10 \times 15-20$, crèmefarben, kahl, häufig excentrisch, verbogen. L. gleichfarbig, ungezähnt, breit, schwach herablaufend. Sp. weiss, $8-8.5 \times 5-5.5$ (Vergrösserung $\times 1350)$, feinwarzig, beinahe in der Art der Russulae. Fl, riecht unangenehm und wird schnell beinahe holzhart. Auf und neben toter Birke. Nachdem alle Versuche den Pilz einwandfrei zu bestimmen bei uns missglückt waren, hatte Prof. V. Kuja la die Freundlichkeit ein Ex. Dr. Seth Lun dell-Upsala zu schicken. Aus dessen Gutachten geht klar hervor, dass es sich hier um einen mindestens in Europa bisher unbekannten Leucopaxillus der konfusen Gruppe L. albissimus (Peck) Sing. handelt.

Melanoleuca graminicola Vel. ss. OvS. - 15.9. - H. bis 4 breit, schwarzgrau, trocken grau. F. bis $5 \times 5$, grau. L. ohne Cyst. und grau, nicht weiss wie bei Kühn. angegeben. Sp. $7-8 \times 5-6$, ziemlich kurz.

M. stridula Fr. - 24.9. (auch in Lohja 8.9. 54). H. bis 4 breit, dunkelgraubraun, kahl, mit kleinem Buckel. F, $8 \times 5$ (oben), unten knollig, dunkel faserig gestreift, unten weissfilzig. L. sehr dicht. Sp. warzig, $8 \times 5.5$. Sieht wie eine Collybia aus.

M. oreina Fr. - 15.9. - H. bis 4 breit, umbrabraun, nicht hygrophan, gebuckelt. F. $3 \times 4$, weisslich, leicht knollig. L. weiss, fast frei. Sp. $6.5-7.5 \times$ 4.5-5. Cyst. reichlich. Fl. im F. weiss.

$M$. brevipes Bull. ( $M$. grammopodia Bull. var. subbrevipes Metr.) - Wie M. grammopodia, aber L. graulich und Fl. im F. bräunlich.

M. grammopodia Bull. - 14.9. - H. bis 10 breit, graubraun mit bleibendem dunklerem Buckel. F. $5 \times 10$, faserig gestreift, keulig. Fl. im F. weiss. L. weiss. Sp. c. $9 \times 5.5$, warzig. Cyst. mit dünnem Schnabel. Starker Geruch.

M. melaleuca Pers. - 12.9. 
$M$. amica Fr. - 2.10. - Wohl nur eine der vielen Var. von $M$. melaleuca. Knolle. Sp. etwa $8.5 \times$ 5.5-6, also breit.

M. turrita Fr. - 12.9. - H. bis 8 breit, schwarzgrau, alt kahl. F. bis $10 \times 10$, unten verdickt (15), graubraun, dunkler faserig gestreift. L. grau. Sp. wie gewöhnlich bei den Melanoleucae. Cyst. spindelig, aber auch schopfig. Fl. grau. NB. Iche habe die von mir bemerkten Subarten, Varianten und Formen der Vollständigkeit wegen notiert, bin aber der Meinung, dass die Zergliederung der Melanoleuca-Gruppe erheblich zu weit geht.

Pleurotus tephrotrichus Fr. (corticatus Fr. var. tephrotrichus $=($ nach Pilàt $)$ dryinus Pers.) -15.9 . - H. $12 \times$ $8 \times 2$, muschelförmig, auf weisslichem Grunde dunkler beschuppt. F. excentrisch $2 \times 20$, mit sichtbarem Ring oder ohne solchen, schwach filzig. L. weiss, herablaufend, nur selten anastomosierend oder gegabelt. Sp. $9-12(-14) \times 4$ $-5(-5.5)$, cylindrisch, mit oder ohne Tropfen. Gilbend. Süsslicher Geruch. Auf Fichtenstamm. Am 8.10. auf Birke.

Panellus mitis Pers. - 1.10.

Macrocystidia cucumis Pers. - 6.10. - H. 1-3 breit, dunkelbraun, trocken ledergelb, konisch bis glockenförmig, nur nass durchscheinend gerieft. F. $2-4 \times 1-2$, rotbraun, bereift. L. blass, breit, frei. Sp. $8-9 \times 3-3.5$, hellrosa. Riesige lanzenförmige Cyst. Gurkengeruch. Die früher in Mustila gefundenen Ex. waren viel grösser.

Marasmius epiphyllus Fr. - 26.9. - H. bis 7 breit, weiss, runzelig. F. $2 \times 0.5$, oben weiss, unten braun, unter Lupe sammetig. L. 8-10 Stück. Sp. 8.5-10×3-4, spulenförmig. Auf Blättern.

Mycena maculata Karst. - 13.9. - H. bis 4 breit, graubraun, stark gerieft. F. graubraun, oben heller, gewöhnlich $6 \times 3$, zäh. L. grau, Schneide heller, angeblich im Alter rötend. Sp. $7-8(-9)$ $\times 4.5-5(-6)$. Geruch schwach alkalisch. Auf Strünken.

M. parabolica Fr. Vielleicht identisch mit $M$. maculata Karst. - 3.10.

M. epipterygia Scop. - 12.9.

M. alcalina Fr. f. chlorinella Lange - 27.9.

M. filopes Bull. ss. Schroet., Kühn. (vitilis (Fr.) Quel.) - 20.9. - H. bis 1.5 breit, graubraun, kegelig-stumpf, oft gebuckelt, gerieft. F. graubraun, fädig, zäh, kahl, glänzend, $8 \times 1$ etc. Spitze weisslich. L. weisslich. Sp. $9.5-10.5 \times$ 5.5-6. Keine Bürstencyst. Auf vergrabenem Holz.

M. olivascens Quel. ss. Oort. - 9.10.

M. zephirus Fr. - 21.9. - H. bis 4 breit, fleischbräunlich (Scheitel wird bräunlich bis rotbraun). Braunfleckig, runzelig-riefelig. F. bis $8 \times 5$, röhrig, Farbe des $H$., weiss überfasert. L. werden rostfleckig. Sp. $10-11 \times 5-5.5$. Sehr gebrechlich. Nadelwald.

$M$. aetites Fr. - 29.9 .

$M$. alcalina Fr. -30.9 .

M. flavoalba Fr. - 11.9.

Baeospora myosura Fr. - 6.10. - H. 1.5 breit, honigbraun. F. bis $3 \times 1$, bräunlich, oben verdickt, weissmehlig. L. weiss, frei. Sp. $4-4.5 \times 2.5-3$, schwach amyloid. Auf Zapfen, wenn scheinbar auf dem Erdboden, dann meist oder immer auf Resten von ganz unkenntlich gewordenen Zapfen. Lepista sordida Fr. -7.10 .
L. nuda Bull. - 24.9.

L. caespitosa Bres. - 27.9. - Eine im Freien wachsende Var. von L. panaeola Fr. H. bis 8 breit, tonockerbraun, mit eingebogenem Rand. F. $4 \times 10-15$, häufig excentrisch, blass. L. Farbe des H., aber blasser, leicht abtrennbar. Sp. $4.5-5.5 \times 2.75-3.5$, en masse rötlich. Geschmack sauer. Geruch etwas mehlartig, aber nicht angenehm. Büschelig. Viehweide.

Rhodophyllus speculus Fr. ss. Kühn. et Romagn. 17.9 .

R. staurosporus Lange var. Rickeni Romagn. - 26.9. - F. unten nicht verdickt. Schwach hygrophan. Kein Mehlgeruch und Mehlgeschmack. Sumpf.

R. mammosus (Fr.) Quel. ss. Konr. et Maubl. - 20.9.

$R$. clandestinus Fr. ss. Lange -25.9 . - H. bis 3 breit, beinahe schwarz, trocken blasser, gebuckelt. F. $4 \times 2$, gleichfarbig. L. ziemlich dick und entfernt. Sp. im allgem. ziemlich regelmässig $8 \times 8$, $9 \times 7$ etc. im Gegensatz zu den mehr ovalen des $R$. papillatus. Keine Randcyst.

R. junceus Fr. -13.9 .

R. icterinus Fr. -12.9 .

$R$. infulus Fr. -14.9 .

R. limosus Fr. -28.9 .

$R$. sarcitus Fr. -20.9 . - H. bis 3 breit, russigbraun, hygrophan, trocken dunkellederfarben, niedergedrückt, gerieft. F. $3 \times 2$, Farbe des H., faserig, kahl. L. hellbraun, mit sterilem, dunklerem (braunem) Rand. Sp. 8-10×6-7, sehr unregelmässig. Vielleicht $=R$. sarcitulus var. spurcifolius Kühn.

R. carneoalbus With. -13.9 .

R. sericellus Fr. -27.9 .

R. undatus Fr. - 11.9. - Úberall graubraun. H. 2 breit, trichterförmig, Rand durchscheinend gerieft. F. $2 \times 2$, blasser. Basis weissfilzig. L. herablaufend. Sp. $7 \times 5.5$ etc.

Pluteus sp. - 10.9. - Zwischen P. cervinus und petasatus stehend, vielleicht = petasatus Karst. Nur ein Ex. H. 4 breit. Rand undeutlich gerieft, olivbraun, grubig, ohne Schuppen. F. $5 \times 8$, Knolle c. 10. F. blass, braunfaserig gestreift. L. rosa, breit, beinahe frei. Sp. c. $5.5-6 \times 4-4.5$, bisweilen ganz rund. Huthaut nicht hymeniform. Cyst. überall gabelig, $40 \times 20$ etc. Rettichgeruch.

P. murinus Bres. -3.10 . $-\mathrm{H}$. bis 10 breit. Haut nicht hymeniform. Grau, radial gefasert, am Rande längs Faser aufspaltend und das weisse Fl. offenbarend. F. bis $10 \times 10$, weisslich, faserig gestreift, kahl, nur unten gepudert. L. breit, intensiv rosa. Sp. $8-9 \times 6-7$. Cyst. auf der Fläche der L. offenbar fehlend, auf der Schneide wenig hervortretend. Der über die Schneide herausragende Teil keulig und c. $6-8$ breit. Auf Nadelholzstamm.

P. leoninus Schff. - 13.9. - H. bis 4 breit, löwengelb. Rand gerieft. F. $6 \times 6$, heller, faserig gestreift. L. blass fleischfarben. Sp. etwa $7-8 \times 5.5$. Rettichgeruch. Auf Stümpfen.

Volvariella speciosa Fr. - 25.9.

V. sp. - 30.9. - Nur ein Ex., das bei der Untersuchung wenig gelitten hat. H. 3 breit, gelbrot, zu rötlichgelb ausblassend, glatt, nicht stark schleimig, niedergedrückt. F. $5 \times 4$, etwa Farbe des H. Weisse, nur schwach entwickelte Volva, vielleicht nur verdicktes weissfilziges Ende. Sp. 
$5.5-6 \times 5-5.5$, rötlich mit etwas bräunlichem Ton. Cyst. auf der Schneide sehr unregelmässig, keulen- resp. flaschenförmig, aber nie, wie bei $\mathrm{Pl}$. cervinus, geteilt. Nadelwald. $\mathrm{Da}$ der Pilz unter den Volvariellas nicht zu finden war, habe ich auch die Beschreibungen der Plutei durchgesehen, mit dem gleichen negativen Resultate. Vor allen Dingen ist die Huthaut aller Plutei eine andere.

Agaricus haemorrhoidarius Kalchbr. et Schultz. 18.9. - H. 8 breit, einheitlich braun, mit gleichfarbigen, angedrückten Schuppen. F. mit kleiner Knolle, $10 \times 10$, unten 20 breit. Unterhalb des Ringes braunfleckig. Sp. unter $6 \times 4$.

A. xanthodermus Gen.- 2.10. - Ein kleines Ex. H. 3 breit, kalkweiss, gilbend, klebrig. F. $5 \times 8$, weiss, intensiv gilbend. Sp. $6.5-7 \times 3.5-4$. Schäfferreaktion negativ. Stinkt. Nadelwald. Ich sah bei Wien viel grössere Ex.

$A$. abruptibulbus Peck -8.10 . $-A$. silvicolus nahestehend. Starke flache Knolle (bis 30 breit). Sp. $6-8 \times 4-5$.

A. leucotrichus Moeller - 16.9. - H. bis 10 breit, weiss, gilbend, dicht weissflockig-schuppig. F. bis $10 \times 15$. Basis keulig, unter dem Ring schuppig. Sp. $6 \times 4.5$. Anisgeruch. Nadelwald.

A. augustus Fr. var. albus ss. Moseri. - 11.10.- H. 8 breit, weiss, gelbschuppig. F. $8 \times 15$ (oben) -25 (unten), knollig. Hängender Ring. L. rosa. Sp. $7-9.5 \times 4-6$, gewöhnlich $9 \times 5.5$. Schäfferreaktion positiv. Fl. nicht gilbend. Geruch nach bitteren Mandeln.

Cystoderma carcharias Pers. - 16.9.

Pleurotellus tremulus Schff. - 2.10. - H. bis 2.5 breit, schaufelförmig, grau. F. grau, seitlich, zottig, gut entwickelt. L. grau, dicklich. Sp. ziemlich rund, 5.5-6 im Diam. Im Moose.

Crepidotus variabilis Pers, var. subsphaerosporus Lange 20.9.

Ripartites helomorphus Fr. - 13.9.

R. albido-incarnatus (Britz.) Métr. - 27.9. - H. 2 breit, konvex, fleischfarben, kahl. F. $2 \times 2$, gleichfarbig. L. schwach herablaufend. Sp. etwa $4 \times$ 2.75 .

Tubaria pallidispora Lange ss. Kühn. et Romagn. 10.9 .

T. pellucida Bull. ss. Romagn. -27.9 .

Hebeloma strophosum Fr. - 11.9.

H. mesophaeum Fr. - 19.9 .

H. pumilum Lange - 15.9.

H. truncatum Schff. -24.9 .

Inocybe cervicolor Pers. - 18.9. - H. bis 4 breit, dunkelbraun, auch in der Jugend nicht weiss, faserig schuppig. F. bis $8 \times 6$, unten etwas verdickt, stark bedeckt mit losen, braunen Fasern und unten mit rötlichem Ton. Sp. $11-13 \times$ 7-8. Cyst. auf der Schneide wenig zahlreich, dünnwandig $(40 \times 12)$. Ausserdem haarartige Gebilde (sehr lang und nur 2 breit). Geruch süsslich, nicht sonderlich angenehm. Fl. im F. rötend.

I. pyriodora Pers. var. incarnata Bres. - 15.9.

I. geophylla Sow. var. lilacea - 11.9.

I. dulcamara A. et S. - 19.9. - In allen Teilen, aussen und innen, olivgelb-fuchsig. H. 2-5 breit, schuppig faserig. Sp. $8 \times 5$. Keine schopfigen Cyst.
I. descissa Fr. var. brunneoatra Lange -3.10 .

I. descissa Fr. var. auricoma Batsch -14.9 .

I. sp. - 9.10. - H. 2 breit, kupferbraun, radialfaserig, aufspaltend, spitz gebuckelt. F. $8 \times 3-4$, kahl, gelblich mit schwachem, rötlichem Schimmer. L. grünlicholiv, Schneide durch Cyst. etwas dunkler. Sp. 8.5-9.5(-11) ×6-7 (-8), zugespitzte Eiform, glatt. Cyst. schopfig, $50 \times 10-15$. Geophyllageruch. Die Bestimmung an der Hand der zur Verfügung stehenden Lit. führte zu keinem befriedigendem Resultat. Geht man von der modernen »Flore analytique» von Kühn. et Romagn. aus, so gehört der Pilz sicher zu den Leiosporés cystidiés, groupe 7 . Von den hierher gehörigen Arten kommt am ehesten I. phaeodisca Kühn. (descissa Fr. ss. Bres.) in Frage, eine höchst polymorphe Art, die nicht genügend untersucht ist.

I. abjecta Karst. -2.10 .

I. posterula Britz. ss. Sacc. - 28.9.

I. Langei Heim - 20.9.

I. decipiens Bres. - 17.9. - H. bis 5 breit, stumpf und breit gebuckelt, braunfaserig gestreift, auf blassem Grund. F. kurz und dick, weiss, obenwenn jung - aussen und innen rötlich. Sp. $11 \times 8$ etc. mit zahlreichen, stumpfen Warzen. Cyst. dick (20), schopfig.

Alnicola subconspersa Kühn. (conspersa Pers. ss. Langei, von $A$. luteolofibrillosa Kühn. kaum zu trennen) 25.9. - H. bis 2 breit, ziemlich dunkelbraun, mit gelblichen Flocken besetzt. Schwach hygrophan, durchscheinend gerieft. F. $4 \times 1$, sehr dunkel mit weissgelblichen Flocken. L. zimtfarben, ausgerandet. Sp. $9.5-10 \times 5.5-6$. Unter Erlen.

A. escharoides Fr. - 14.9. - H. bis 2.5 breit, gelbbraun, filzig-schuppig. F. $3 \times 1$, dunkelbraun, weisslich faserig. L. rostgelb. Sp. $10 \times 5.5$. Cyst. mit Schnabel. Bitterlich. Unter Erlen.

A. melinoides (Fr.) Kühn., Rick. (escharoides Konr. et Maubl. non Fr., escharoides Lange) - 22.9. $\mathrm{H}$. bis 1.5 breit, honigfarben, ungerieft. F. bis $5 \times 1$, Farbe des $H$., von unten bräunend. L. ockerlich, buchtig angewachsen. Sp. $10.5 \times 6$ etc., warzig. Bald bitter, bald nicht.

A. amarescens Quel., Kühn. - 14.9. - H. bis 3 breit, spitz oder stumpf kegelig, schwach durchscheinend gerieft, hell kastanienbraun. F. $3 \times 1$, russbraun, feinschuppig. L. blass rötlichbraun. Sp. $10 \times 5.5$, feinwarzig. Cyst. mit Schnabel, bisweilen mit Köpfchen. Bitterlich. Unter Erlen.

A. submelinoides Kühn. - 14.9.

Galerina tibiicystis (Atk.) Kühn. - 24.9.

G. hypnorum Schrank - 25.9

G. nana Petri ss. Kühn. - 1.10. - H. bis 1 breit, halbkugelig, Rand gerieft, ockerbraun, ausbleichend. F. bis $3 \times 1$, gleichfarbig, unten dunkler, weiss-seidig faserig. L. breit angewachsen. Sp. $8-9.5 \times 5-6$, mandelförmig oder eiförmig-spindelig, warzig, dickwandig. Gyst. z. Teil mit Kristallen.

G. marginata Batsch -17.9 .

G. clavata (Vel.) Kühn. - 26.9 - H. unter 1 breit, stumpf kegelig, konvex, dazwischen spitz, lange und stark gerieft, ockergelb. F. $3-4 \times 1$, etwas heller als H. Sp. $11-12 \times 6.5-7$, schwach punktiert. Cystiden: Körper 14, Hals 4 und Kopf 6 breit. Im Moose.

Gymnopilus hybridus Fr. - 13.9. - F. durch seidigen Überzug weisslich, dazwischen ringartig be- 
schleiert. Hyphen der Haut 4-10 im Diam. Cyst. 3 breit, Kopf $4-5$ breit.

Dermocybe cinnamomea L. var. conformis Fr. f. croceifolia Peck - 13.9. - F. gelboliv. L. safranorange. Rettichgeruch.

D. miltina Fr. ss. Quel. et Rick. - 11.9.

D. sanguinea Wulf. -16.9 .

Cortinarius rubicundus (Rea) Pears. (pseudobolaris Maire) - Ein schlechtes Ex. H. 4-5 breit, kupferrot, eingewachsen faserig. F. $10 \times 12$, knollig, faserig, oben weiss, überall rotbraunfleckig. Sp. mandelförmig, 9-10 $95-5.5$. Cyst. haarförmig, 4-5 breit.

G. gentilis Fr. - 18.9.

Hydrocybe saniosa Fr. ss. Langei et Moseri - 28.9.

$H$. saniosa $\mathrm{Fr}$. var. paludosa $\mathrm{R}$. Sch. -28.9 .

H. stemmata Fr. - 7.10. - H. $2-3$ breit, dunkelbraun, trocken kastanienbraun. Rand mit bräunlichen Velumresten besetzt. F. $3-4 \times 3-7$, rötlichbraun, mit braunen Schiippchen. L. olivzimt. Sp. $6-7 \times 3.5$. Fl. bräunlich, mit schwachem rötlichviol. Ton. Nadelwald.

H. parevernia Hry - 4.10.

H. castanea Bull. - 4.10.

H. erythrina Fr. ss. Favre -- 17.9. - Eine der zahlreichen Aberten oder Formen. Sp. $8 \times 5$.

H. bulbosa Sow. - 19.9.

H. subferruginea Batsch var, imbuta Fr. - 11.9.

H. biformis Fr. - 1.10

$H$. armeniaca Schff. - 15.9.

H. pseudoduracina Hry - 3.10. - H. 4 breit, ziegelrot, stark ausblassend. F. $6 \times 5$, weisslich, bräunend, schwach wurzelnd, zäh. Basis weisszottig. L. safranrot. Sp. rund, warzig, 5-5.5 ×4.5-5. Nadelwald.

H. damascena Fr. -20.9 .

H. candelaris Fr. -9.10 .

H. duracina Fr. var. raphanica Moser - 29.9. Rettichgeruch. Bedeutungslose Form.

H. duracina Fr. -3.10 .

H. bovina Fr. ss. Langei - 15.9.

H. glandicolor Fr. -7.10 .

H. helvola Fr. - 27.9 .

H. brunneofulva Fr. -9.10 .

H. alnetorum Vel. - 25.9. - H. 1-3 breit, braun, dunkel gebuckelt. F. bis $8 \times 2$, schwarzbraun, weissgegürtelt. L. rost- resp. dunkelbraun. Sp. $9-9.5 \times 5-5.5$, ei- bis mandelförmig. Riecht etwas wie $H$. hinnulea. Unter Erlen.

H. hemitricha Fr. -22.9 .

H. incisa Pers. - 15.9.

H. striaepilea Favre - 8.10.-H. 2 breit, dunkelbraun, trocken mehr rostfarben, kegelig-gebuckelt, gewölbt, stark gerieft. F. $4 \times 3$, braun mit schmutzig-weisslicher Ringzone. L. gedrängt, zimtbraun. Sp. 8-9.5 $\times 5-5.5$, mandelförmig.

H. flexipes Pers. -23.9 .

H. paleacea Weinm. - 19.9.

H. rigida Scop. ss. OvS. - 13.9. - Sehr fest. H. bis 4 breit, gebuckelt, kastanienbraun, trocken fuchsig. Nur Rand mit weissen Velumresten. F. $5 \times 6$, braun, mit weisser Gürtelzone. L. Farbe des H., schwach ausgebuchtet angewachsen. Sp. $6 \times 3.5$, ziemlich glatt und hell. Entspricht Mosers Pilz, hat aber keinen Pelargoniumgeruch.

H. Adalberti Fav. (pateiformis Fr. ss. Rick.) - 26.9. H. bis 5 breit, dunkelkastanienbraun. Mitte beinahe schwarz. Trocken kastanienbraun. Rand schwach weissfaserig. $F$. bis $5 \times 8$, bräunlich, weisslich überfasert, mit schwacher Ringzone. L. auffallend dunkelsafran. Sp. sehr klein, die grössten $7 \times 4$, gewöhnlich $6 \times 3.5$.

H. rubricosa Fr. - 15.9 .

H. uracea Fr. ss. Langei -5.10 .

H. scandens Fr. -17.9 .

H. obtusa Fr. - 11.9 .

H. acuta Fr. ss. Favre -10.9 . - Sp. 7.5-9 $\times 5-6$, glat.t. Zahlreiche haarförmige, sehr lange Cyst. auf der L. schneide.

H. acuta Fr. ss. Hry - 30.9. - Sp. 9-12 $\times 5-6$ mandelförmig, warzig. Cyst. keulig, etwa 12 breit. NB. Am 1.10. fand ich eine dritte Form mit $8 \times 5$, ziemlich glatten Sp. und keulenförmigen Gyst. Die Problematik dieser Sammelart wird dadurch nur noch weiter unterstrichen.

H. acuta Fr. var. striatula Hry (obtusa ss. Langei) 2.10. - H. bis 3 breit, bis zur Mitte gerieft, gelb-, bis orangebraun, flach gebuckelt. F. $3-6 \times$ $4-7$, blass, unten meist zugespitzt. Sp. mandelförmig, meist $8.5 \times 5$. Durch Phenol rot. Übergangsform zu $H$. acuta.

Myxacium elatior Fr. -21.9 .

M. causticum Fr. - 29.9. - Nur ein Ex. H. 3 breit, lebhaft gelb, sehr schleimig. F. $8 \times 6$, unten zugespitzt, weisslich. L. ocker. Sp. $7.5 \times 4$. Bitter.

Phlegmacium calochroum Pers. - 28.9. - H. bis 6 breit, löwengelb, schmierig. Dunkelrote Laugenreaktion auf dem H. F. bis $5 \times 15$, weisslich. Gerandete, niedergedrückte Knolle. L. rosa-violett. Sp. $10.5-11.5 \times 5.5-6$, mandelförmig, warzig. Fl. weiss. Nadelwald.

P. ferrugineum Fr. ss. Rick. et Moseri - 14.9. - H 6 breit, rötlichbraun, niedergedrückt. Rand lange eingerollt. F. $5 \times 10$. Keule rundlich, $20 \mathrm{im}$ Diam. L. rostbraun. Sp. $8 \times 5$.

P. claricolor Fr. ss. Konr. et Maubl. - 30.9. - Ohne bläuliche Töne. Ohne unangenehmen Geruch. Nadelwald.

P. subtriumphans Hry -27.9 .

P. decolorans Pers. ss. Cke -29.9 .

P. subpurpurascens (Fr.) Rick. ss. Kauffm. et Moseri - 20.9. - Vielleicht nicht identisch mit P. subpurpurascens Batsch. Siehe Kap. 4. H. bis 6 breit, kastanienbraun. F, bis $10 \times 10-15$, ziemlich dunkelviolettlich, Knolle verschwindend. L. braun mit viol. Ton. Sp. mandelförmig, $8-9 \times 4.5$ -5. F. und L. (diese weniger) an Druckstellen purpurnfleckend. Fl. bald ganz unveränderlich, bald im F. deutlich purpurn anlaufend. Die Veränderlichkeit resp. Unveränderlichkeit des Fl. ist als Kriterium zur Abgrenzug der Arten purpurascens und subpurpurascens wenig zuverlässig.

P. odorifer Britz. - 11.9. - H. 8 breit, ziegelrot, schmierig. Rand etwas grünlich. F. $7 \times 10$ (oben) -25 (unten), intensiv gelbgrün. L. gleichfarbig, stark ausgebuchtet und breit. Sp. 10-11 $\mathrm{x}$ 5.5-6, mandelförmig, warzig. Fl. gelbgrün, durch KOH gelb, dann rötend. Süsser Geruch. Nadelwald. Steht $P$. orichalceum mindestens sehr nahe.

P. orichalceum Batsch -28.9 .

P. flavescentium Hry - 29.9. - H. 5 breit, gelbgrün bis gelb, Mitte kupferfarben, also etwa die normale Färbung von Naem. sublateritium F. $10 \times 8$, gerandete Knolle. F. und L. grünlichgelb. Sp. $11-12 \times 6.5-7$. Fl. weisslich. Laugenreaktion blutrot. Geruchlos. Steht $P$. orichalceum und $P$. cedretorum Maire sehr nahe. 
P. sulphureum Lange - 21.9. - H. 5 breit, olivgelb, grünlich am Rande und olivbraun in der Mitte. F. $8 \times 30$, grüngelb, L. gleichfarbig. Sp. $9-10 \times 5.5-6$, mandelförmig. Mischwald.

P. fulgens A. et S. ss. Fr. - 21.9 - Nur ein schlechtes Ex. H. 6 breit, löwengelbbraun, sehr klebrig. F. $6 \times 10-25$, gelblich, bräunlich faserig gestreift. Deprimierte, gerandete Knolle. L. gelb mit sehr schwachem Olivton. Sp. $11-12 \times 6-7$, sehr dunkel, warzig. Fl. weisslich, im F. gelblich. Nadelwald.

P. elegantior Fr. ss. Moseri - 4.10. - H. bis 12 breit, durchweg rostbraun. F. bis $8 \times 25$, schmutziggelblich. Knolle bis 40 breit. L. gelblich, bei ausgewachsenen Ex. beinahe oliv. Sp. $13-14 \times 8$, mandel- bis eiförmig. Fl. gelblich, in der Knolle rhabarberfarben. Nadelwald.

P. infractum Pers. - 16.9 .

Conocybe tenera Sxhff. var. semiglobata Kühn. - 12.9 . Bolbitius titubans Bull. - 22.9.

Pholiota heteroclita Fr. - 22.9.

P. squarrosa Müll. - 10.10.

P. flammans Fr. - 18.9 .

P. subsquarrosa Fr. $-22.9-$ H. 6 breit, braungelb braunschuppig, sehr klebrig. F. $6 \times 10$, trocken braungelb, beinahe sparrig-braunschuppig. L. blass, ausgebuchtet. Sp. $6-6.5 \times 4-4.5$, Cyst. mit unregelmässiger Flaschenform. Auf Birke.

$P$. aurivela Batsch - 14.9. - $\mathrm{H}$. bis 10 breit, rostgelb, mit dunklen, angedrückten Schuppen. F. kurz, gleichfarbig, bisweilen beinahe sparrig geschuppt. L. gelb, später olivbraun. Sp. c. $8 \times 5$. Auf lebender Erle.

P. lenta Pers. -1.10 . - H. bis 7 breit, weisslich mit im Schleim schwimmenden weissen Flöckchen. F. bis $10 \times 10$, weisslich, mit bräunlicher Basis, flockig-schuppig. L. blass. Sp. $6-7 \times 4$.

P. gummosa Lasch - 25.9. - H. bis 4 breit, weisslich, gilbend, angedrückt weiss-schuppig. F. bis $10 \times 10$, gelb, unten rostfarben, schuppig. L. gelblich. Sp. $6-7.5 \times 4-4.5$, oval. Cyst. haarförmig, 4-6 (oben) im Diam. Fl. weissgelb, unten im F. rostfarben. Milder Geschmack. Ohne Geruch. An und neben Laubholzstrünken, auch auf Wurzeln. Forma ochroclora grünlich.

P. astragalina $\mathrm{Fr} . \quad-12.9$.

P. flavida Schff. - 19.9. - Schwefelgelb. Büschelig auf Nadelholzstämmen. Sp. $7-8.5 \times 4-5.5$.

Stropharia aeruginosa Curt. - 21.9.

S. albocyanea (Desm.) Fr. - 24.9. - Wie S. acruginosa, nur kleiner, mit blasseren Farben und weissem F. Kaum sebständige Art. Übergänge zu häufig.

Panaeolus fimicola Fr. var. ater Lange - 20.9 .

Psathyrella microrhiza Lasch f. polycystis Romagn. 27.9. - H. bis 2 breit, schmutzigbraun, trocken beinahe rosa (fleischfarben). Rand gerieft. F. bis $8 \times 2$, weisslich, wurzelnd. L. mit rötlicher Schnejde. Sp. $11 \times 7$, dunkel, aber nicht schwarz. Gyst. zahlreich und schmal $(7-11$ breit), mit bisweilen etwas verbreiteter Spitze. Weideland.

P. spadicea Schff. -11.9 .

$P$. obtusata Fr. -22.9 .

Coprinus lagopus Fr. -23.9

C. plicatilis Curt. - 18.9 .

C. auricomus Pat. (hemerobius Fr.) - 12.9. - H. einige $\mathrm{cm}$ breit, grauend. Scheibe dauernd ocker. Hier und da lange Haare auf der Huthaut. F. weiss, nackt, bisweilen sehr lang, oben verbreitet, aber ohne richtiges Scheibchen. Sp. etwa $12 \times 7$, durchsichtig, aber sehr dunkelbraun. Sieht C. plicatilis sehr ähnlich.

Russula ochroleuca Pers. - 16.9.

$R$. xerampelina Schff. var. erythropus (Pelt.) M. etZ.13.9.

R. nitida Pers. ss. Pears. -26.9 .

R. alutacea Fr. ss. Melz. Zv. -26.9.

R. emetica Schff. var. crenulata Burl. - 30.9. - Häufig ausgeblichen. L. schwach gesägt. Warzen der Sp. ziemlich niedrig.

R. emetica Schff. var. silvestris (Sing.), f. pithyophila Romagn. - 13.9.

$R$. violacea Quel. -20.9 .

$R$. atropurpurea Krombh. - 29.9. - H. 8 breit, violettpurpurn. Nur Rand abzichbar. F. bis $6 \times 30$, weiss, fest. Sp. B, Orn. 4, Grösse $8 \times 7$. Fl. fest,' scharf. Obstgeruch.

Lactarius vietus Fr. - 11.9.

L. pyrogalus Bull. - 16.9. - Milch weiss, scharf, zu Quark werdend. H. bis 7 breit, braungrau bis ledergelb, undeutlich gezont, feucht. F. kahl, blass, $4-5 \times 12$. L. sehr dünn, ocker, häufig mit verhärteten, weissgelblichen Tropfen besetzt. Sp. $7 \times 6$, crème-gelblich. Unter Hasel.

L. lilacinus Lasch -11.9 .

L. mitissimus Fr. -10.9 .

L. subdulcis (Bull.) Fr. ss. restr. Lange - 30.9.

Cantharellus infundibuliformis Scop. - 18.9.

C. albidus Fr. - 23.9.

Scleroderma verrucosum Bull. - 11.9.

S. bovista Fr. - 13.9. - Beinahe glatt, gelblich, rundlich. Fruchtmasse wird olivbraun. Sp. grobwarzig, etwa $10-13$ im Diam.

Calvatia saccata Vahl - 17.9.

Clavaria flaccida Fr. - 20.9 .

C. cristata Holmsk. -5.10 .

C. lappa Karst. -10.9 .

C. muscoides L. - 8.10.

C. fastigiata L. (muscoides L. var. pratensis Cott. et Wakef.) - 13.9. - Sehr klein, sehr verästelt, sonst wie C. muscoides L. Sp. 3-4 im Diam.

C. aurantia Pers. - 15.9. - Einzelkeulen, einige $\mathrm{cm}$ lang und einige $\mathrm{mm}$ breit, orange. $\mathrm{Sp}$. etwa $5 \times 4$, eintropfig. Basis verschmälert, bisweilen mit nur angedeutetem Stielchen. Myzel weiss. Rasig, auf Weideland.

C. argillacea Pers. - 26.9. - Blassgelb mit ausgesprochen gelbem Stiel, $3 \mathrm{~cm}$ lang und einige $\mathrm{mm}$ dick, keulenförmig, cylindrisch oder bisweilen oben verbreitet und gabelig geteilt. Sp. $8-9 \times$ $4.5-5$, farblos, glatt. Nasses Ödland.

C. ligula Schff. - 19.9.

Merulius tremellosus Schrad. - 17.9.

Dryodon diversidens Fr. - 18.9.

Phylacteria palmata Scop. - 30.9.

P. terrestris Ehrh. - 15.9. 
Trametes rubescens A. et S. - 15.9. - Nach Overholts eine Form von Daedalea confragosa Bolt. H. 5 breit, halbkreis- oder nierenförmig, zart filzig, schwach gezont, weisslich, dann rötlich. Ohne F. Auf toten Laubhölzern. Steht Lenzites tricolor sehr nahe. Das Exs. stellt eine Übergangsform zu $L$. tricolor dar.

Ganoderma sp. - 21.9. - Entspricht äusserlich dem in Kap. 5 sub Ganoderma sp. beschriebenen Pilz. Unterschiede: R. 0.4 bis $0.8 \mathrm{~mm}$ lang. P. $0.2-0.25$ im Diam. Sp. braun, $8-9 \times 5.5$, oben abgestumpft, rauh. Vermutlich handelt es sich beim Funde auf Åland um ältere Ex. desselben Pilzes. Ein Fund vom 9.10. zeigte $9-10.5 \times 5.5$ $\mathrm{Sp}$.
Xanthochrous cinnamomeus Jacq. - 4.10. - Zusammenfliessende bis 4 breite, zimtfarbene, braungezonte, sammetige Hütchen. F. kurz und dünn, zimtfarben, sammetig. R. zimtbraun, kurz (2 $\mathrm{mm}$ ), höchst unregelmässig, bisweilen labyrinthisch verbunden (daedaleaartig). Fl, zimt, dünn (1 mm), lederzäh. Auf den total verwesten Resten eines unbestimmbaren Stammes. Exs. im Forstwissenschaftlichen Institut von Helsinki.

$$
\text { ASCOMTCETES. }
$$

Gyromitra infula Schff. - 29.9.

Cudonia circinans Pers. - 17.9 .

Macropodia macropus Pers. - 3.10 . 